\title{
The Interaction of Lighting, Heating and Cooling Systems in Buildings
}

Stephen J. Treado

John W. Bean

Building and Fire Research Laboratory

Gaithersburg, Maryland 20899

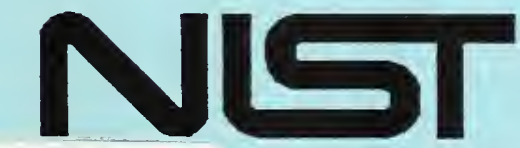

$\begin{array}{ll}\mathrm{QC} & \text { A Department of Commerce } \\ 100 & \text { Administration } \\ .456 & \\ 4701 & \\ 1992 & \\ \text { C. } 2 & \end{array}$




\section{The Interaction of Lighting, Heating and Cooling Systems in Buildings}

Stephen J. Treado

John W. Bean

\section{March 1992}

Building and Fire Research Laboratory

National Institute of Standards and Technology

Gaithersburg, MD 20899

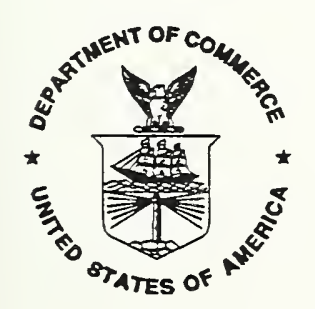

U.S. Department of Commerce

Barbara Hackman Franklin, Secretary

Technology Administration

Robert M. White, Under Secretary for Technology

National Institute of Standards and Technology

John W. Lyons, Director 


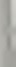




\begin{abstract}
The interaction of building lighting and HVAC systems, and the effects on cooling load and lighting system performance, are being evaluated using a full-scale test facility at the National Institute of Standards and Technology. The results from a number of test configurations are described, including measurements of lighting system efficiency and cooling load due to lighting. The effect of lighting and HVAC system design and operation on performance is evaluated. Design considerations are discussed.
\end{abstract}

KEYWORDS: airflow, cooling, energy, lighting, load calculation, ventilation 


\section{TABLE OF CONTENTS}

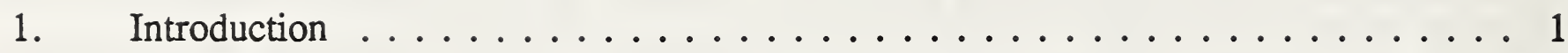

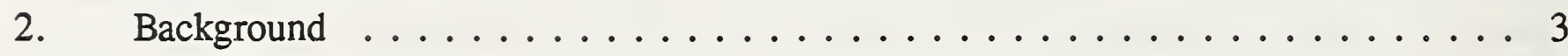

3. Test Facility $\ldots \ldots \ldots \ldots \ldots \ldots \ldots \ldots \ldots \ldots \ldots \ldots$

4. Results ............................ 7

4.1 Lighting System Performance and Efficiency $\ldots \ldots \ldots \ldots \ldots . \ldots 9$

4.1.1 Four Lamp Acrylic Lens Luminaire . . . . . . . . . . . . 10

4.1.2 Four Lamp Parabolic Diffuser Luminaire . . . . . . . . . . 12

4.1.3 Two Lamp Acrylic Lens Luminaire . . . . . . . . . . . . . 13

4.2 Lighting Cooling Load Regression Analysis . . . . . . . . . . . 14

4.2.1 Four Lamp Parabolic Luminaire . . . . . . . . . . . . . . . 15

4.2.2 Four Lamp Acrylic Lens Luminaire . . . . . . . . . . . 18

4.2.3 Two Lamp Acrylic Lens Luminaire . . . . . . . . . . . . . . 19

4.3 Cooling Load Profiles . . . . . . . . . . . . . . . . . . . 19

4.3.1 Four Lamp Parabolic Luminaire . . . . . . . . . . . . . 20

4.3.2 Four Lamp Acrylic Luminaire . . . . . . . . . . . . 20

4.3.3 Two Lamp Acrylic Luminaire . . . . . . . . . . . . 20

4.4 Peak Cooling Loads due to Lighting . . . . . . . . . . . . . 20

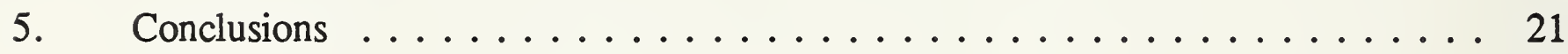

6. References ........................... 22 


\section{LIST OF TABLES}

Table $1 \quad$ Basic Test Configurations $\ldots \ldots \ldots \ldots \ldots \ldots \ldots \ldots$

Table 2 Lamp Temperature Elevations . . . . . . . . . . . . . . 10

Table 3 Airflow Rates for Testing . . . . . . . . . . . . . . . . . 14

Table $4 \quad$ Measured Cooling Load Results . . . . . . . . . . . . . . . . . . 16 


\section{LIST OF FIGURES}

Figure 1. Energy distribution fractions from a typical luminaire . . . . . . . . . 23

Figure 2. Cooling load profile with cyclic lighting . . . . . . . . . . . . 24

Figure 3. Temperature dependence of fluorescent lamps $\ldots \ldots \ldots \ldots \ldots$

Figure 4 . Cut-away schematic view of lighting test facility $\ldots \ldots \ldots \ldots \ldots$

Figure 5. Typical transient test of cooling due to lighting $\ldots \ldots \ldots \ldots \ldots$

Figure 6. Measured cooling load, lighting power and airflow rate

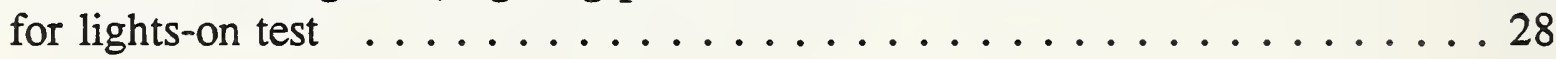

Figure 7. Measured room and plenum air temperatures for lights-on test . . . . . . . . . . . . . . . . . . . . 29

Figure 8. Measured floor and ceiling surface temperatures for lights-on test . . . . . . . . . . . . . . . . 30

Figure 9. Measured room and plenum wall temperatures for lights-on test . . . . . . . . . . . . . . . 31

Figure 10. Measured lamp temperatures for lights-on test $\ldots \ldots \ldots \ldots$. . . . . 32

Figure 11. Double exponential curve fit to measured cooling load due to lighting . . . . . . . . . . . . . . . 33

Figure 12. Minimum lamp wall temperatures for the four lamp acrylic lens luminaire . . . . . . . . . . . . . . . . . 34

Figure 13. Elevation of minimum lamp wall temperature above room air temperature for the four lamp acrylic lens luminaire . . . . . . . . . . . . . . . . . 35

Figure 14. Lighting power, light output and luminous efficacy for the four lamp acrylic lens luminaire with ceiling grill return

Figure 15. Lighting power, light output and luminous efficacy for the four lamp acrylic lens luminaire with lamp compartment return . . . . . . . . . . . . . . . . . 37 
Figure 16. Luminous efficacy for the four lamp acrylic lens luminaire with ceiling grill or lamp compartment

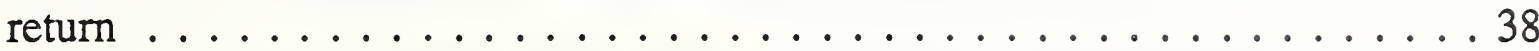

Figure 17. Minimum lamp wall temperatures for the four lamp parabolic diffuser luminaire . . . . . . . . . . . . . . . . . . . . 39

Figure 18. Elevation of minimum lamp wall temperature above room air temperature for the four lamp parabolic diffuser luminaire . . . . . . . . . . . . . . . . . . . . 40

Figure 19. Lighting power, light output and luminous efficacy for the four lamp parabolic diffuser luminaire with ceiling grill return

Figure 20. Lighting power, light output and luminous efficacy with side slot return

Figure 21. Lighting power, light output and luminous efficacy for the four lamp parabolic diffuser luminaire with lamp compartment return . . . . . . . . . . . . . . 43

Figure 22. Luminous efficacy for the four lamp parabolic diffuser luminaire with ceiling grill, side slots or lamp compartment return

Figure 23. Minimum lamp wall temperature for four two-lamp acrylic lens luminaires with ceiling grill or lamp compartment return

Figure 24. Elevation of minimum lamp wall temperature above room air temperature for four two-lamp acrylic lens luminaires

Figure 25. Lighting power, light output, and luminous efficacy for four two-lamp acrylic lens luminaires with ceiling grill return

Figure 26. Lighting power, light output, and luminous efficacy for four two-lamp acrylic lens luminaires with lamp compartment return . . . . . 48 48

Figure 27. Luminous efficacy for four two-lamp acrylic lens luminaires with ceiling grill and lamp compartment return . . . . . . . . . . . . 49

Figure 28. Minimum lamp wall temperature for three two-lamp acrylic lens luminaires with ceiling grill or lamp compartment return . . . . . . . 50 
Figure 29. Elevation of minimum lamp wall temperature above room air temperature for three two-lamp acrylic lens luminaires

Figure 30. Lighting power, light output, and luminous efficacy for three two-lamp acrylic lens luminaires with ceiling grill return $\ldots \ldots \ldots 52$

Figure 31. Lighting power, light output, and luminous efficacy for three two-lamp acrylic lens luminaires with lamp compartment return . . . . 53

Figure 32. Luminous efficacy for three two-lamp acrylic lens luminaires with ceiling grill and lamp compartment return . . . . . . . . . . 54

Figure 33. Minimum lamp wall temperature for three two 34-watt lamp acrylic lens luminaires with ceiling grill or lamp compartment return . . . . 55

Figure 34. Elevation of minimum lamp wall temperature above room air temperature for three two 34 -watt lamp acrylic lens luminaires . . . . . . 5 56

Figure 35. Lighting power, light output, and luminous efficacy for three two 34 -watt lamp acrylic lens luminaires with ceiling grill return . . . . . 57

Figure 36. Lighting power, light output, and luminous efficacy for three two 34-watt lamp acrylic lens luminaires with lamp compartment return . . . . . . . . . . . . . . 58

Figure 37. Luminous efficacy for three two 34-watt lamp acrylic lens luminaires with ceiling grill and lamp compartment return

Figure 38. Cooling load regression for the four lamp parabolic diffuser luminaires with lamp compartment return . . . . . . . . . . . 60

Figure 39. Cooling load regression for the four lamp parabolic diffuser luminaires with ceiling grill return

Figure 40. Cooling load regression for the four lamp parabolic diffuser luminaires with side slot return

Figure 41. Cooling load regression for the four lamp parabolic diffuser luminaires with lamp compartment return, carpet

Figure 42. Cooling load regression for the four lamp parabolic diffuser luminaires with side slot return, carpet 
Figure 43. Cooling load regression for the four lamp parabolic diffuser luminaires, $120 \mathrm{cfm}$ airflow, lamp compartment and ceiling grill return . . . . . . . . . . . . . . . . . . . 65

Figure 44. Cooling load regression for the four lamp parabolic diffuser luminaires, $160 \mathrm{cfm}$ airflow, lamp compartment and ceiling grill return . . . . . . . . . . . . . . . . . . . 66

Figure 45. Cooling load regression for the four lamp parabolic diffuser luminaires, $200 \mathrm{cfm}$ airflow, lamp compartment and ceiling grill return . . . . . . . . . . . . . . . . . . . 67

Figure 46. Cooling load regression for the four lamp parabolic diffuser luminaires, $120 \mathrm{cfm}$ airflow, lamp compartment return, with

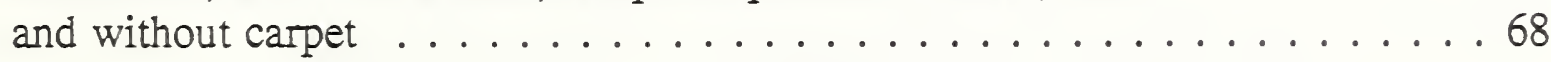

Figure 47. Cooling load regression for the four lamp parabolic diffuser luminaires, $160 \mathrm{cfm}$ airflow, lamp compartment return, with and without carpet . . . . . . . . . . . . . . . . . 6 69

Figure 48. Cooling load regression for the four lamp parabolic diffuser luminaires, $200 \mathrm{cfm}$ airflow, lamp compartment retum, with and without carpet . . . . . . . . . . . . . . 70

Figure 49. Cooling load regression for the four lamp acrylic lens luminaires, low air flow ( $96 \mathrm{cfm})$, lamp compartment return, carpet . . . . . . . . . . . . . . . 71

Figure 50. Cooling load regression for the four lamp acrylic lens luminaires, 120 and $200 \mathrm{cfm}$ air flow, ceiling grill return, carpet

Figure 51. Cooling load regression for the four lamp acrylic lens luminaires, $120 \mathrm{cfm}$ air flow, ceiling grill and lamp compartment return, carpet . . . . . . . . . . . . . . . 73

Figure 52. Cooling load regression for the four lamp acrylic lens and parabolic diffuser luminaires, $120 \mathrm{cfm}$ air flow, lamp compartment return . . . . . . . . . . . . . . . . . . 74

Figure 53. Cooling load regression for the four lamp acrylic lens and parabolic diffuser luminaires, $200 \mathrm{cfm}$ air flow, ceiling grill return 
Figure 54. Cooling load regression for four two-lamp acrylic lens luminaires, ceiling grill return, carpet, effect of air flow ceiling grill return $\ldots \ldots \ldots \ldots \ldots \ldots$

Figure 55. Cooling load regression for four two-lamp acrylic lens luminaires, ceiling grill return, carpet, effect of air flow

Figure 56. Cooling load regression for four two-lamp acrylic lens luminaires, lamp compartment return, carpet, effect

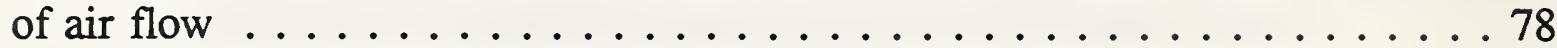

Figure 57. Cooling load regression for four two-lamp acrylic lens luminaires, ceiling grill return, carpet, unfurnished

Figure 58. Cooling load regression for four two-lamp acrylic lens luminaires, ceiling grill return, carpet, furnished

Figure 59. Cooling load regression for four two-lamp acrylic lens luminaires, sealed ceiling grill return, carpet, unfurnished

Figure 60. Cooling load regression for four two-lamp acrylic lens luminaires, ceiling grill return, carpet, effect of furnishings

Figure 61. Cooling load regression for four two-lamp acrylic lens luminaires, ceiling grill return, carpet, effect of furnishings

Figure 62. Cooling load profile for the four lamp parabolic diffuser luminaires with lamp compartment return

Figure 63. Cooling load profile for the four lamp parabolic diffuser luminaires with ceiling grill return

Figure 64. Cooling load profile for the four lamp parabolic diffuser luminaires with side slot return

Figure 65. Cooling load profile for the four lamp parabolic diffuser luminaires with lamp compartment return, carpet

Figure 66. Cooling load profile for the four lamp parabolic diffuser luminaires with side slot return, carpet

Figure 67. Cooling load profile for four four-lamp acrylic lens luminaires with lamp compartment return, carpet 
Figure 68. Cooling load profile for four four-lamp acrylic lens luminaires

with ceiling grill return, carpet . . . . . . . . . . . 90

Figure 69. Cooling load profile for four two-lamp acrylic lens luminaires

with ceiling grill return, carpet . . . . . . . . . . . . 91

Figure 70. Cooling load profile for four two-lamp acrylic lens luminaires

with lamp compartment return, carpet . . . . . . . . . . . . 92

Figure 71. Cooling load profile for four two-lamp acrylic lens luminaires

with ceiling grill return, carpet . . . . . . . . . . . . 93

Figure 72. Cooling load profile for four two-lamp acrylic lens luminaires

with ceiling grill return, carpet, furnished . . . . . . . . . . . . 94

Figure 73. Cooling load profile for four two-lamp acrylic lens luminaires

with sealed ceiling grill return, carpet . . . . . . . . . . . 95

Figure 74. Peak cooling load factor for four fixture acrylic lens luminaires

with lamp compartment return, carpet . . . . . . . . . . . 96

Figure 75. Peak cooling load factor for four fixture acrylic lens luminaires

with ceiling grill return, carpet . . . . . . . . . . . . 97

Figure 76. Peak cooling load factor for four four-lamp parabolic diffuser luminaires with ceiling grill return $\ldots \ldots \ldots \ldots . \ldots . \ldots 9$

Figure 77. Peak cooling load factor for four four-lamp parabolic diffuser luminaires with lamp compartment return . . . . . . . . . . . . 999

Figure 78. Peak cooling load factor for four four-lamp parabolic diffuser luminaires with side slot return . . . . . . . . . . . . . . . . . . 100

Figure 79. Peak cooling load factor for four four-lamp parabolic diffuser luminaires with lamp compartment return, carpet . . . . . . . . . 101

Figure 80. Peak cooling load factor for four four-lamp parabolic diffuser luminaires with side slot return, carpet . . . . . . . . . . . . . . . 102

Figure 81. Peak cooling load factor for four two-lamp acrylic lens luminaires with ceiling grill return, carpet . . . . . . . . . . . 103

Figure 82. Peak cooling load factor for four two-lamp acrylic lens luminaires with ceiling grill return, carpet, furnished 



\section{Introduction}

Energy requirements for commercial buildings are primarily attributable to lighting, heating, cooling and ventilation. As energy resources continue to diminish, and energy costs and demands continue to rise, building designers, owners and occupants look for means to improve building energy efficiency and control peak thermal loads. The emphasis on improved thermal performance has led to the development of better windows and building envelope components, more efficient lighting and HVAC equipment and intelligent, integrated control systems.

In the midst of these advances in building technology, building designers are faced with the task of selecting the appropriate building components and equipment in order to achieve optimum total performance. The total building performance, as distinct from individual component performance, includes the integrated effects of the entire building with its lighting, HVAC and control systems interacting in a realistic manner, both among themselves and with the building occupants.

The interactions among building sub-systems are important for two reasons. First, no building component exists in a vacuum. It is always part of a larger framework and subject to variable conditions and demands. Unless the interactions between different building components are considered, design decisions may just be plain wrong. Second, optimum system performance cannot be achieved without evaluating the performance of the total system because of the interdependence of performance characteristics. Once the individual component performance characteristics have been maximized, further improvement can only be obtained by optimizing total system performance.

The need to consider the interactions between building systems and components puts extreme demands on design procedures and tools. Traditional design methods have been based on separately evaluating each component with little regard for other building systems. Thus, the lighting system would be designed independently from the HVAC system, without regard for optimizing the combined system performance.

This research project is intended to provide technical information to enable the interactions between the lighting and HVAC system to be understood and utilized to provide for efficient combined system performance. In this manner, both lighting system efficiency and cooling load control can be achieved.

Lighting in commercial buildings is the single largest user of electric energy, typically ranging from 25 to 50 percent of total building electrical energy requirements. In 1980, 2.8 $\left(10^{15}\right)$ Btu $\left(2.95\left(10^{15}\right) \mathrm{kJ}\right)$ of energy was used for lighting for a resulting total cost of over 40 billion dollars annually [1]. An overall increase in lighting efficiency of one percent would produce a savings of 400 million dollars a year. 
The performance of the dominant commercial light source, the fluorescent lamp, is strongly dependent on thermal conditions. Both lamp light output and power consumption vary with minimum lamp wall temperature as much as 20 percent under typically encountered conditions. Proper control of room thermal conditions can ensure that the lamps are operating at their most efficient level.

In addition to the electrical energy purchased and used for lighting itself, heat dissipated from the lighting system adds to the building cooling load in summer, and decreases the heating requirements in winter. Controlling peak cooling loads in summer is of particular interest to electric utilities which have to reduce summer peaks to control peak electric power demands on hot summer afternoons. Due to finite electric power generation resources, steady electric power demands make most efficient use of electric power generation facilities. The capital cost of expansion of generating capacity has, in turn, led to greater costs to the user in the form of demand charges and ratchet clauses. A demand charge usually takes the form of a higher unit cost for electrical power during periods of heavy system-wide usage. Demand charges frequently are assessed from late morning through early evening.

A ratchet clause ties the unit cost for electric power for the entire year to the maximum electric power demand over a specified interval. The interval might be several hours or longer during periods of heavy usage. With this sort of clause, even a single day of excessive electrical demand could result in significant increases in the total annual cost of lighting energy. Higher peak cooling loads also require larger equipment sizes to maintain comfort conditions, resulting in higher first costs.

This report is part of a research effort to determine energy transfer from lighting systems to building spaces and to evaluate the performance of lighting and HVAC equipment as influenced by typical operating conditions and equipment configurations. The program is cofunded by the U.S. Department of Energy and the Electric Power Research Institute. The objective of the research effort is to develop procedures to promote the design of efficient lighting and HVAC systems, leading to energy and cost savings. A combination of detailed full scale measurements of lighting and HVAC system performance and related computer simulations forms the basis of the approach to these issues. More detailed information on the experimental plan and test facility design are contained in [2] and [3].

A full-scale test facility was constructed to simulate an office space. The test facility was extensively instrumented to allow the measurement of lighting levels, electrical power consumption, heat transfer and temperatures. Various lighting and HVAC system designs have been tested, along with other room-related parameters. The parameters which have been varied include:

- luminaire type

- lamp type

- number of lamps per luminaire

- room air temperature 
- airflow rate, constant and variable

- return airflow path

- carpet

- furnishings

- internal electric loads

A detailed computer model of the test facility has been developed and is being used to extend the measurement results [3]. This model will also form the basis of a sub-routine which can be incorporated into larger building energy analysis computer program.

This report describes the results from an analysis of a series of measurements of lighting system efficiency and cooling load due to lighting. The effects of lighting and HVAC design and operation on lighting system power input, light output and efficiency are presented. The effect of lighting and HVAC design on cooling load due to lighting, particularly peak cooling load, is examined. Correlations are described and presented to aid in the design of efficient lighting and HVAC systems.

\section{Background}

There are two major issues associated with the interaction of lighting and HVAC systems in buildings. One issue is the efficiency of the lighting system. Energy for lighting and the number of luminaires required to provide the desired light levels will be minimized if the luminaires are operated at their efficiency temperature. The total heat gain to the building space from the luminaire would also be minimized at this condition, meaning minimum cooling loads from lighting. Luminaire temperatures are determined by the total room thermal environment, including air and surface temperatures, airflow rates, and supply and return air configurations.

Figure 1 shows the energy distribution components from a lighting fixture, or luminaire. All of the electrical energy input to the luminaire is dissipated into the building space as visible light, convection, conduction or thermal radiation. Some of the energy goes into the room and some into the plenum if there is one present. The net effect of all of these modes of heat transfer determine the luminaire heat balance and equilibrium temperature.

The second issue relates to the peak cooling loads due to lighting. When a lighting system is switched on, all of the electrical power input does not show up as cooling load immediately, because of heat storage in building components, plenum air (if any) and the luminaires themselves. Of course, once the lights are turned off, the stored heat will be dissipated to the room air and eventually appear as cooling load. Thus, peak cooling loads due to lighting can be controlled by channeling some of the energy dissipated by the luminaire into components such as floor and ceiling slabs, walls and furnishings, thereby redistributing the cooling load due to lighting over a longer period of time. 
Figure 2 shows a typical profile of cooling load due only to lighting for cyclic daily operation of the lights. The cooling load is plotted relative to the lighting power. Maximum cooling load due to lighting occurs just before the lights are switched off. The difference between the peak cooling load due to lighting and the lighting input power is the peak load reduction.

Maximum light output and power consumption occur when the lamp cold spot is approximately $104^{\circ} \mathrm{F}\left(40^{\circ} \mathrm{C}\right)$, although they do not necessarily occur at exactly the same temperature, as shown in figure 3. Maximum lamp luminous efficacy also occurs near $104^{\circ} \mathrm{F}\left(40^{\circ} \mathrm{C}\right)$ usually coincident with the point of maximum light output. The exact temperature dependence of different lamps varies somewhat, but all display the same general behavior as shown in figure 3 [4].

Other factors also influence fluorescent lamp performance including lamp length and diameter, lamp loading and argon pressure. However, these other factors are usually fixed for a particular lamp installation, and not dependent on thermal conditions. On the other hand, the thermal conditions to which a fluorescent luminaire, that is the lamp and fixture combination, is exposed can significantly alter lamp temperature, and thus, lamp performance. These thermal conditions include air temperatures, airflow conditions near the luminaire and thermal radiation exchange with surrounding surfaces. It is important to note that the thermal environment is somewhat under the control of the building designer, allowing it to be tailored to promote efficient luminaire operating conditions.

\section{Test Facility}

The test facility is constructed on a slab $30 \mathrm{ft} .6$ in. $(9.30 \mathrm{~m})$ by $21 \mathrm{ft} .4$ in. $(6.50 \mathrm{~m})$ within the large NIST environmental chamber. The facility is divided into two sections, a large insulated shell enclosing the test room area, and a smaller attached control room for housing instrumentation. The overall height is $20 \mathrm{ft} .101 / 2 \mathrm{in}$. $(6.36 \mathrm{~m})$, while the control room ceiling height is $13 \mathrm{ft} .21 / 2$ in. $(4.03 \mathrm{~m})$. The test room floor slab is elevated to accommodate a lower plenum beneath the floor, and all other room surfaces are adjacent to temperature-controlled guard air spaces. Duplicate lighting and HVAC systems are installed in both the test room plenum and the lower plenum. Figure 4 shows a cut-away schematic view of the test facility. The majority of the ductwork is not shown in this figure.

The test room floor and ceiling slabs are $21 / 2$ inch $(63.5 \mathrm{~mm})$ thick concrete built on steel decks supported by a structural steel framework. The test room walls are constructed of gypsum drywall on steel studs. The initial test configuration is four interior walls.

The lighting system locations in the test room and lower plenum are identical. The edges of the floor slabs extend slightly beyond the walls, while the ceiling slab separates the side guard air spaces from the upper guard air space. Small access doors allow entry into the 
guard air spaces. A large double door opens from the north guard air space. Another door connects the test room/guard air portion of the test facility with steps leading to the floor of the control room.

The operation of the test facility, data collection and control of measurements is by a personal computer based data acquisition and control system. The system consists of an IBM AT and three Keithly Series 500 units. The computer collects data and controls the heaters and fan and other measurement parameters under the control of a specialized computer program written in SOFT500, an extended version of BASIC. A total of 398 parameters are sampled every 12 seconds and recorded at two minute intervals.

The primary measurement parameters are lighting power, cooling load, return airflow rate and room temperature. Additional measurements of air and surface temperatures, heat flows and light levels are intended to supply supporting information. Some of the measurements also serve as feedback signals for the control loops, such as the floor and wall thermopiles.

Lighting power is measured using a solid state transducer with a current output proportional to power consumption. This sensor was calibrated at NIST and found to have an uncertainty of less than 0.1 percent.

The cooling load for the test room is given by:

$$
\mathrm{Q}=\mathrm{mC}_{\mathrm{p}} \Delta \mathrm{T}=(\mathrm{AF})(\rho)\left(\mathrm{C}_{\mathrm{p}}\right)\left(\mathrm{T}_{\mathrm{RET}}-\mathrm{T}_{\mathrm{SUP}}\right)
$$

$$
\text { where: } \quad \begin{array}{ll}
\mathrm{m} & =\text { mass flow rate of air } \\
\rho & =\text { density of standard air } \\
\mathrm{C}_{\mathrm{p}} & =\text { specific heat of air } \\
\mathrm{T}_{\mathrm{RET}} & =\text { return air temperature } \\
\mathrm{T}_{\mathrm{SUP}} & =\text { supply air temperature }
\end{array}
$$

The test room air temperature is measured with an array of 64 thermocouples, in a four by four by four grid. The upper 16 thermocouples are in the plenum, while the average of the 48 thermocouples below the suspended ceiling is used as the room temperature control point. Temperature measurement uncertainty is $0.75^{\circ} \mathrm{F}\left(0.42^{\circ} \mathrm{C}\right)$.

Thermopiles are used extensively to control the test room boundary conditions. The most typical boundary condition is to simulate a test room surrounded by similar spaces. Thus, wall temperature conditions would be symmetric about the center plane of each test room wall, and the surface temperature would remain equal on both sides of the wall. The average temperature difference across each wall is measured using Type $T$ thermopiles with 30 pairs of junctions. The control system attempts to keep the thermopile readings equal to zero by varying the power supplied to each electric duct heater. When the lights are switched on, the interior of the test room walls begin to heat up, causing an imbalance in surface temperatures 
across the walls which is sensed by the thermopile which, in turn, causes the control signal to the appropriate heater to increase. In this manner, heat can be stored in the gypsum board walls, but no net heat flow through the wall will occur. The control of the lower plenum and upper guard air space is also accomplished using thermopiles.

Both steady state and transient tests can be run in the test facility. Steady state tests involve establishing a test configuration and allowing conditions to stabilize. The transient tests involve the response of cooling load due to sudden switching of the lighting system as shown in figure 5. Thus, the test facility goes from one steady state condition, either lights on or off, to the other steady state condition. Periodic operation of the lighting and/or internal electric equipment is also possible.

The test facility is essentially a guarded calorimeter being operated in a calibrated mode, with the test room functioning as a calorimeter. The design and method of operation is similar to the NRC test facility [5] which was used to determine the lighting cooling load factors which are contained in the ASHRAE Handbook of Fundamentals [6]. The procedures for operating a guarded calorimeter are detailed in ASTM Standard C-236. Guard air spaces are used to minimize unwanted heat transfer. However, some unwanted heat transfer will occur for large test facilities. Thus, the principles of operating a calibrated hot box, as described in ASTM Standard C-976 are utilized. This involves correcting the measured cooling loads by subtracting any excess heat gains or losses as determined through calibration. The steady-state lights-off and lights-on conditions provide the two calibrated points needed to calibrate the cooling load measurement. The metered lighting input power functions as the cooling load reference.

Examples of measurement data are shown in the following series of figures, for a transient lights-on test. Figure 6 shows cooling load, lighting power and supply-return airflow rate. The test room was initially at equilibrium with the lights off. At time zero the lighting system was energized and the room cooling load begun to increase. At the same time, the plenum air temperature increases, as shown in figure 7, while room air temperature is held constant. Heat storage in the floor and ceiling slabs causes their temperature to increase, as shown in figure 8. Similarly, the plenum wall temperature increases more than the room walls, as shown in figure 9. The fluorescent lamps heat up very quickly, as shown in figure 10.

The measured cooling load is fitted with a double exponential curve as shown in figure 11. The exponential curve is a compact way to represent the measured data, and leads to the weighting factors. The details of the double exponential curve fit and derivation of the extended weighting factors is given in [3]. 


\section{Results}

Table 1 summarizes the basic testing configurations. The primary test parameters are luminaire type, return air path and lamp type. The items listed under sensitivity tests were varied to determine their impact on lighting and HVAC system performance.

\section{Table 1 - Basic Test Configurations}

Lamps

$4 @ 40 \mathrm{~W}$

$4 @ 40 \mathrm{~W}$

$4 @ 40 \mathrm{~W}$

$4 @ 40 \mathrm{~W}$

$4 @ 40 \mathrm{~W}$

$2 @ 40 w$

$2 @ 40 w$

$2 @ 40 w$

2 @40w

$2 @ 40 w$

$2 @ 34 W$

$2 @ 34 W$
Luminaire

4 parabolic

4 parabolic

4 parabolic

4 acrylic lens

4 acrylic lens

4 acrylic lens

4 acrylic lens

4 acrylic lens

3 acrylic lens

3 acrylic lens

3 acrylic lens

3 acrylic lens
Return Air Path

ceiling grill

side slots

lamp compartment

ceiling grill

lamp compartment

ceiling grill lamp compartment static plenum

ceiling grill

lamp compartment

ceiling grill

lamp compartment

\section{Sensitivity Tests}

Carpet

Furnishings

Auxiliary Electric

Airflow Rate

Room Air Temperature

Controls 
The measurement results fall generally into two categories, those related to lighting system performance and efficiency, and those related to cooling load profiles. Of course, there is some interdependence between these two categories, since lighting efficiency influences cooling loads. However, even for a fixed lighting system efficiency, considerable variation in peak cooling load due to lighting can occur as a function of HVAC system design and operation, and room configuration.

Before the detailed measurement results are presented, a summary of the general results and trends will be discussed. The basis for these results and supporting information will follow. Some of the general results will likely seem to follow common sense, as they should. Their impact lies in the detailed quantitative information which verified their significance and the magnitude of their influence. A number of general trends and conclusions can be identified upon examining the measured results.

One major finding is that while lamp light output, power input and efficiency vary with temperature over a large range, the range of lamp temperatures which can be expected to occur under typical operating conditions is very limited for a particular luminaire type. The possible range of lamp temperatures varies with luminaire type, but for a particular luminaire minimum lamp temperatures elevation above room temperature will lie within a band of about 6 to $10^{\circ} \mathrm{F}\left(3.3\right.$ to $\left.5.5^{\circ} \mathrm{C}\right)$ for an extreme range of operating conditions. That is, as airflow rate, air temperature and airflow configuration are varied, minimum lamp temperature elevation over room air temperature varies only 6 to $10^{\circ} \mathrm{F}\left(3.3\right.$ to $\left.5.5^{\circ} \mathrm{C}\right)$. Thus, a 4-lamp fixture with an acrylic lens will always operate on the too warm side of the lamp efficiency curve. Energy efficiency and light output can be improved using lamp compartment return, but optimum lamp temperatures cannot be achieved under reasonable operating conditions. At the other extreme, a 2-lamp luminaire with $34 \mathrm{~W}$ lamps will always be too cool if lamp compartment return is used. The implication for design is that each luminaire type has a particular limited range of operating temperatures. The luminaire type and HVAC design must be selected so that the optimum lamp temperature can be achieved. Methods for doing this are described in the detailed results.

Another finding is that airflow rate has a limited impact on cooling load profiles, and thus, peak cooling loads due to lighting. Peak cooling loads are primarily dependent on the long term heat storage characteristics of the room, primarily the floor and ceiling slabs. Unless other methods of external heat storage are implemented, such as rock beds or ice storage, or control strategies are used, peak cooling loads cannot be altered to a great extent.

The use of carpet is beneficial because it allows the floor slab to heat up more, thereby storing more heat and slowing down the pickups of cooling load. The plenum area is the best place to store the heat from the lighting system for a number of reasons. First, both the lighting system (heat source) and floor slabs (heat storage) are in contact with the plenum. Second, there may be other structural elements in the plenum for additional heat storage. Third, the plenum can heat up without disturbing the building occupants in the conditioned space. 
The effect of furnishings, such as desks, chairs and file cabinets, is to slightly speed up the pickup of cooling load due to lights. The furnishings absorb radiant energy from the lighting system and transfer it to the room air by convection. Although the furnishings add more thermal mass to the room, it is in the conditioned space where it cannot heat up much, thereby allowing little additional heat storage.

The following sections describe more detailed measurement results.

\subsection{Lighting System Performance and Efficiency}

As was mentioned in the general results, each luminaire type responds to the thermal environment provided by the room and HVAC system in a characteristic manner.

Equilibrium minimum lamp temperatures vary with room air temperature, airflow rate and return air path. As lamp temperatures change, so do light output and power consumption. Measurements were made of average minimum lamp temperatures, light output and power consumption at equilibrium for a number of different room and HVAC conditions likely to be encountered in office buildings. Room air temperature was varied from 70 to $80^{\circ} \mathrm{F}(21.1$ to $26.6^{\circ} \mathrm{C}$ ), while airflow rate was varied from 120 to $200 \mathrm{cfm}\left(.0566\right.$ to $\left..0944 \mathrm{~m}^{3} / \mathrm{s}\right)$ or from 0.7 to $1.2 \mathrm{cfm} / \mathrm{ft}^{2}$ of floor area $\left(.00356\right.$ to $.0061 \mathrm{~m}^{3} / \mathrm{s} / \mathrm{m}^{2}$; i.e., .00356 to $.0061 \mathrm{~m}^{3} / \mathrm{s}$ air flow per $\mathrm{m}^{2}$ of floor area). Return air was extracted from the room either through a ceiling grill or through the luminaires (lamp compartment return). In one case, return air was extracted through slots around the perimeter of the luminaires (side slot return), but this was found to be very similar to ceiling grill return.

Table 2 presents a summary of lamp temperature results for the various lighting systems. The elevation of minimum lamp temperature is reported for each configuration. These include various airflow rates and room air temperatures. Three values are tabulated, including the minimum, mean and maximum elevation of minimum lamp wall temperature above room air temperature.

The results in Table 2 indicate that the acrylic lens luminaire operated about 10 to $15^{\circ} \mathrm{F}(5.7$ to $8.3^{\circ} \mathrm{C}$ ) warmer than the open cell parabolic luminaire, due to the entrapment of heat by the acrylic lens. Lamp compartment return reduce minimum lamp temperatures by $10^{\circ} \mathrm{F}$ $\left(5.67^{\circ} \mathrm{C}\right)$ for the parabolic luminaire, and 8 to $10^{\circ} \mathrm{F}\left(4.4\right.$ to $\left.5.7^{\circ} \mathrm{C}\right)$ for the acrylic lens luminaire. The relatively open design of the parabolic diffuser took better advantage of the cooling effect of the lamp compartment venting.

The two lamp acrylic luminaires operated about 7 to $12^{\circ} \mathrm{F}\left(3.9\right.$ to $\left.6.7^{\circ} \mathrm{C}\right)$ cooler than a similar four lamp luminaire, with $40 \mathrm{~W}$ lamps. The $34 \mathrm{~W}$ lamps were substantially cooler than the $40 \mathrm{~W}$ lamps, being lower by 8 to $10^{\circ} \mathrm{F}\left(4.4\right.$ to $\left.5.7^{\circ} \mathrm{C}\right)$. This reduction in temperature was significantly greater than the ratio of power input, which was about 85 percent, and merits further study.

Lamp temperatures were very similar for the ceiling grill and side slot air returns. Evidently, the cooling effect of the return air requires lamp compartment extract. 
Table 2 - Lamp Temperature Elevations

\begin{tabular}{|c|c|c|c|c|c|}
\hline \multirow{3}{*}{$\begin{array}{l}\text { Number } \\
\text { of Lamps }\end{array}$} & \multirow{3}{*}{$\begin{array}{l}\text { Luminaire } \\
\text { Type }\end{array}$} & \multirow{3}{*}{$\begin{array}{c}\text { Return } \\
\text { Air Path }\end{array}$} & \multicolumn{3}{|c|}{ Minimum Lamp ${ }^{\circ} \mathrm{F}\left({ }^{\circ} \mathrm{C}\right)$} \\
\hline & & & \multicolumn{3}{|c|}{ Temperature Elevation } \\
\hline & & & $\underline{\text { Minimum }}$ & Mean & $\underline{\text { Maximum }}$ \\
\hline $4 @ 40 w$ & Parabolic & ceil.grill & $37.1(2.81)$ & $38.5(3.61)$ & $39.3(4.06)$ \\
\hline $4 @ 40 w$ & Parabolic & side slots & $36.0(2.22)$ & $37.6(3.11)$ & $39.4(4.11)$ \\
\hline $4 @ 40 w$ & Parabolic & lamp comp. & $24.9(-3.94)$ & $27.9(-2.28)$ & $30.9(-.61)$ \\
\hline $4 @ 40 w$ & Acry. lens & ceil.grill & $46.6(8.11)$ & $48.4(9.11)$ & $50.5(10.28)$ \\
\hline $4 @ 40 w$ & Acry. lens & lamp comp. & $40.3(4.61)$ & $42.8(6.0)$ & $44.8(7.11)$ \\
\hline $2 @ 40 w$ & Acry. lens & ceil. grill & $38.5(3.61)$ & $40.4(4.67)$ & $43.2(6.22)$ \\
\hline $2 @ 40 W$ & Acry. lens & lamp comp. & $27.5(-2.5)$ & $30.9(-.61)$ & $34.5(1.39)$ \\
\hline $2 @ 34 W$ & Acry. lens & ceil.grill & $28.2(-2.11)$ & $30.8(-.67)$ & $33.0(.56)$ \\
\hline $2 @ 34 W$ & Acry. lens & lamp comp. & $18.8(-7.33)$ & $22.5(-5.28)$ & $25.3(-3.72)$ \\
\hline
\end{tabular}

\subsubsection{Four Lamp Acrylic Lens Luminaire}

As would be expected, the 4 lamp fixtures operate at the warmest temperatures. This is especially true for the acrylic lens fixture, which encloses the lamps between the housing and the lens. Measurements of lighting power input, light output and lamp temperature were made for a number of airflow rates and room air temperatures. Airflow rates ranged from approximately 120 to $200 \mathrm{cfm}\left(.0566\right.$ to $\left..0944 \mathrm{~m}^{3} / \mathrm{s}\right)$, and room air temperature was controlled to 70,75 and $80^{\circ} \mathrm{F}\left(21.1,23.9\right.$ and $\left.26.7^{\circ} \mathrm{C}\right)$. The minimum lamp temperature was defined as the average of the individual luminaire lamp temperature minima. There were four luminaires, each with four $40 \mathrm{~W}$ lamps, for a total of 16 lamps.

Figure 12 shows the measured average minimum lamp wall temperatures (AMLWT) for the 4 lamp acrylic lens luminaire. Two air return paths are included; ceiling grill return (CG) and lamp compartment extract (LC). In both cases, the luminaires were mounted in a vented 
ceiling plenum, flush with the suspended ceiling. AMLWT varied from $109^{\circ} \mathrm{F}$ to $129^{\circ} \mathrm{F}$ $\left(42.8\right.$ to $\left.53.9^{\circ} \mathrm{C}\right)$ over all of the tests. With the ceiling grill return, AMLWT is always above $118^{\circ} \mathrm{F}\left(47.8^{\circ} \mathrm{C}\right)$, usually about 5 to $8^{\circ} \mathrm{F}\left(2.8\right.$ to $\left.4.4^{\circ} \mathrm{C}\right)$ warmer than with lamp compartment return.

In all cases, the lamps are warmer than the optimum temperature of about $104^{\circ} \mathrm{F}\left(40^{\circ} \mathrm{C}\right)$. Only the lamp compartment return with a room air temperature of $70^{\circ} \mathrm{F}\left(21.1^{\circ} \mathrm{C}\right)$ approaches energy efficient lamp temperature conditions.

Lamp temperatures are more sensitive to airflow rate with lamp compartment return than with ceiling grill return, since the return air is more strongly linked convectively to the lamps when it passes through the lamp compartment. This effect can be seen more readily in figure 13, which shows the elevation of AMLWT above room air temperature for the various test conditions. With ceiling grill return, AMLWT elevation is about 47 to $51^{\circ} \mathrm{F}$ ( 8.33 to $10.6^{\circ} \mathrm{C}$ ), with one exception. There is only a slight cooling effect associated with increased airflow. With lamp compartment return, on the other hand, AMLWT is about $5^{\circ} \mathrm{F}\left(2.78^{\circ} \mathrm{C}\right)$ cooler at the highest airflow rate than at the lowest, ranging from about 40 to $45^{\circ} \mathrm{F}$ ( 4.4 to $7.2^{\circ} \mathrm{C}$ ). The effect of room air temperature is normalized by plotting AMLWT elevation. The AMLWT elevation can be used to estimate lamp temperatures for various room air temperatures and air return configurations, by adding the AMLWT elevation to the desired room air temperature.

Figure 14 shows the variations in lighting power, light output and luminous efficacy which occurred at the various test conditions, plotted versus AMLWT for the ceiling grill return. Figure 15 shows similar data for the lamp compartment return. In each plot, values are normalized to the maximum values for each condition (i.e. relative lighting power-LP, relative light output-LD, relative luminous efficacy-LE). It is clear that for the ceiling grill return the lamps are operating in the overheated range, since lower AMLWT's provided higher lighting power and light output. Efficiency varies by 10 percent, and light output by nearly 12 percent for the various test conditions.

For the lamp compartment return, performance is better, with only 5 percent variations apparent in luminous efficacy. This indicates that though the average of the minimum lamp wall temperatures may be above the $104^{\circ} \mathrm{F}\left(40^{\circ} \mathrm{C}\right)$ optimum, some of the individual lamp minimum lamp wall temperatures may be near the optimum. Lighting power and light output are monitored for the ensemble of 16 lamps, each of which can have a unique local thermal environment, and consequently, temperature distribution.

Figure 16 compares relative luminous efficacy for the lamp compartment and ceiling grill returns as functions of AMLWT. This shows that although AMLWT's are similar for some of the test conditions, efficiencies are higher for the lamp compartment return, and not as variable. 
These results indicate that lamp compartment return is preferable for 4 lamp, acrylic lens luminaires, which would otherwise operate at lamp temperatures that are too high.

\subsubsection{Four Lamp Parabolic Diffuser Luminaire}

The measured AMLWT and temperature elevations for the 4 lamp parabolic luminaires are shown in figures 17 and 18, respectively. AMLWT's range from 97 to $116^{\circ} \mathrm{F}(36.1$ to 46.7 ${ }^{\circ} \mathrm{C}$ ) for all of the conditions. In addition to the lamp compartment and ceiling grill air returns, a side slot air return was tested. In the side slot configuration, air is drawn from the room into the plenum return through slots around the perimeter of each luminaire. Lamp temperatures were found to be very similar for both ceiling grill and side slot air returns, ranging from 112 to $116^{\circ} \mathrm{F}\left(44.4\right.$ to $\left.46.7^{\circ} \mathrm{C}\right)$ for a room air temperature of $75^{\circ} \mathrm{F}\left(23.9^{\circ} \mathrm{C}\right)$ (the only room air temperature tested). Lamp temperatures varied more widely for lamp compartment return, primarily due to the wide range of room air temperature conditions, but also due to the influence of airflow rate. AMLWT elevations above room air temperature were about 25 to $31^{\circ} \mathrm{F}\left(-3.9\right.$ to $\left.-.6^{\circ} \mathrm{C}\right)$ for lamp compartment return, and 36 to $40^{\circ} \mathrm{F}$ ( 2.2 to $4.4^{\circ} \mathrm{C}$ ) for ceiling grill and side slot returns.

The relative performance characteristics of the 4 lamp parabolic luminaire are shown in figures 19,20 and 21 for ceiling grill, side slots and lamp compartment returns, respectively. These figures show that with ceiling grill and side slot air returns, lamp temperatures are warmer than optimum, although only a slight effect ( $\sim 2$ percent) on efficiency and light output was observed. This effect was small due to the narrow range of lamp temperatures induced by the single room air temperature of $75^{\circ} \mathrm{F}\left(23.9^{\circ} \mathrm{C}\right)$. A wider range of room temperatures, such as was used for the lamp compartment return, would have produced greater variations. Efficiency varies by almost 5 percent for the lamp compartment return. Lamp temperatures are clustered around the optimum of $104^{\circ} \mathrm{F}\left(40^{\circ} \mathrm{C}\right)$.

Figure 22 compares the relative luminous efficacies for the three return air paths.

Considerable scatter is apparent for the lamp compartment return results, due to measurement noise. This problem was corrected following the lamp compartment return tests. However, based on the measured lamp temperature ranges, the lamp compartment return appears to be near the optimum lamp temperature, while the ceiling grill return is slightly warmer.

These results indicate that the 4-lamp parabolic luminaire is more efficient than a similar luminaire with an acrylic lens, due to cooler lamp temperatures. Lamp compartment air return is slightly better than ceiling grill or side slot returns, which are very similar in performance. 


\subsubsection{Two Lamp Acrylic Lens Luminaire}

Three different configurations were tested for the 2 lamp acrylic lens luminaire. Two of the configurations consisted of three fixtures with either 34 or $40 \mathrm{~W}$ lamps. The third configuration consisted of four fixtures with $40 \mathrm{~W}$ lamps. These combinations were selected to enable comparison of identical luminaire configurations with different wattage lamps, and identical luminaire types with different numbers of luminaires.

It might be expected that a luminaire with higher wattage lamps would operate with warmer lamp temperatures, due to the higher power input. It is also possible that four luminaires will perform differently than three due to differences in supply air temperature and airflow rate per luminaire. This is particularly true for the lamp compartment air return where for the three luminaire configurations roughly one third of the return air is extracted through each luminaire, compared to one fourth for the four luminaire configurations.

Figure 23 shows AMLWT for the four-luminaire configuration, while figure 24 shows the temperature elevations. AMLWT ranged from 96 to $119^{\circ} \mathrm{F}\left(35.6\right.$ to $\left.48.3^{\circ} \mathrm{C}\right)$, translating into temperature elevations of about $30^{\circ} \mathrm{F}\left(16.7^{\circ} \mathrm{C}\right)$ for lamp compartment return, and $40^{\circ} \mathrm{F}$ $\left(22.2^{\circ} \mathrm{C}\right)$ for ceiling grill return. The relative performance characteristics for the ceiling grill return are shown in figure 25 , and lamp compartment return in figure 26. Lamp temperatures are warmer than optimum for the ceiling grill return, but centered about the optimum for lamp compartment return. The relative luminous efficacies of the two return configuration are compared in figure 27.

Lamp temperatures were slightly cooler for the three luminaire configuration than the fourluminaire configuration with $40 \mathrm{~W}$ lamps, as shown in figures 28 and 29. This is probably due to the lower lighting power per supply airflow rate. The lamp compartment return cools the lamps about $6^{\circ} \mathrm{F}\left(3.3^{\circ} \mathrm{C}\right)$ below ceiling grill return. The relative performance characteristics are shown in figure 30 for the ceiling grill return and figure 31 for the lamp compartment return. Both conditions are near the optimum temperature range, although the ceiling grill appears to be on the too warm side of the efficacy peak. In both cases, efficiency varies by less than $3 \%$ over the range of conditions tested. Figure 32 compares the luminous efficacies for the two return air configurations.

The final configuration consists of three luminaires with $34 \mathrm{~W}$ lamps. Figures 33 and 34 show lamp temperatures and temperature elevations. Lamp temperatures are too cool for the lamp compartment return, rising above room air temperature by only 20 to $25^{\circ} \mathrm{F}$ (11.1 to $13.9^{\circ} \mathrm{C}$ ). Lamp temperatures with the ceiling grill return, however, are nearly optimum, about 29 to $33^{\circ} \mathrm{F}\left(16.1\right.$ to $\left.18.3^{\circ} \mathrm{C}\right)$ warmer than room air. This effect is clear in the relative performance plots in figures 35 and 36 . Luminous efficacy peaks in the middle of the ceiling grill return temperature range, while efficiency for the lamp compartment return is reduced by nearly $20 \%$ in the worst case. Light output is also down $23 \%$. The lamp compartment return is clearly over-cooling the lamps. Figure 37 compares the luminous efficacies of the two return configurations. 


\subsection{Lighting Cooling Load Regression Analysis}

The response of cooling load to a step change in lighting forms the basis of the evaluation of cooling load due to dynamic lighting system operation. The measured cooling loads due to lighting were fit with double exponential relations, which represent a compact form of describing the step response of cooling load due to lighting. Weighting factors were computed from the regression coefficients, and subsequently, cooling loads due to cyclic (i.e. daily) operation of the lighting system were determined. The peak cooling loads during cyclic operation of light were extracted from the periodic cooling load profiles.

The evaluation included the effect on cooling load of luminaire type, airflow rate, return air path and other parameters such as carpeting and furnishings.

Airflow rate was varied through five ranges, though not for every test. Most configurations were measured at the middle three airflow rates, with additional tests at very low or very high airflows. Table 3 summarizes the airflow ranges used in the testing, expressed as total airflow, airflow per floor area and airflow per luminaire.

Table 3 - Airflow Rates for Testing

\begin{tabular}{|c|c|c|c|c|}
\hline \multirow[t]{2}{*}{$\begin{array}{l}\text { Airflow } \\
\text { Range }\end{array}$} & \multirow[t]{2}{*}{$\begin{array}{l}\text { Total Airflow, } \\
\mathrm{cfm}\left(\mathrm{m}^{3} / \mathrm{s} \times 10^{-3}\right)\end{array}$} & \multirow[t]{2}{*}{$\begin{array}{l}\text { Airflow per floor area, } \\
\mathrm{cfm} / \mathrm{ft}^{2}\left(\mathrm{~m}^{3} / \mathrm{s}^{2} \mathrm{~m}^{2} \times 10^{-3}\right)\end{array}$} & \multicolumn{2}{|c|}{$\begin{array}{l}\text { Airflow per Luminaire, } \\
\mathrm{cfm}\left(\mathrm{m}^{3} / \mathrm{s} \times 10^{-3}\right)\end{array}$} \\
\hline & & & Four Luminaires & Three Luminaires \\
\hline Very Low & $85-96(40.1-45.3)$ & $0.51-0.57(2.59$ & $21-24(9.9-11.3)$ & $28-32(13.2-15.1)$ \\
\hline Low & $127-144(59.9$ & 0.76 & $32-36(15$. & $42-48(19.8-22.7)$ \\
\hline Medium & $169-192(79.8-90.6)$ & $1.01-1.14(5.13-5.79)$ & $42-48(19.8-22.7)$ & $56-64(26.4-30.2)$ \\
\hline High & $214-240(101-113)$ & $1.27-1.43(6.45-7.26)$ & $54-60(25.5-28.3)$ & $71-80(33.5-37.8)$ \\
\hline Very High & $(151)$ & $(9.70)$ & $(37.8)$ & $(50.5)$ \\
\hline
\end{tabular}

The testing airflows are given in ranges due to variations in airflow settings and control system operation over the course of the testing. Individual tests were conducted at constant airflow rates, within the ranges listed.

Table 4 summarizes the measured cooling load results for all of the configurations. It includes both the exponential regression coefficients $(A, B, C$ and $D)$ and the weighting factors $\left(a_{1}, a_{2}, b_{1}, b_{2}\right)$. Cooling load due to a positive step change (turn lights on) in lighting is given by:

$$
\frac{Q}{W}=1-A e^{-B t}-C e^{-D t}
$$

and for a negative step change (turn lights off) by: 


$$
\frac{Q}{W}=A e^{-B t}+C e^{-D t}
$$

where $Q \quad=$ cooling load due to lighting

$$
\begin{array}{ll}
W & =\text { lighting power } \\
t & =\text { time } \\
A, B, C, D & =\text { regression coefficients }
\end{array}
$$

Cooling load due to cyclic operation of the lighting system is given by:

$$
\frac{Q}{W}(T)=a_{1} W(T-1)+a_{2} W(T-2)+b_{1} Q(T-1)-b_{2} Q(T-2)
$$

where

$$
T=\text { discrete time step }
$$

$$
\begin{aligned}
& a_{1}, a_{2}, b_{1}, b_{2}=\text { weighting factors } \\
& \qquad \begin{aligned}
a_{1} & =A e^{-D}+A+C e^{-B}+C-e^{-D}-e^{-B} \\
a_{2} & =e^{-B-D}-A e^{-D}-C e^{-B} \\
b_{1} & =e^{-D}+e^{-B} \\
b_{2} & =e^{-B-D}
\end{aligned}
\end{aligned}
$$

\subsubsection{Four Lamp Parabolic Luminaire}

The four lamp parabolic luminaires were the first fixtures tested. The initial room configuration was uncarpeted and unfurnished. Air was supplied to the room at ceiling height through two perforated supply air diffusers. Return air left the conditioned space via the plenum through either a ceiling grill, luminaire side slots or lamp compartment.

Figure 38 presents the cooling load regression curves for lamp compartment return. Similar results are shown in figure 39 for ceiling grill return and figure 40 for side slot return. Each plot includes three airflow rates, low (nominally $120 \mathrm{cfm}$ or $.0566 \mathrm{~m}^{3} / \mathrm{s}$ ), medium (nominally $160 \mathrm{cfm}$ or $.0755 \mathrm{~m}^{3} / \mathrm{s}$ ), and high (nominally $200 \mathrm{cfm}$ or $.0944 \mathrm{~m}^{3} / \mathrm{s}$ ). Despite the wide 
Table 4 - Measured Cooling Load Results

4 × 4 Parabolic 40W Side Slot Return/Unfurnished/No Carpet

$\begin{array}{lcccccccc}\text { Airflow } & \text { A } & \text { B } & \text { C } & \text { D } & \text { a1 } & \text { a2 } & \text { b1 } & \text { b2 } \\ \text { Very Low } & 0.75383 & .24684 & .24617 & 4.28598 & .407671 & -.191947 & .795026 & .010750 \\ \text { Low } & 0.72832 & .24733 & .27168 & 5.54895 & .43021 & -.211946 & .784775 & .003039 \\ \text { Medium } & 0.62282 & .22851 & .37718 & 2.94023 & .484476 & -.290991 & .848572 & .042057\end{array}$

$4 \times 4$ Parabolic $40 \mathrm{~W}$ Ceiling Grill Return/Unfurnished/No Carpet

$\begin{array}{lcccccccc}\text { Airflow } & \text { A } & \text { B } & \text { C } & \text { D } & \text { a1 } & \text { a2 } & \text { b1 } & \text { b2 } \\ \text { Low } & 0.6633 & .2245 & .3367 & 2.88835 & .451336 & -.261445 & .854584 & .044474 \\ \text { Medium } & 0.5409 & .1833 & .4591 & 1.8362 & .476500 & -.335719 & .991940 & .132722 \\ \text { High } & 0.5708 & .2048 & .4292 & 2.4340 & .497272 & -.328320 & .902496 & .071447\end{array}$

$4 \times 4$ Parabolic 40W Lamp Compartment Return/Unfurnished/No Carpet

$\begin{array}{llllcrrrr}\text { Airflow } & \text { A } & \text { B } & \text { C } & \text { D } & \text { al } & \text { a2 } & \text { b1 } & \text { b2 } \\ \text { Low } & 0.6286 & .1856 & .3714 & 1.6686 & .407868 & -.270407 & 1.019117 & .156578 \\ \text { Medium } & 0.5853 & .1838 & .4147 & 3.0075 & .492478 & -.332877 & .881517 & .041118 \\ \text { High } & 0.63385 & .2237 & .36615 & 4.243 & .487943 & -.290377 & .813919 & .011485\end{array}$

4 x 4 Parabolic 40W Lamp Compartment Return/Unfurnished/With Carpet

\begin{tabular}{llllccccc} 
Airflow & \multicolumn{1}{c}{ A } & B & C & D & al & a2 & b1 & b2 \\
Low & 0.4812 & .1414 & .5188 & 2.420 & 0.536118 & -.415985 & .957064 & .077197 \\
Medium & 0.48355 & .16515 & .50645 & 3.333 & .563512 & -.416711 & .883452 & .030253 \\
High & 0.5308 & .2017 & .4692 & 4.282 & .559674 & -.379537 & .831155 & .011292
\end{tabular}

$4 \times 4$ Parabolic 40W Side Slot Return/Unfurnished/With Carpet

\begin{tabular}{lllcccccc} 
Airflow & \multicolumn{1}{c}{ A } & B & C & D & al & a2 & b1 & b2 \\
Very low & 0.4832 & .1126 & .5163 & 2.136 & .506262 & -.412850 & 1.011634 & .105547 \\
Low & 0.497 & .1669 & .503 & 2.982 & .553899 & -.407975 & .896976 & .042899 \\
High & 0.4836 & .1818 & .5164 & 5.116 & .593691 & -.438457 & .839768 & .005003
\end{tabular}

$4 \times 2$ Acrylic 40W Ceiling Grill Return, Sealed Luminaires/Unfurnished w/Carpet

$\begin{array}{lcccccccc}\text { Airflow } & \text { A } & \text { B } & \text { C } & \text { D } & \text { a1 } & \text { a2 } & \text { b1 } & \text { b2 } \\ \text { Low } & 0.4218 & .1157 & .5783 & 1.6449 & .512863 & -.4246 & 1.08377 & .171942 \\ \text { Medium } & 0.3775 & .1242 & .6225 & 1.7945 & .563125 & -.46574 & 1.04941 & .146798 \\ \text { High } & 0.4114 & .1524 & .5886 & 2.1926 & .581050 & -.455474 & .970271 & .095847\end{array}$


$4 \times 2$ Acrylic 40W Lamp Compartment Return/Unfurnished w/Carpet

$\begin{array}{lcccccccc}\text { Airflow } & \text { A } & \text { B } & \text { C } & \text { D } & \text { al } & \text { a2 } & \text { b1 } & \text { b2 } \\ \text { Low } & 0.4732 & .1319 & .5268 & 1.9111 & .50735 & -.402058 & 1.02435 & .129639 \\ \text { Medium } & 0.4472 & .1533 & .5528 & 2.0510 & .545266 & -.421417 & .986479 & .110328 \\ \text { High } & 0.4449 & .1931 & .5552 & 2.6910 & .595764 & -.431972 & .89243 & .055905\end{array}$

$4 \times 2$ Acrylic 40W Ceiling Grill Return/Unfurnished w/Carpet

$\begin{array}{lcccccccc}\text { Airflow } & \text { A } & \text { B } & \text { C } & \text { D } & \text { a1 } & \text { a2 } & \text { B1 } & \text { B2 } \\ \text { Low } & 0.4683 & .1388 & .5317 & 2.0243 & .522160 & -.409681 & 1.002488 & .114968 \\ \text { Medium } & 0.4106 & .1350 & .5894 & 1.9415 & .556680 & -.448516 & 1.017204 & .125368 \\ \text { High } & 0.4366 & .1671 & .5634 & 2.4887 & .583814 & -.442704 & .92913 & .070243 \\ \text { Very High } & 0.4494 & .2945 & .5506 & 3.7720 & .652573 & -.403346 & .76791 & .017137\end{array}$

$4 \times 4$ Acrylic 40W Ceiling Grill Return/Unfurnished/With Carpet

$\begin{array}{lcccccccc}\text { Airflow } & \text { A } & \text { B } & \text { C } & \text { D } & \text { a1 } & \text { a2 } & \text { b1 } & \text { b2 } \\ \text { Very low } & 0.5516 & .1577 & .4484 & 2.109 & .474458 & -.346269 & .975465 & .103654 \\ \text { Medium } & 0.4617 & .18524 & .5383 & 2.15335 & .553881 & -.404410 & .946991 & .096463\end{array}$

4 x 4 Acrylic 40W Lamp Compartment Return/Unfurnished/With Carpet

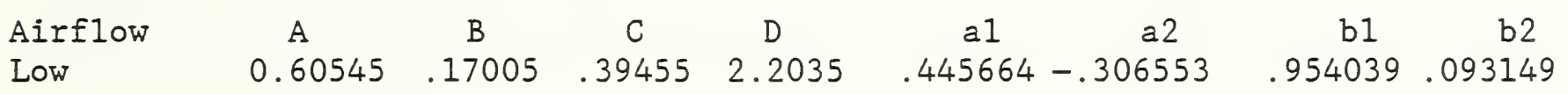

$4 \times 4$ Acrylic 40W Ceiling Grill Return, Perforated Supply/Unfurnished/With Carpet

$\begin{array}{llllccrrr}\text { Airflow } & \text { A } & \text { B } & \text { C } & D & \text { a1 } & \text { a2 } & \text { b1 } & \text { b2 } \\ \text { Very low } & 0.59975 & .16155 & .40025 & 1.9947 & .435263 & -.306383 & .986878 & .115758 \\ \text { Low } & 0.5486 & .1841 & .4514 & 2.035 & .484656 & -.338483 & .962533 & .108707 \\ \text { Medium } & 0.4895 & .2025 & .5105 & 1.9478 & .527441 & -.370266 & .959274 & .116449\end{array}$


range of airflows, the cooling load regressions vary only a small amount, except for the lamp compartment return. As would be expected, the higher airflow rates cause a faster initial rise in cooling load, due to convective heat transfer to the return air. About 24 hours were required to reach a new equilibrium condition.

The lack of sensitivity of cooling load to airflow rate is due to the type of supply diffuser, and the heat transfer characteristics of the room. With the two perforated supply diffusers, supply air enters the conditioned space at low velocity. Changing airflow rate has only a small effect on room convective heat transfer. Since heat storage in the room components occurs until convective heat loss equals radiant heat gain and the rate of heat storage determines the cooling load pickup, airflow rate has a small effect on cooling load.

The effect of airflow rate on cooling load is greatest for lamp compartment return because the return air passes near the lamps, influencing convective heat transfer. Figures 41 and 42 show the cooling load regressions for the four lamp parabolic lens luminaires with carpet, for lamp compartment return and side slot returns, respectively. The effect of airflow rate is more noticeable than for the no carpet configuration. This is primarily due to the reduction in the rate of convective heat transfer to the floor slab, and the increase in total heat storage in the slabs.

Ceiling grill return and lamp compartment return are compared in figures 43,44 and 45 for the three airflow rates, without carpet. The cooling load pickup for the lamp compartment return are slightly slower than for the ceiling grill return. This is due to greater heat storage in the plenum and ceiling slab with lamp compartment return. When the return air passes through the lamp compartment, heat is extracted from the luminaires and dumped into the plenum.

The effect of carpet is demonstrated in figures 46,47 and 48 , for lamp compartment return and three airflow rates. The initial pickup of cooling load is faster with carpet, as the carpet absorbs radiant energy from the lights and transfers heat to the room air by convection. The long term cooling load is slower with carpet, especially at the lowest airflow, due to the insulating effect of the carpet, which allows more heat storage in the slabs.

\subsubsection{Four Lamp Acrylic Lens Luminaires}

The four lamp acrylic lens luminaire was tested with carpet. Figure 49 shows the cooling load pickup for lamp compartment return at very low airflow. Figure 50 presents the results for ceiling grill return at low and high airflow rates. Figure 51 compares lamp compartment and ceiling grill returns at the lowest airflow rates. As was the case with the parabolic luminaires, the pickup of cooling load with lamp compartment return is slower than ceiling grill return. Figures 52 and 53 compare the acrylic and parabolic luminaires at low and high airflow rates. The pickup of cooling load is faster initially for the parabolic luminaire, but slower at later times. Evidently, the open cell diffuser design allows greater convective heat transfer initially, and more heat storage in the long term. 


\subsubsection{Two Lamp Acrylic Lens Luminaires}

The two lamp acrylic lens luminaire was tested under a variety of configurations. Both the perforated and louvered supply air diffusers were used, as well as with and without furnishings.

Figure 54 presents the cooling load regressions for the ceiling grill return with perforated supply diffuser, for three airflow rates. The pickup of cooling load is noticeably faster at the high airflow rate, $200 \mathrm{cfm}\left(.0944 \mathrm{~m}^{3} / \mathrm{s}\right)$. A similar airflow rate effect is shown in figure 55 for the same configuration except louvered supply air diffuser. The effect of airflow rate is stronger still for lamp compartment return.

Figure 56 shows the cooling load regressions for the lamp compartment return at three airflow rates, for the acrylic lens luminaire, with carpet and without furnishings, but with louvered supply diffuser. The effect of airflow rate is noticeably larger than the previous results for the perforated diffuser. Flow visualizations using smoke generators indicated that air velocity leaving the single louvered supply was much greater than for the two perforated air diffusers. The supply air was also directed along the ceiling by the louvered supply, rather than being dumped at low velocity into the center of the room.

Figures 57 and 58 show the effect of airflow rate on cooling loads with and without furnishings for the ceiling grill return, with the louvered supply air diffuser. The effect of airflow rate is similar for the two conditions. The influence of the furnishings is described below.

One additional configuration was tested, that of ceiling grill return with sealed luminaires. The effect of airflow rate was similar for this configuration, as is shown in figure 59.

Returning to the effect of furnishings, figures 60 and 61 compare the cooling load regressions for identical configurations except for the presence of furnishings. Figure 60 compares the results for airflow rates of low and very high, while figure 61 compares the medium and high airflow rates. In all cases, the pickup of cooling load is faster with the furnishings than without. This is due to the absorption of radiation by the furnishings and the subsequent convective heat transfer to the room air. While the furnishings add additional thermal mass to the room, this mass does not heat up much since it is in contact with the room air. Thus, the shading effect of the furnishings more than compensate for the additional heat storage capacity.

\subsection{Cooling Load Profiles}

While the previous section presented the cooling load regression results, which are useful for understanding the physical processes occurring during transient testing, the cooling load profile with cyclic operation of the lighting system is of greater interest to the building designer. Lighting systems are rarely left on until equilibrium conditions occur. More 
typically, the lighting system will be switched on in the morning and off in the evening. Under these conditions, both the shape of the cooling load profile, which determines energy for cooling, and the peak cooling load, which is related to equipment sizing, are of interest.

The results of the regression analysis were used to generate cooling load profiles for a twelve hour on -- twelve hour off lighting schedule. The cooling load profiles were plotted for each configuration. In addition, peak cooling loads due to lighting were determined for lights-on times of four, six, eight, ten and twelve hours per 24 hour period.

This section presents the cooling load profile results, while the following section shows the peak cooling load results.

\subsubsection{Four Lamp Parabolic Luminaire}

Figures 62, 63 and 64 present the cooling load profiles as functions of airflow rate for each of the return air configurations, without carpet, but with the perforated supply air diffuser. Airflow rate has the greatest effect for lamp compartment return (figure 62), while ceiling grill (figure 63) and side slots (figure 64) do not show much sensitivity to airflow rate. Similar results with carpet, figures 65 and 66 , show a greater effect of airflow rate.

\subsubsection{Four Lamp Acrylic Luminaire}

Figures 67 and 68 present the cooling load profiles for lamp compartment return and ceiling grill return, respectively. Both configurations are carpeted. Cooling loads are about 5 percent higher at the greater airflow rate with ceiling grill return.

\subsubsection{Two Lamp Acrylic Luminaire}

Figure 69 presents the cooling load profiles for ceiling grill return with the perforated supply diffuser. Airflow rate exerts about a five percent effect. Similar results are seen for lamp compartment return in figure 70, although for the louvered supply diffuser. The ceiling grill results with the louvered supply diffuser, without and with furnishings are shown in figures 71 and 72 , respectively. The wider range of airflows produces a ten percent effect. In contrast, the ceiling grill sealed luminaire results (figure 73 ) show only about a three percent variation in cooling load profile over the range of airflows.

This lack of sensitivity to airflow rate is due to the fact that the luminaires are sealed and thus are not strongly influenced by changes in airflow rate.

\subsection{Peak Cooling Loads due to Lighting}

Figure 74 summarizes the peak cooling loads due to lighting for the acrylic lens luminaire with lamp compartment return. It includes all of the airflow rates and both the two lamp and four lamp luminaires. Peak cooling loads vary from 60 to 95 percent of lighting power as 
lighting system on time varies from two to 12 hours. For any fixed lights-on period, peak cooling loads vary from 5 to 10 percent. The two and four lamp luminaires perform similarly, except for the short on-times where the four lamp fixtures are as much as five percent below the two lamp fixtures. That is, the ratio of cooling load to lighting load is five percent less, while the absolute cooling load is greater due to the greater lighting power.

Similar results are seen for the ceiling grill return in figure 75. At the very high airflow, only a two percent peak cooling load reduction occurs for the twelve hour lights on period. The other airflow rates have peak load reductions of five to ten percent.

The four lamp parabolic luminaire results are shown in figures 76,77 and 78 without carpet, and figures 79 and 80 with carpet. For the uncarpeted configurations, little sensitivity of peak cooling load to airflow rate is seen, except for lamp compartment return and very short lights-on periods. However, with carpeting, peak cooling loads varied by about five percent for the lamp compartment return, and over five percent for the side slot return.

Figures 81 and 82 show peak cooling loads for unfurnished and furnished configurations, for otherwise identical conditions. The effect of airflow is similar for the two conditions, but the peak cooling loads are greater for the furnished room by about two to four percent.

\section{Conclusions}

An extensive series of measurements of the interaction of lighting and HVAC systems under typical operating conditions have highlighted and quantified a number of effects. These effects influence both lighting system efficiency and cooling loads due to lighting.

Understanding the interactions between the lighting and HVAC systems is essential for designing efficient lighting systems and controlling peak cooling loads due to lighting. By taking advantage of these interactions, energy and cost savings can be achieved while maintaining adequate lighting conditions.

One major finding is that while lamp light output, power input and efficiency vary with temperature over a large range, the range of lamp temperatures which can be expected to occur under typical operating conditions is very limited for a particular luminaire type. The possible range of lamp temperatures varies with luminaire type, but for a particular luminaire minimum lamp temperatures elevation above room temperature will lie within a band of about 6 to $10^{\circ} \mathrm{F}\left(3.3\right.$ to $\left.5.5^{\circ} \mathrm{C}\right)$ for an extreme range of operating conditions. That is, as airflow rate, air temperature and airflow configuration are varied, minimum lamp temperature elevation over room air temperature varies only 6 to $10^{\circ} \mathrm{F}\left(3.3\right.$ to $\left.5.5^{\circ} \mathrm{C}\right)$. Thus, a 4-lamp fixture with an acrylic lens will always operate on the too warm side of the lamp efficiency curve. Energy efficiency and light output can be improved using lamp compartment return, but optimum lamp temperatures cannot be achieved under reasonable operating conditions. At the other extreme, a 2-lamp luminaire with $34 \mathrm{~W}$ lamps will always be too cool if lamp compartment retum is used. The implication for design is that each luminaire type has a particular limited range of operating temperatures. The luminaire type 
and HVAC design must be selected so that the optimum lamp temperature can be achieved. Methods for doing this are described in the detailed results.

Another finding is that airflow rate has a limited impact on cooling load profiles, and thus, peak cooling loads due to lighting. Peak cooling loads are primarily dependent on the long term heat storage characteristics of the room, primarily the floor and ceiling slabs. Unless other methods of external heat storage are implemented, such as rock beds or ice storage, or control strategies are used, peak cooling loads cannot be altered to a great extent.

The use of carpet is beneficial because it allows the floor slab to heat up more, thereby storing more heat and slowing down the pickups of cooling load. The plenum area is the best place to store the heat from the lighting system for a number of reasons. First, both the lighting system (heat source) and floor slabs (heat storage) are in contact with the plenum. Second, there may be other structural elements in the plenum for additional heat storage. Third, the plenum can heat up without disturbing the building occupants in the conditioned space.

The effect of furnishings, such as desks, chairs and file cabinets, is to slightly speed up the pickup of cooling load due to lights. The furnishings absorb radiant energy from the lighting system and transfer it to the room air by convection. Although the furnishings add more thermal mass to the room, it is in the conditioned space where it cannot heat up much, thereby allowing little additional heat storage.

\section{References}

1. United States Department of Energy, Overview of Building Energy Use and Report of Analysis - 1985, Office of Building and Community Systems, Washington, DC, October, 1985.

2. Treado, S.J., Experimental Plan for Investigation of Lighting and HVAC Interactions, NBSIR 87-3656, National Bureau of Standards, October, 1987.

3. Treado, S.J., Bean, J.W., The Interaction of Lighting, Heating and Cooling Systems in Buildings - Interim Report, NISTIR 88-3860, National Institute of Standards and Technology, September, 1988.

4. IES Lighting Handbook, Reference Edition, Illuminating Engineering Society of North America, 1984.

5. Mitalas, G.P., Cooling Load Caused by Lights, Transactions CSME Vol. 2, No. 3, 1973-1974.

6. ASHRAE Handbook of Fundamentals, ASHRAE, Atlanta, GA, 1989. 


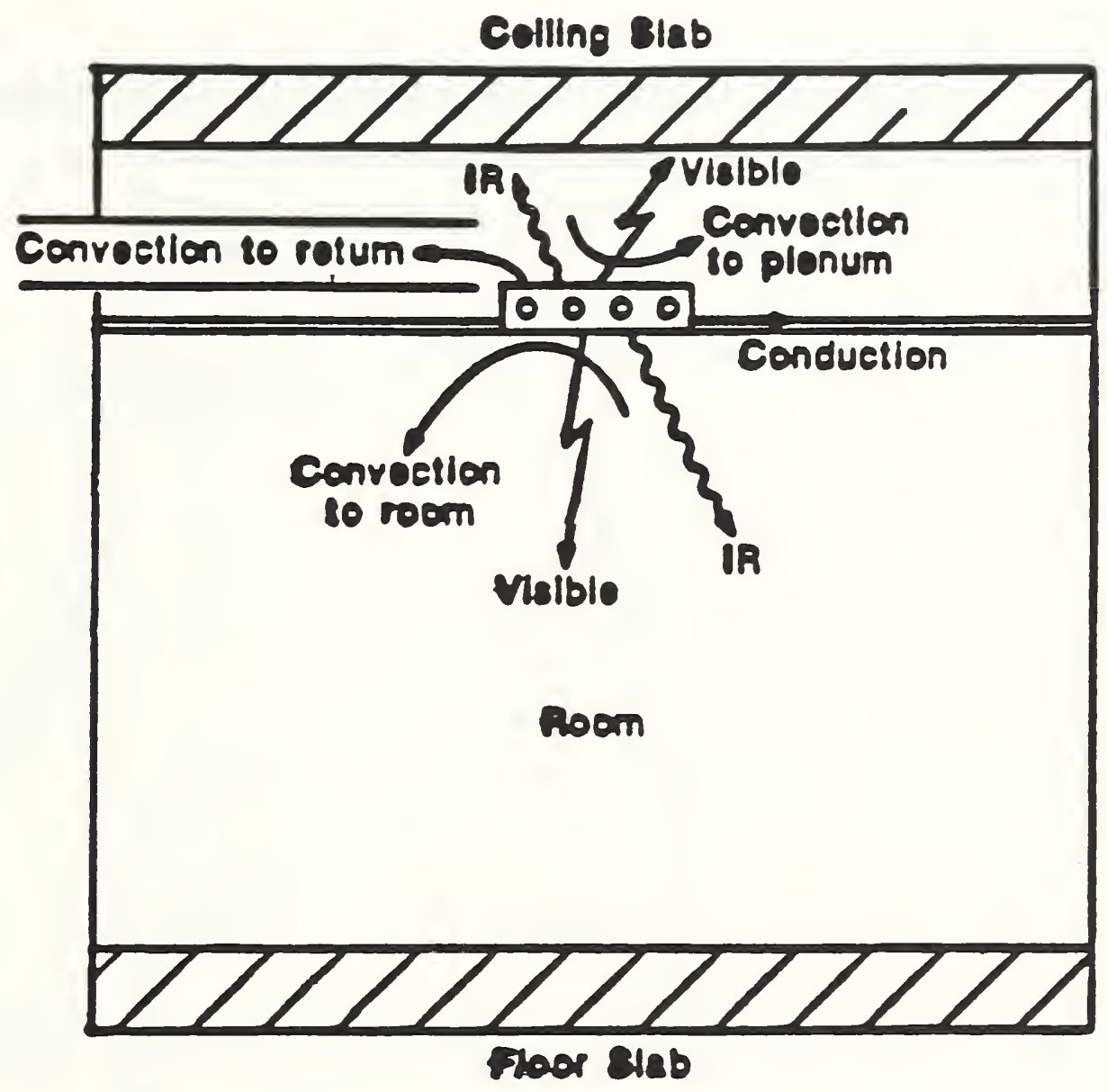

Figure 1. Energy distribution fractions from a typical luminaire 


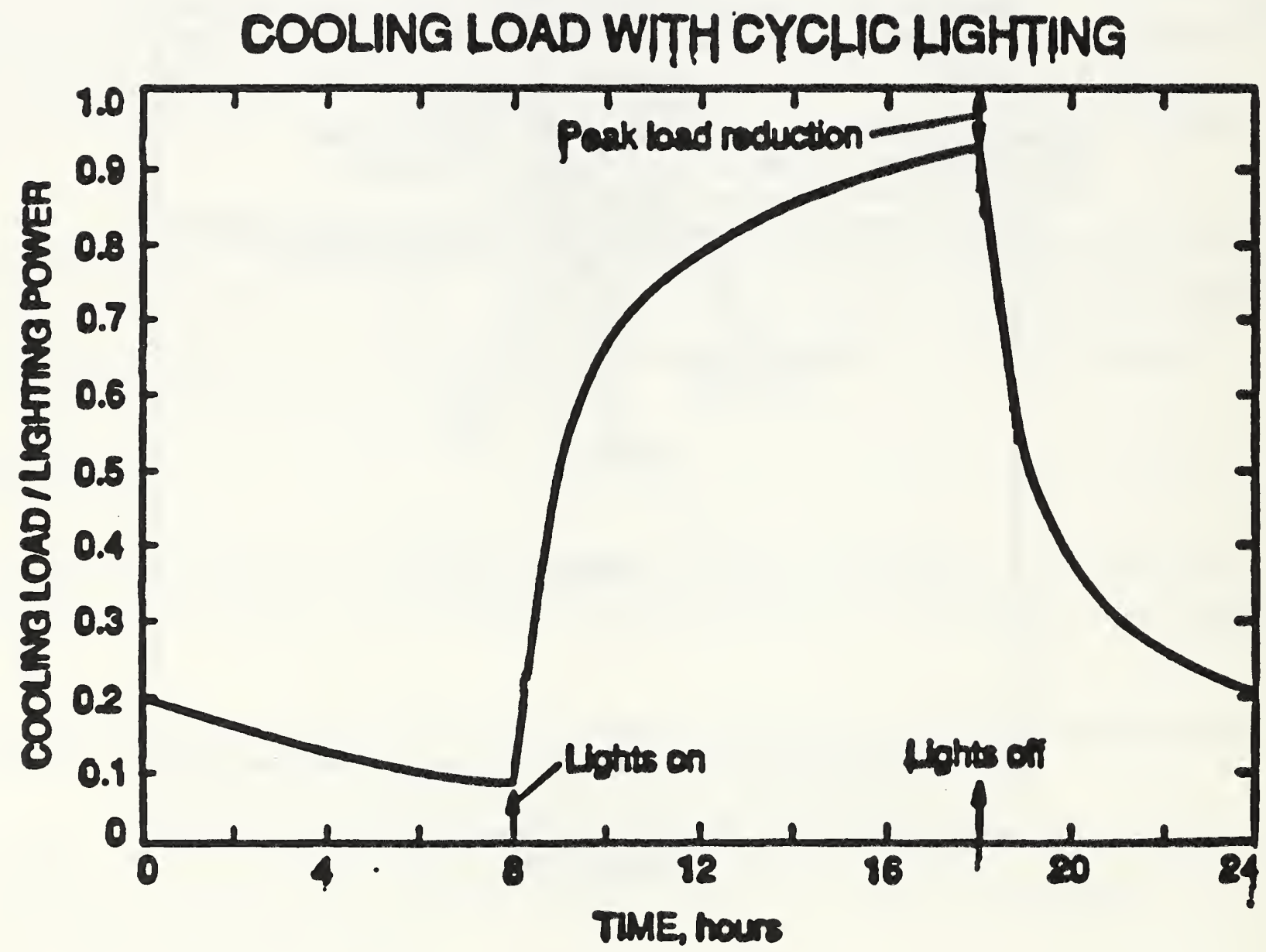

Figure 2. Cooling load profile with cyclic lighting 


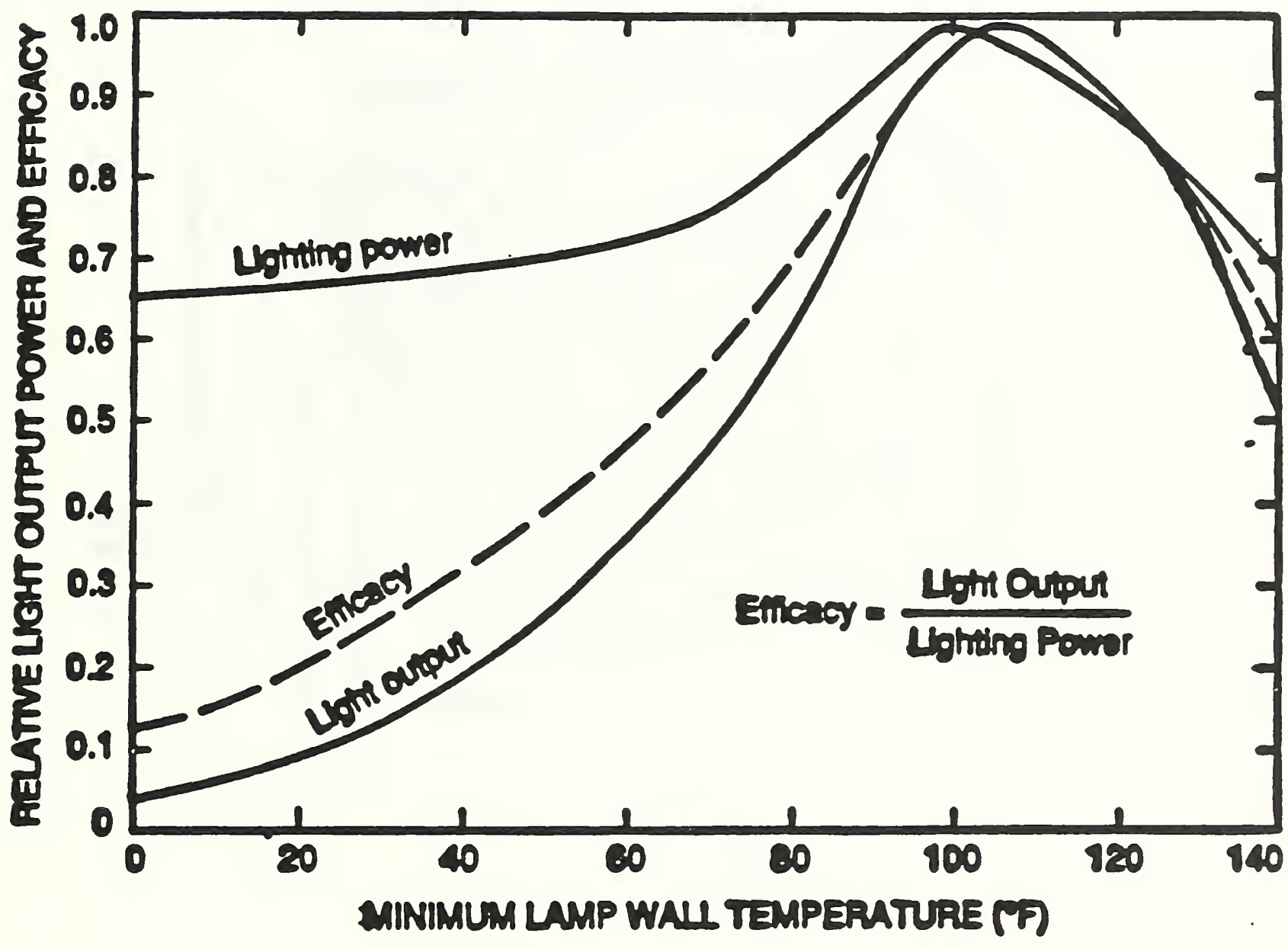

Figure 3. Temperature dependence of fluorescent lamps 


\section{Uohitingarvac Teat Fachinty}

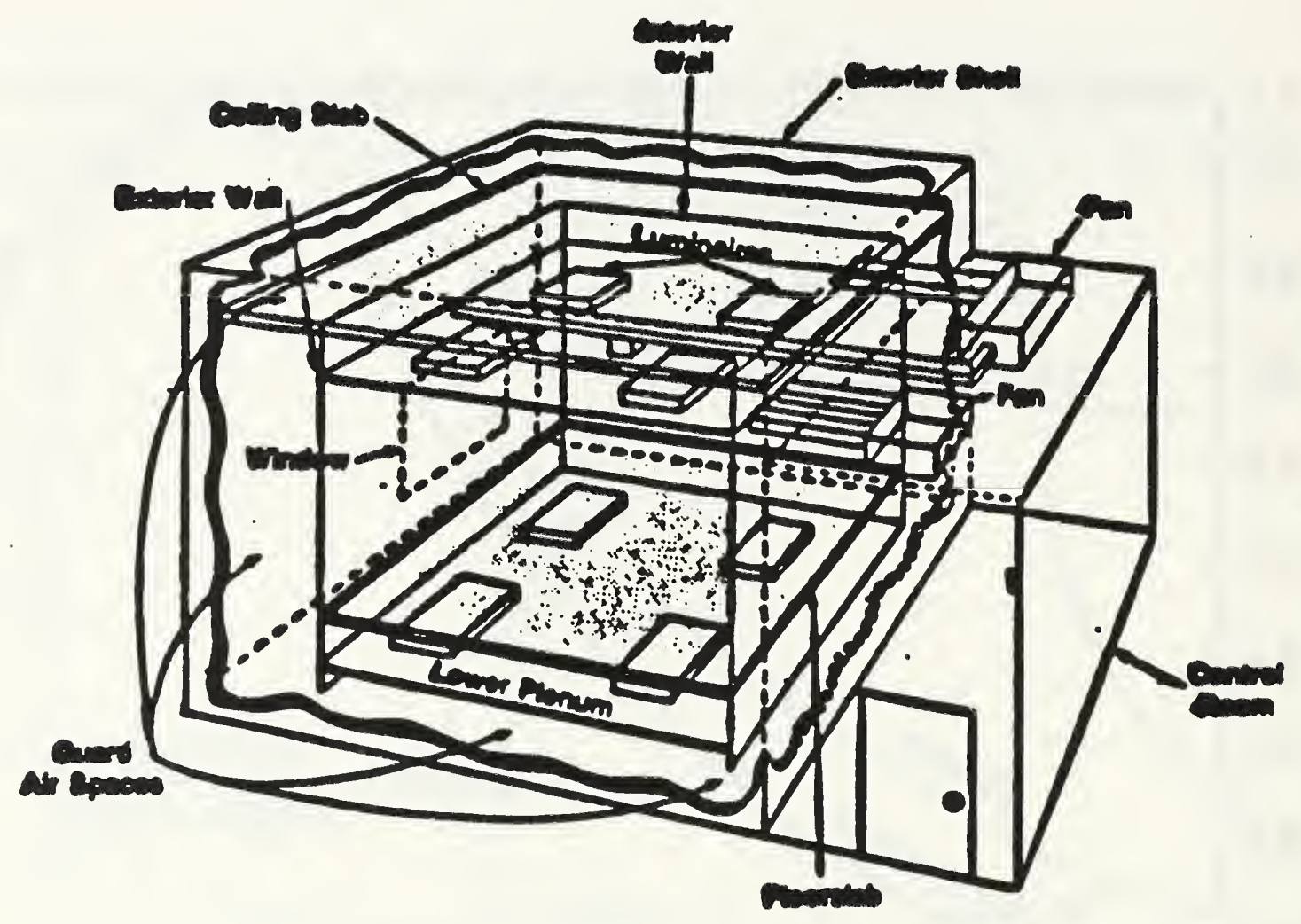

Figure 4. Cut-away schematic view of lighting test facility 


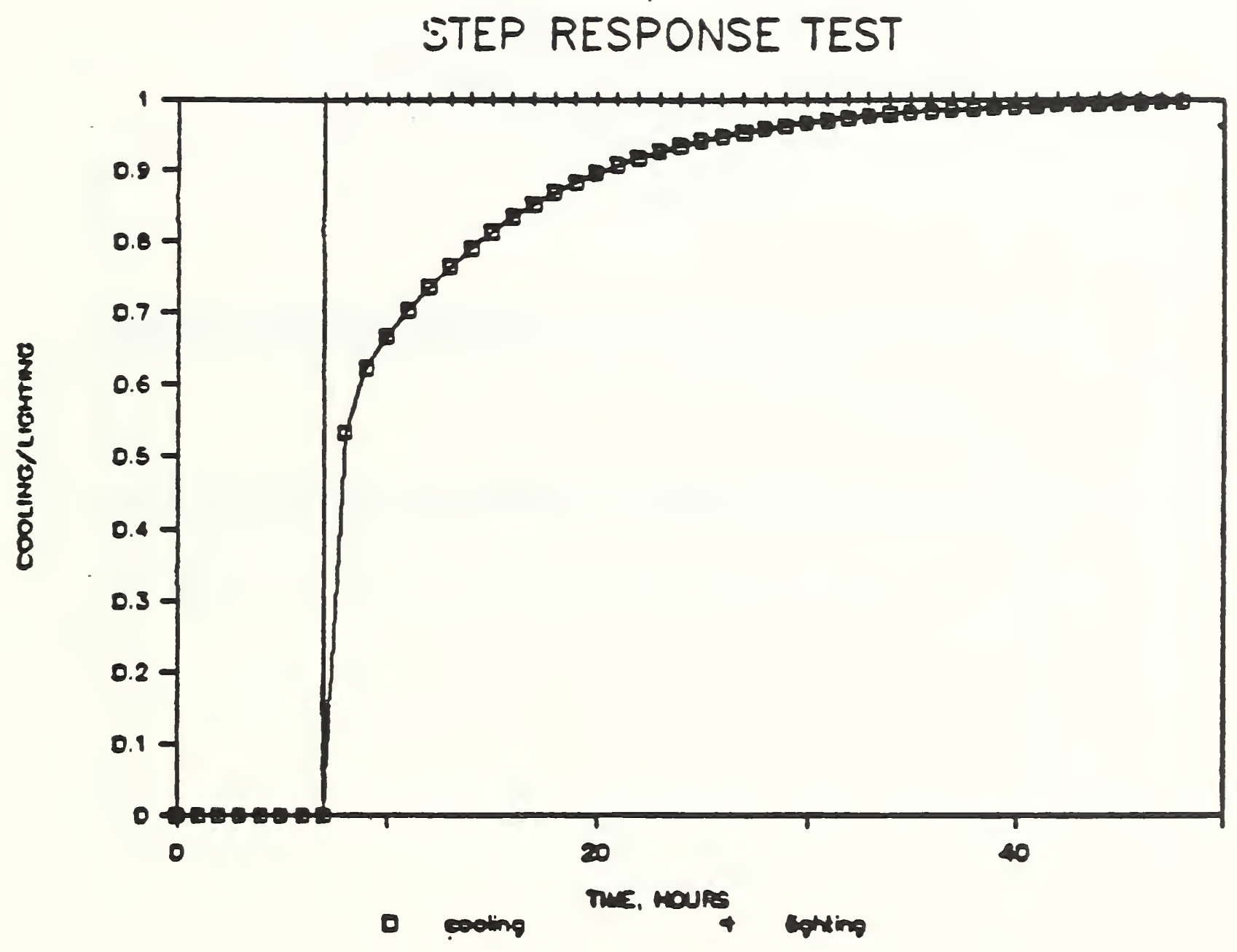

Figure 5. Typical transient test of cooling due to lighting 
ITB, CG, 4X2 ACRL, 40W, CARPET, 75F, 200 CFM COOLING LOAD. LIGHTING POWER (WATTS): ROOM AIR FLOW (CFM)

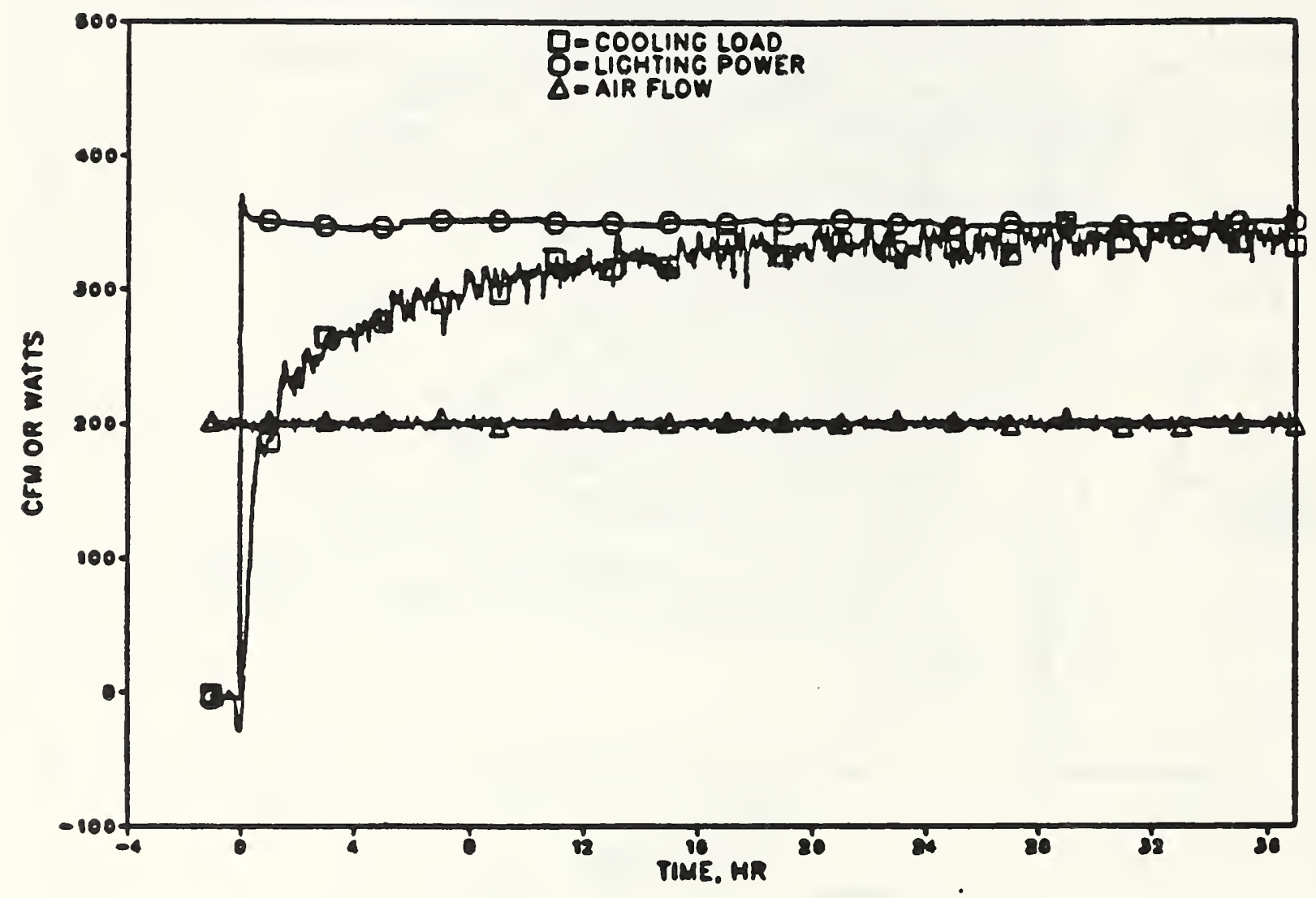

Figure 6. Measured cooling load, lighting power and airflow rate for lights-on test 
ITB, CG, 4X2 ACRL, 40W, CARPET, 75F, 200 CFM ROOMPLENUM AIR TEMPERATURE

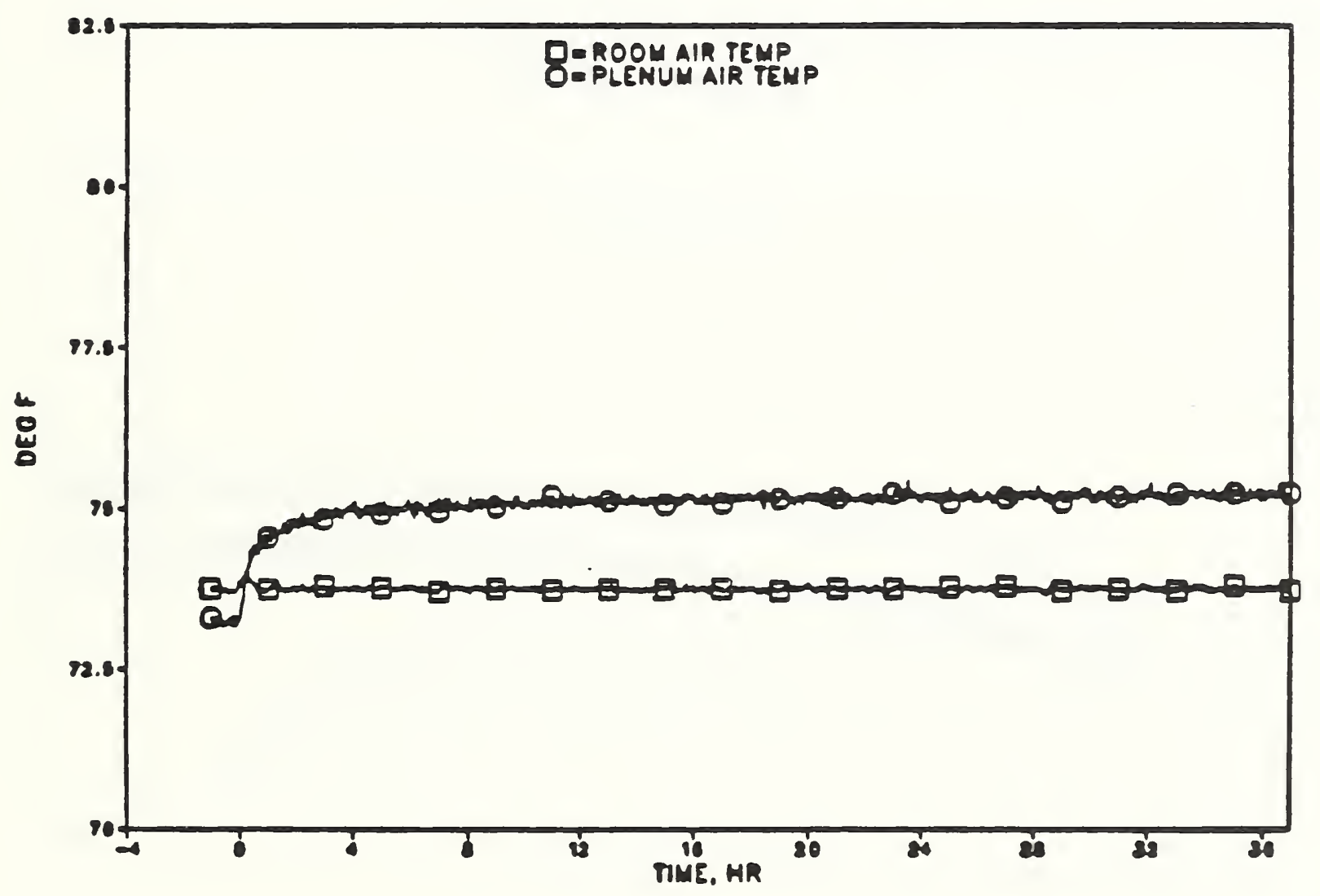

Figure 7. Measured room and plenum air temperatures for lights-on test 
ITB, CG, 4X2 ACRL, 40W, CARPET, 75F, 200 CFM FLOOR/CEILIMG SURFACE TEMPERATURE

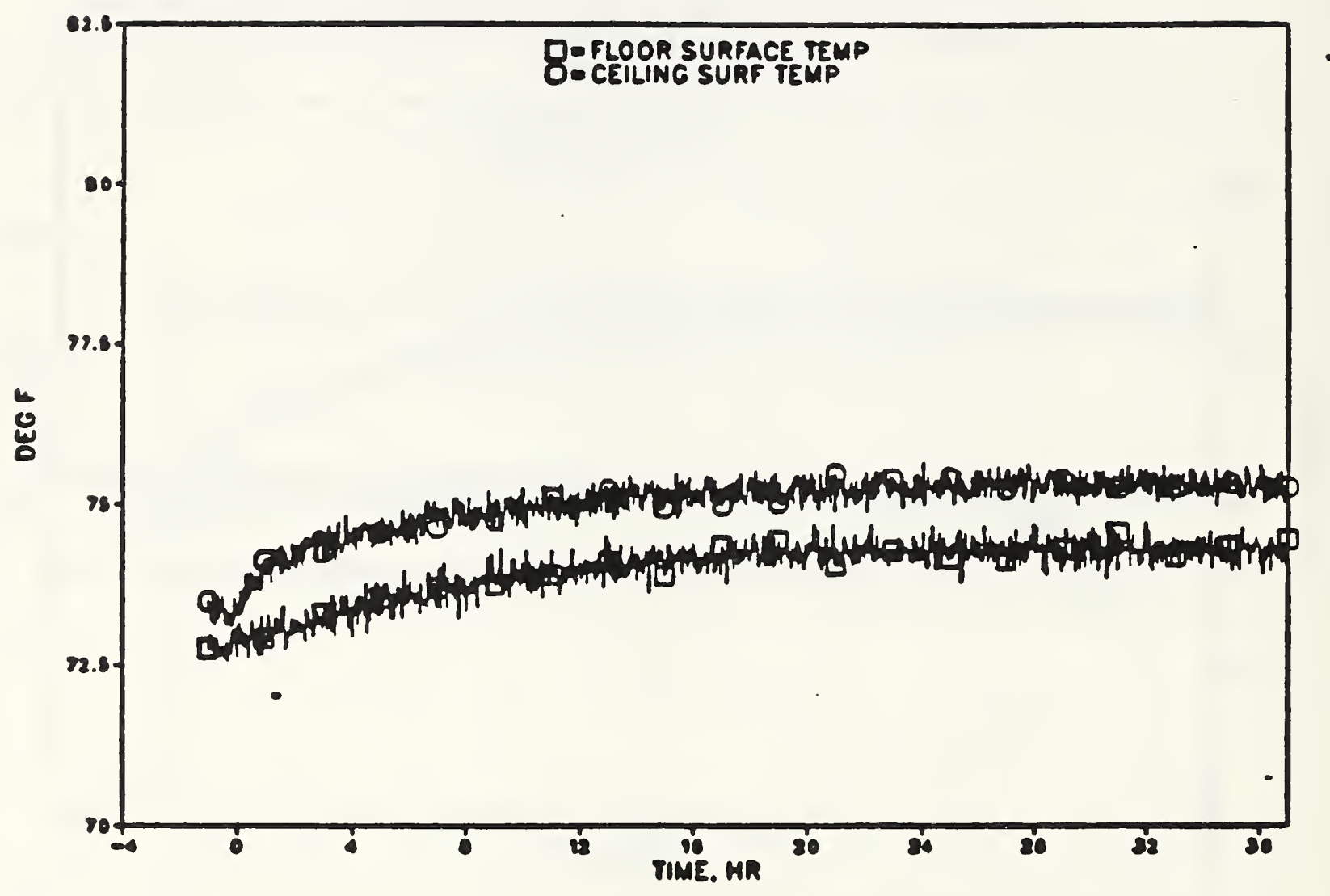

Figure 8. Measured floor and ceiling surface temperatures for lights-on test 
ITB, CG, 4X2 ACRL, 40W, CARPET, 75F, 200 CFM ROOMPLENUM WALL SURFACE TEMPERATURE

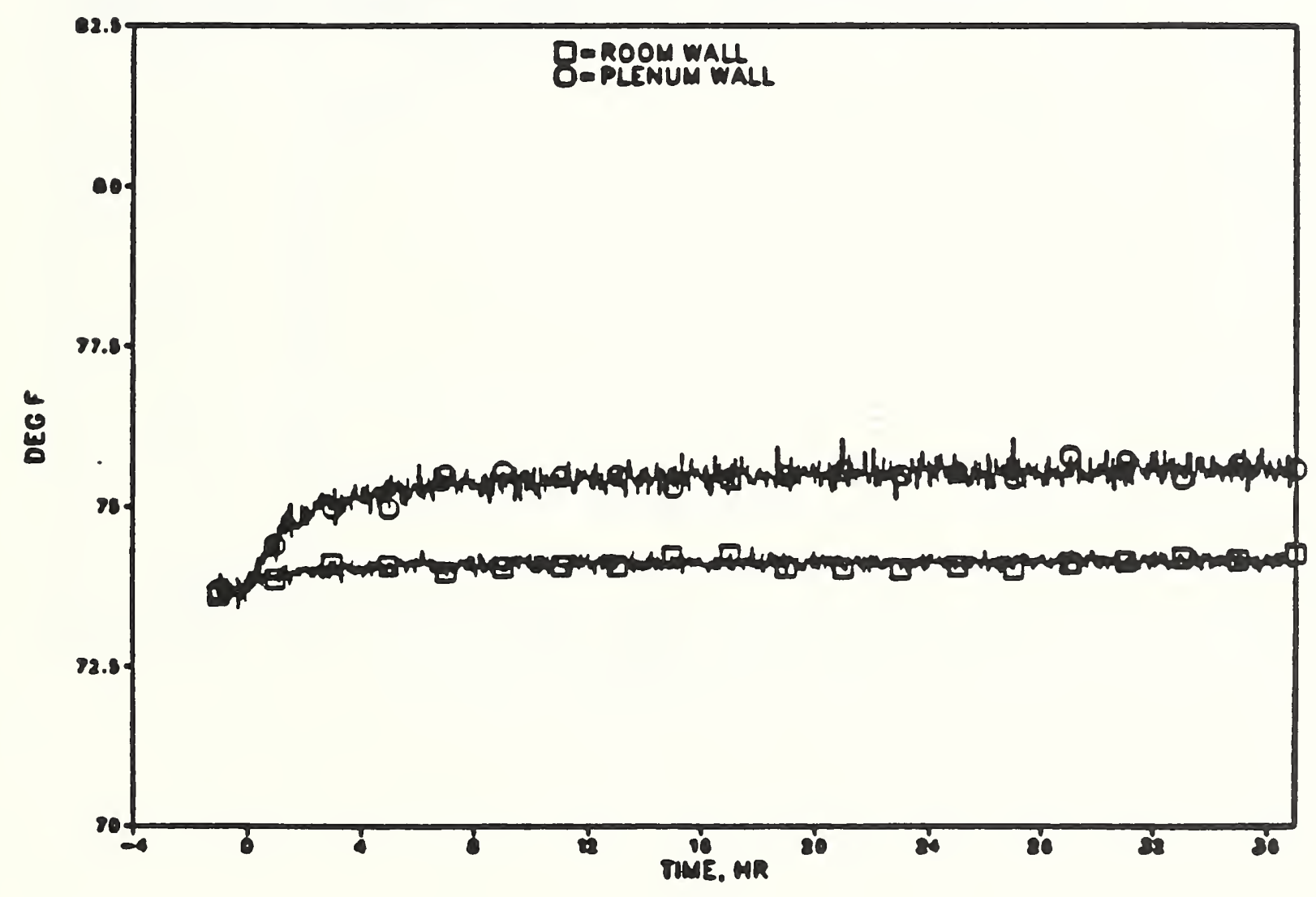

Figure 9. Measured room and plenum wall temperatures for lights-on test 
1TB, CG, 4X2 ACRL, 40W, CARPET, 75F, 200 CFM MINIMUM LAMP WALL SURFACE TEMPERATURE

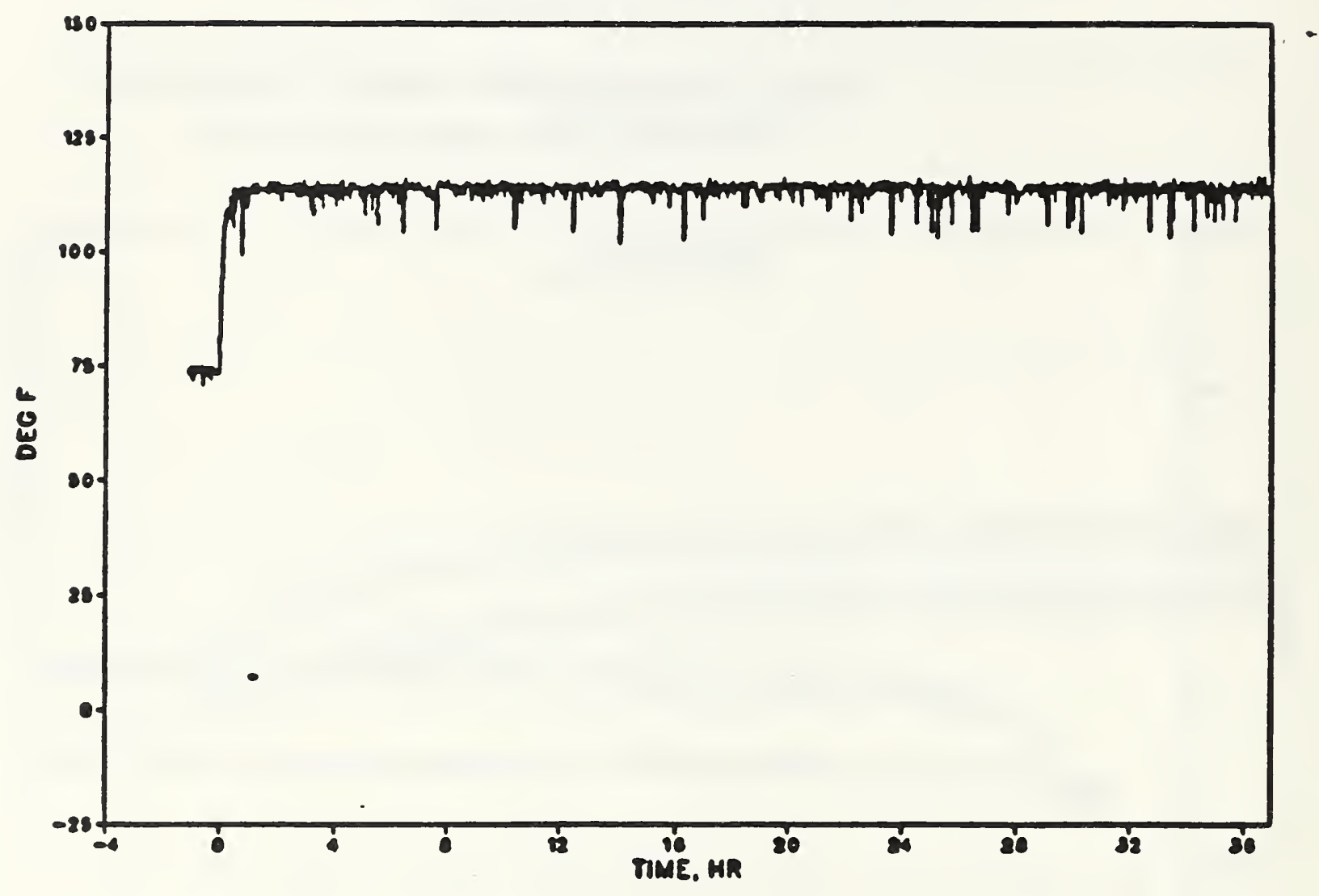

Figure 10. Measured lamp temperatures for lights-on test 


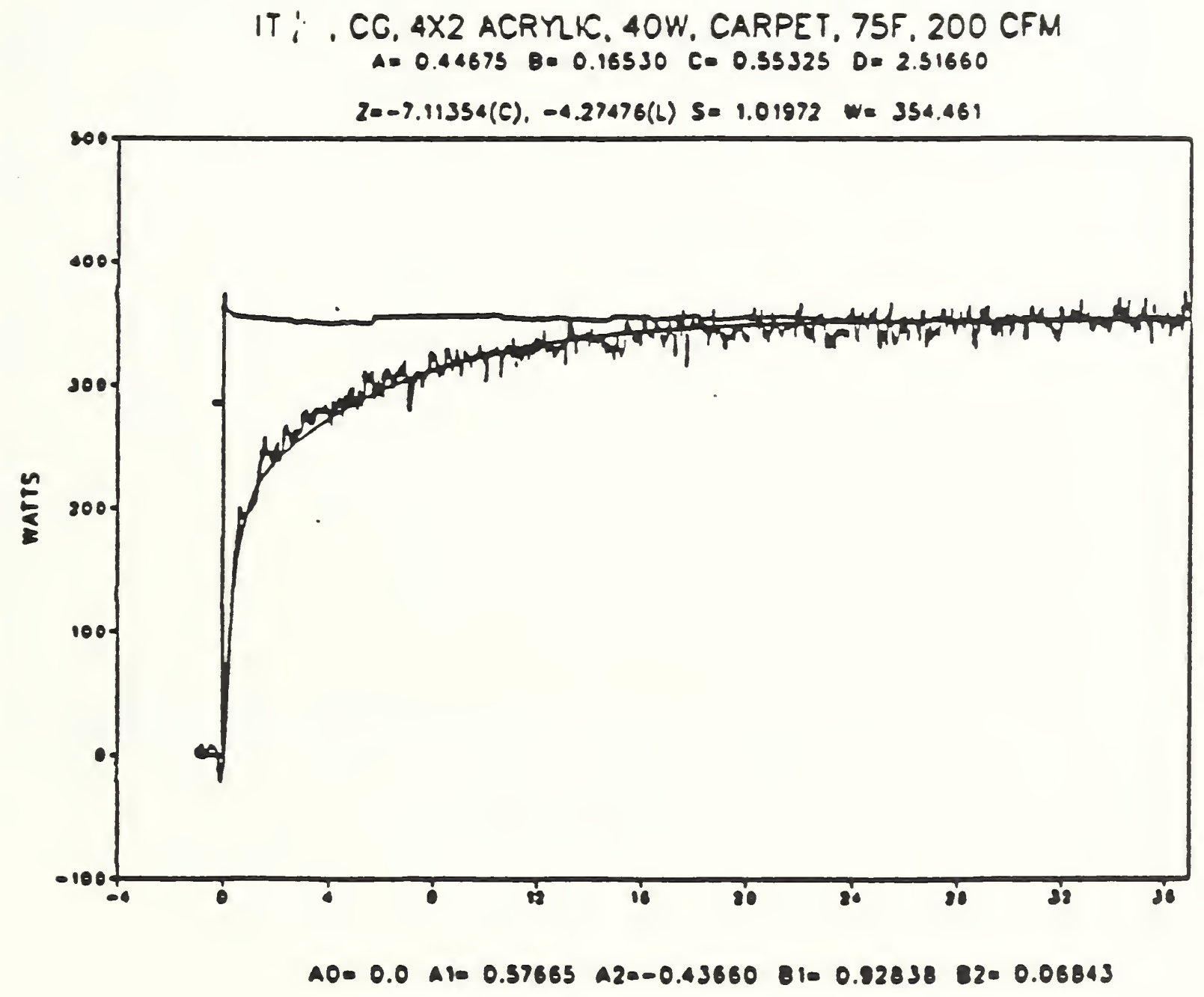

Figure 11. Double exponential curve fit to measured cooling load due to lighting 


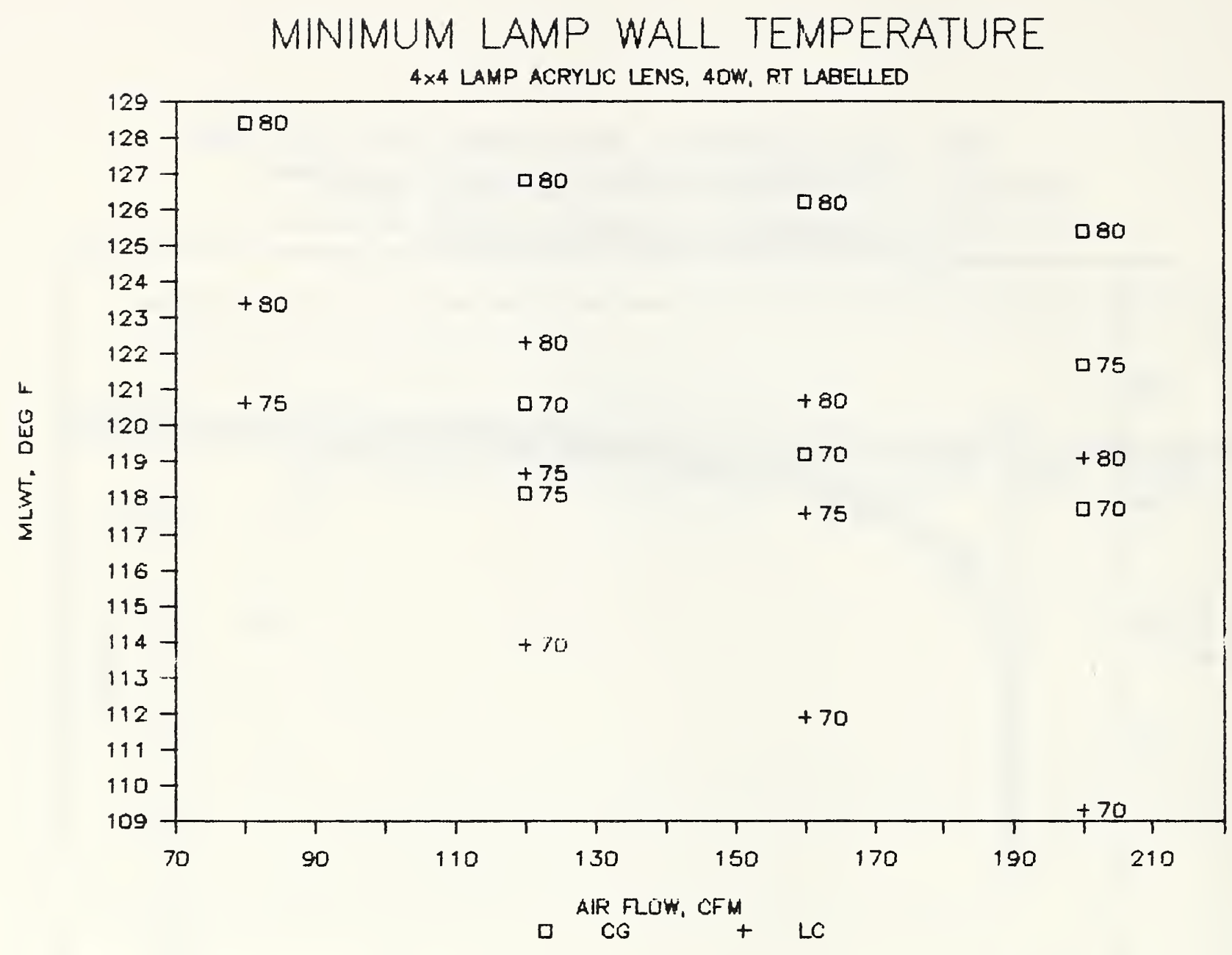

Figure 12. Minimum lamp wall temperatures for the four lamp acrylic lens luminaire 


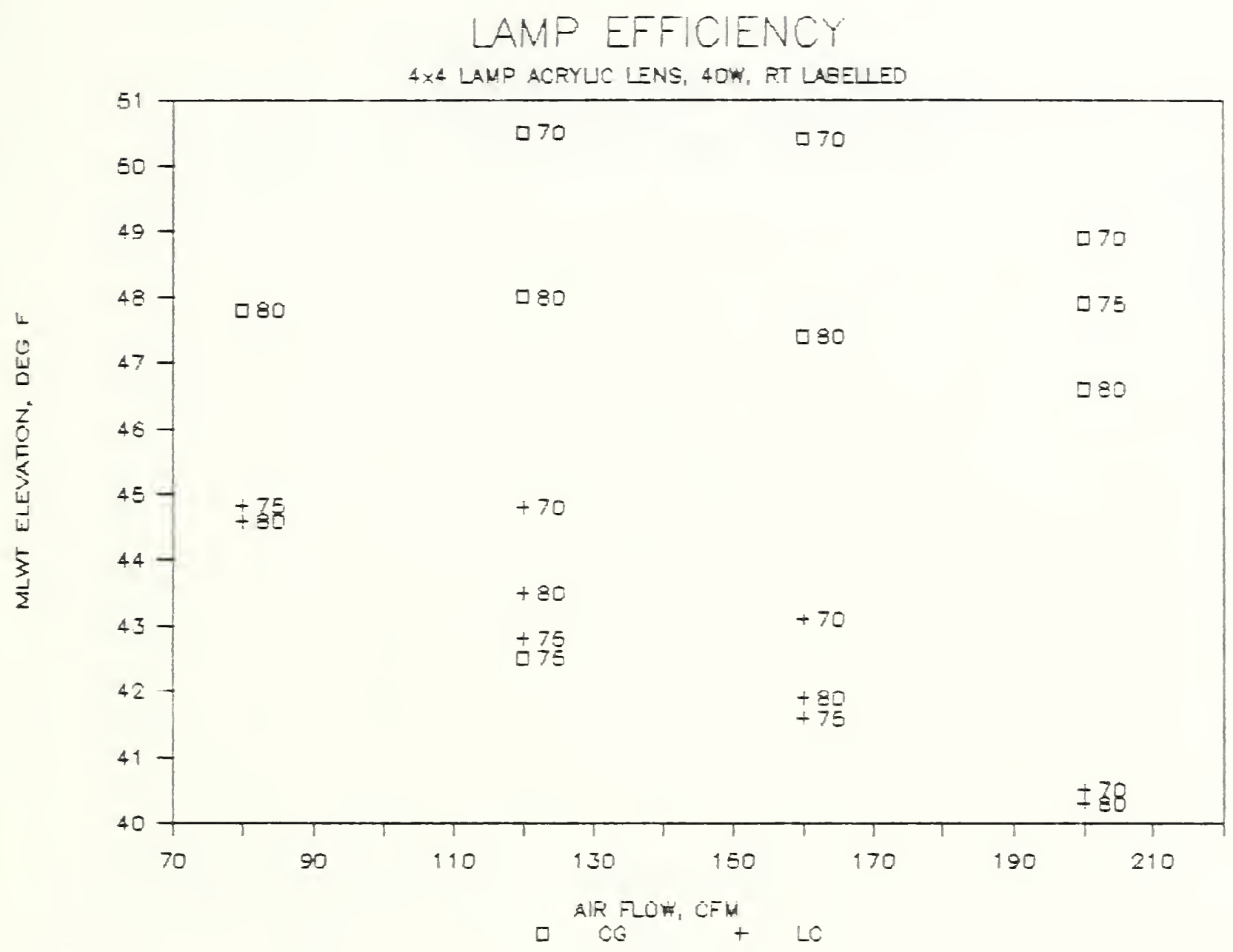

Figure 13. Elevation of minimum lamp wall temperature above room air temperature for the four lamp acrylic lens luminaire 


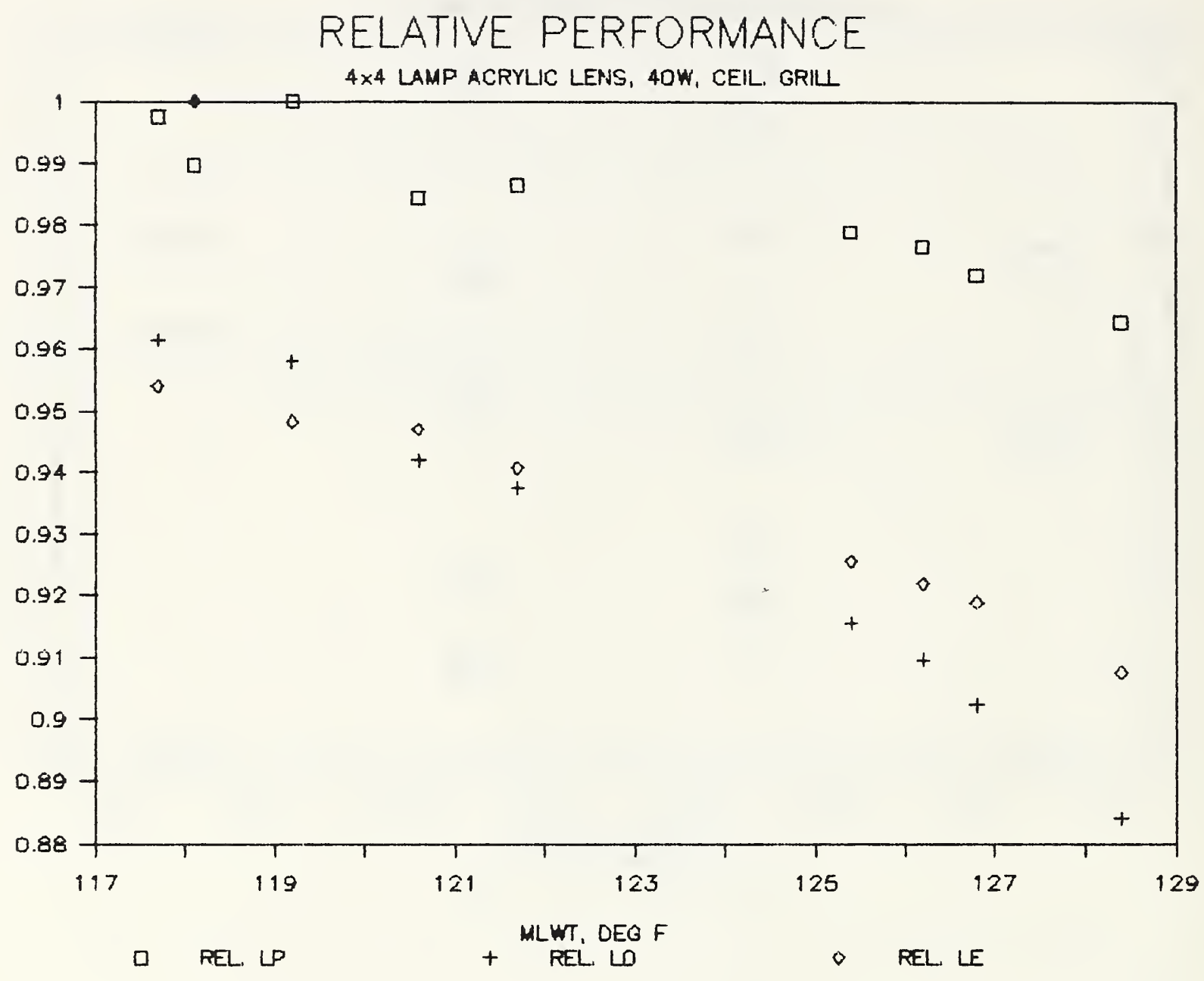

Figure 14. Lighting power, light output and luminous efficacy for the four lamp acrylic lens luminaire with ceiling grill return 


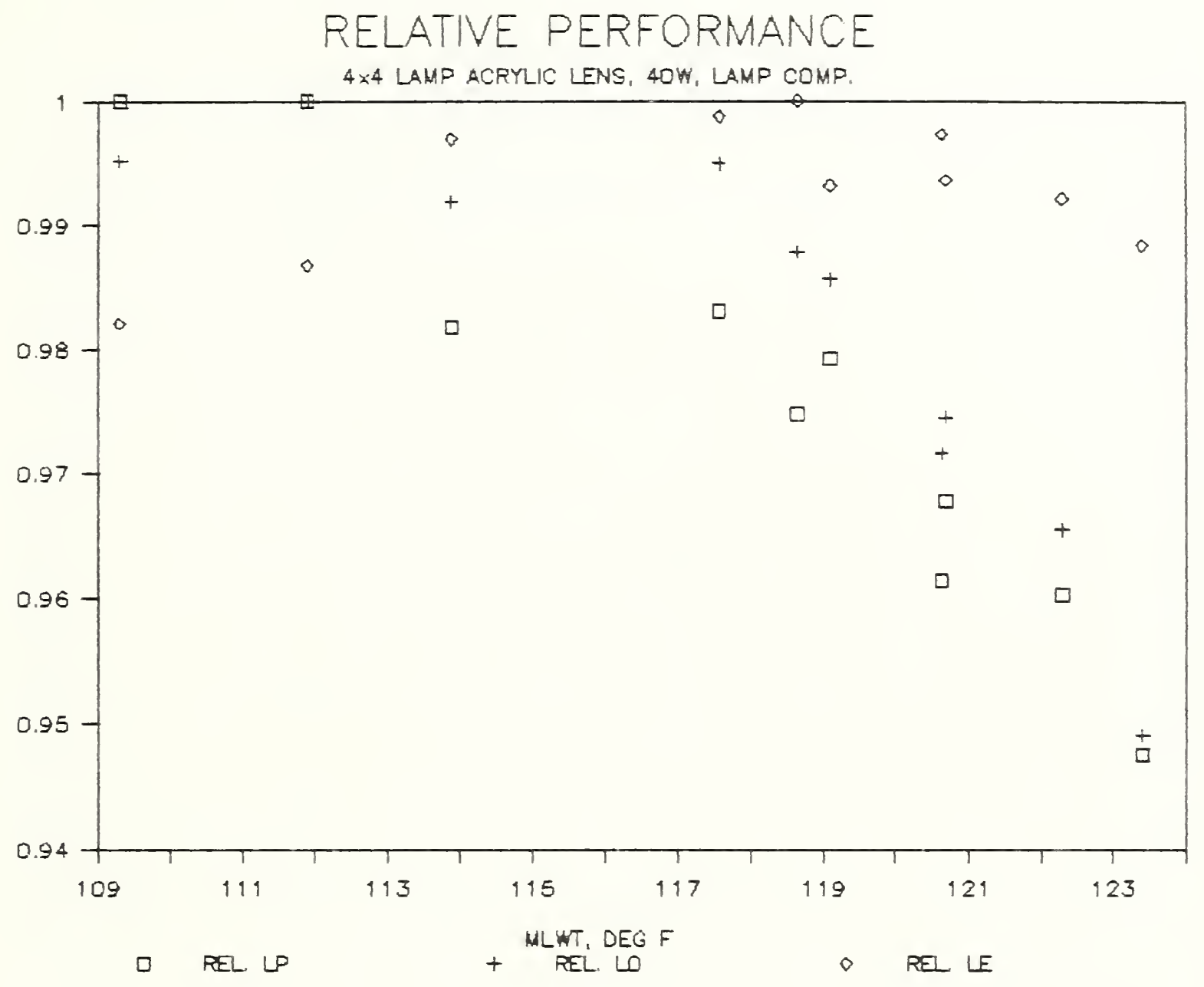

Figure 15. Lighting power, light output and luminous efficacy for the four lampacrylic lens luminaire with lamp compartment return 


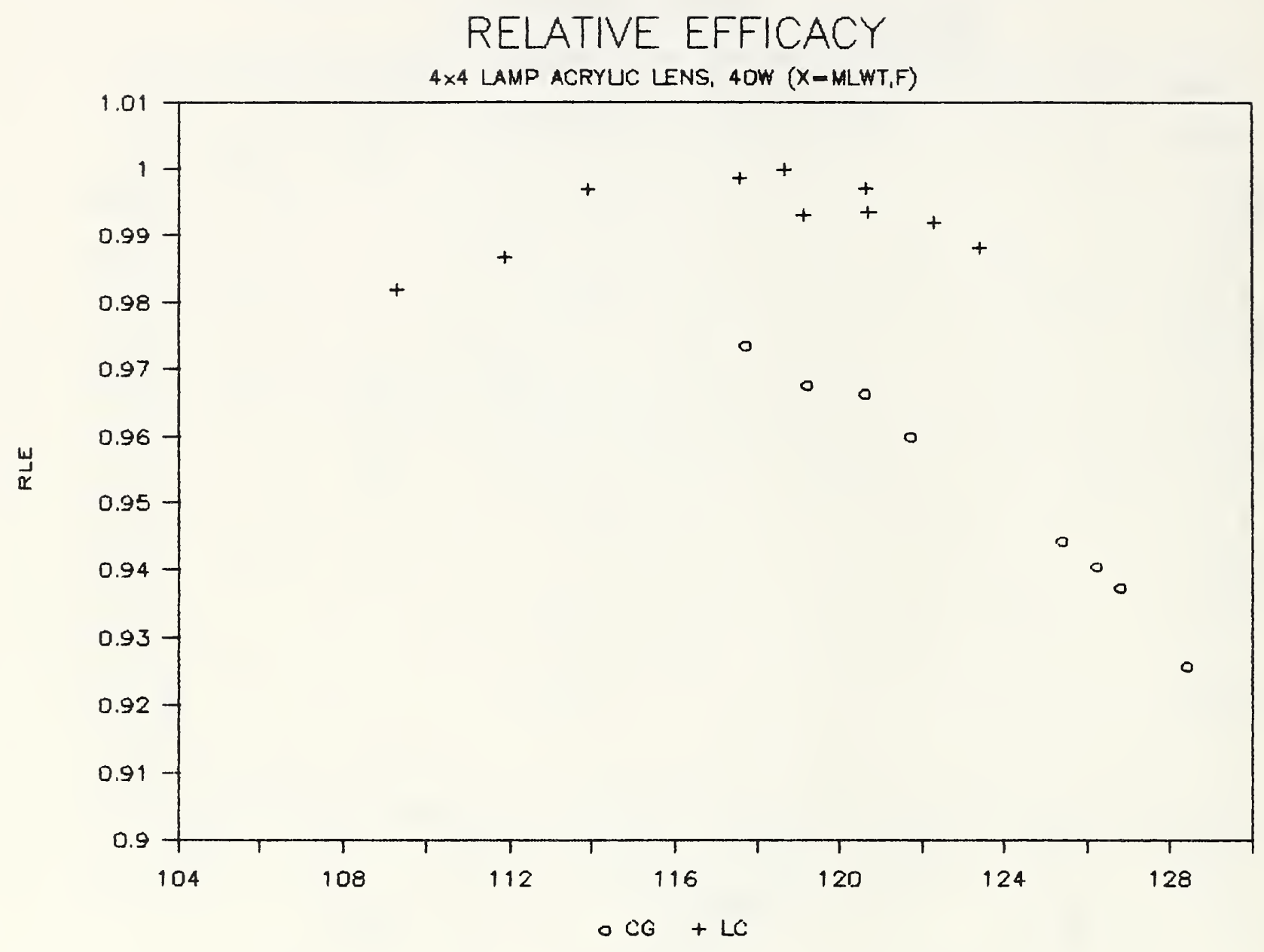

Figure 16. Luminous efficacy for the four lamp acrylic lens luminaire with ceiling grill or lamp compartment return 


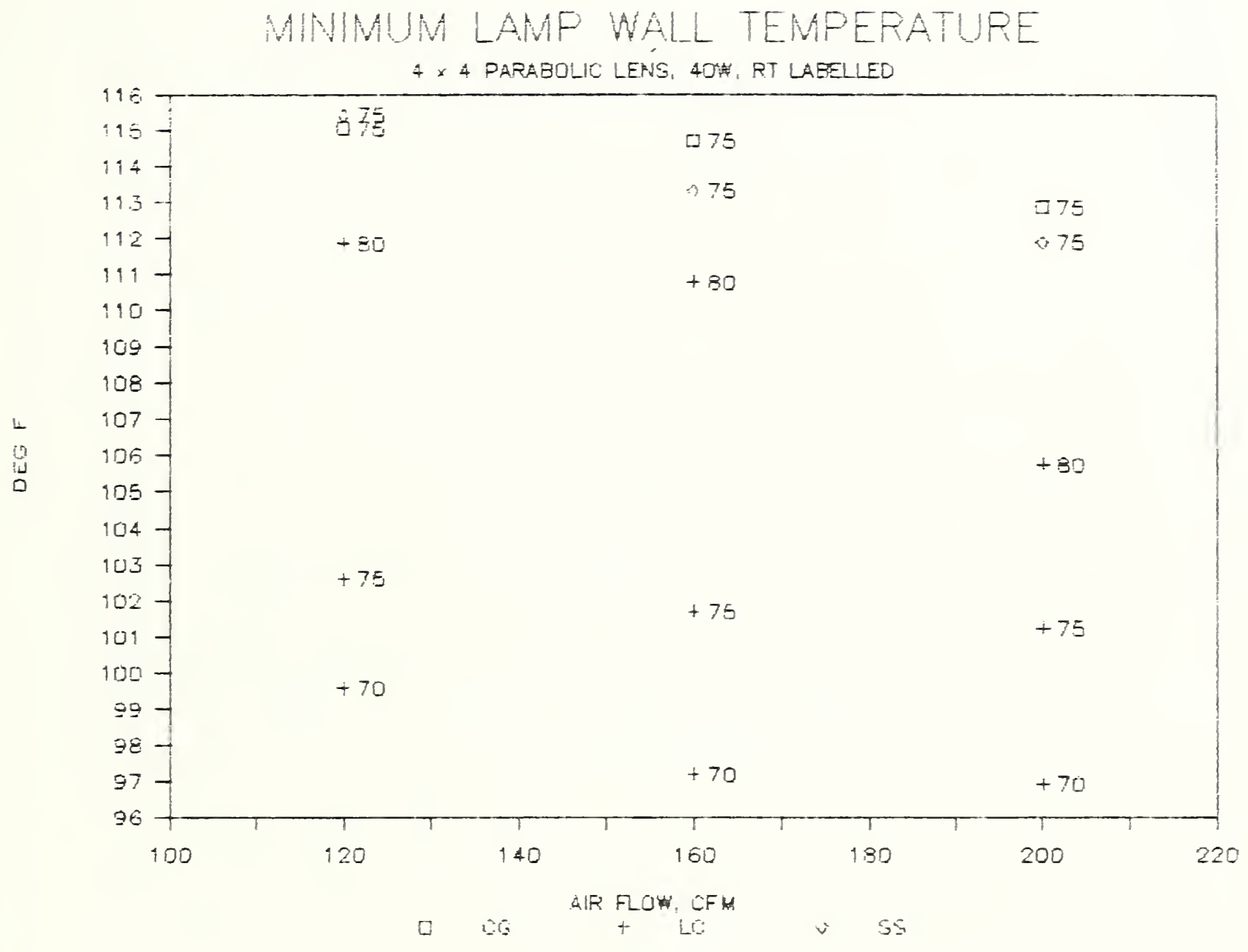

Figure 17. Minimum lamp wall temperatures for the four lamp parabolic diffuser luminaire 


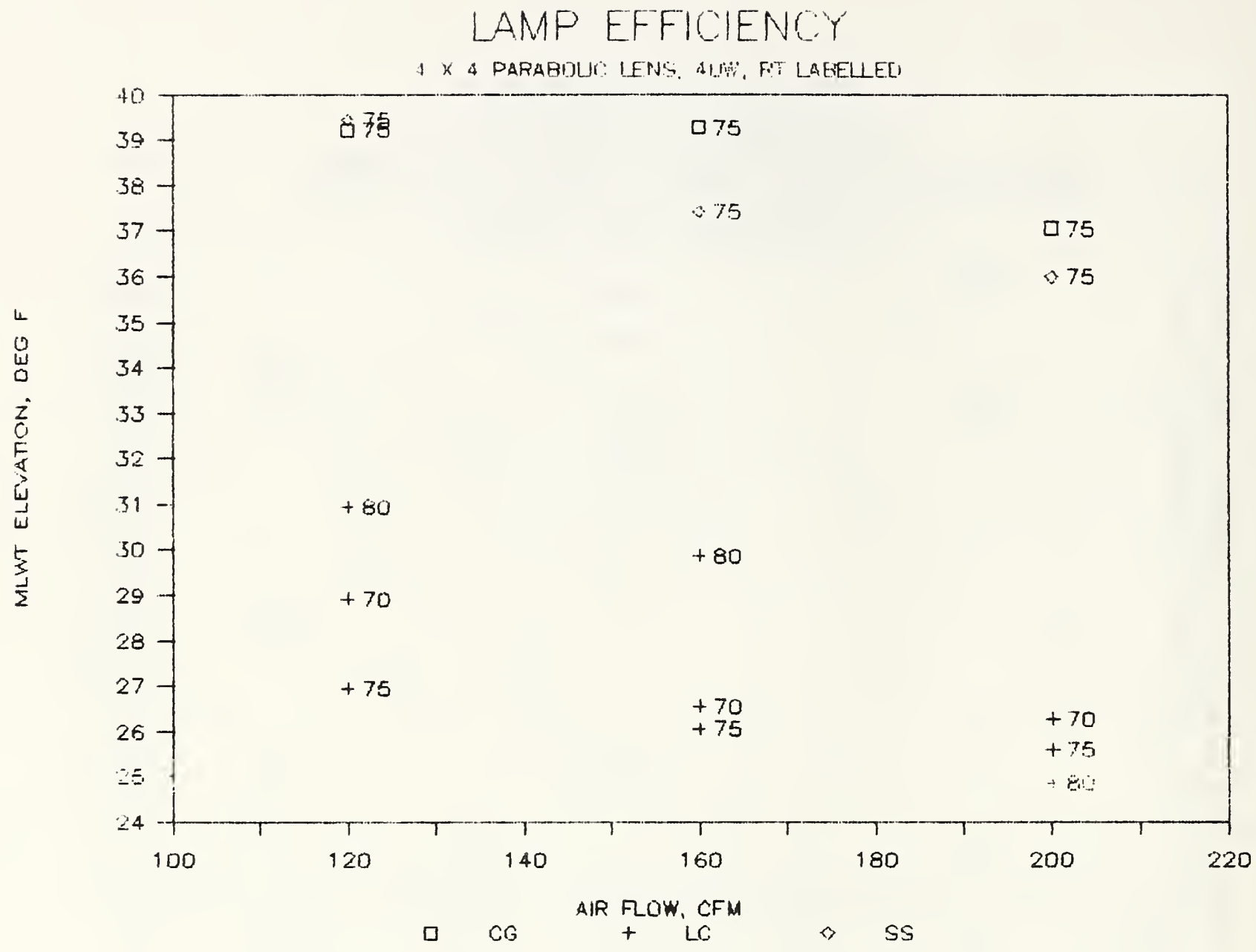

Figure 18. Elevation of minimum lamp wall temperature above room air temperature for the four lamp parabolic diffuser luminaire 


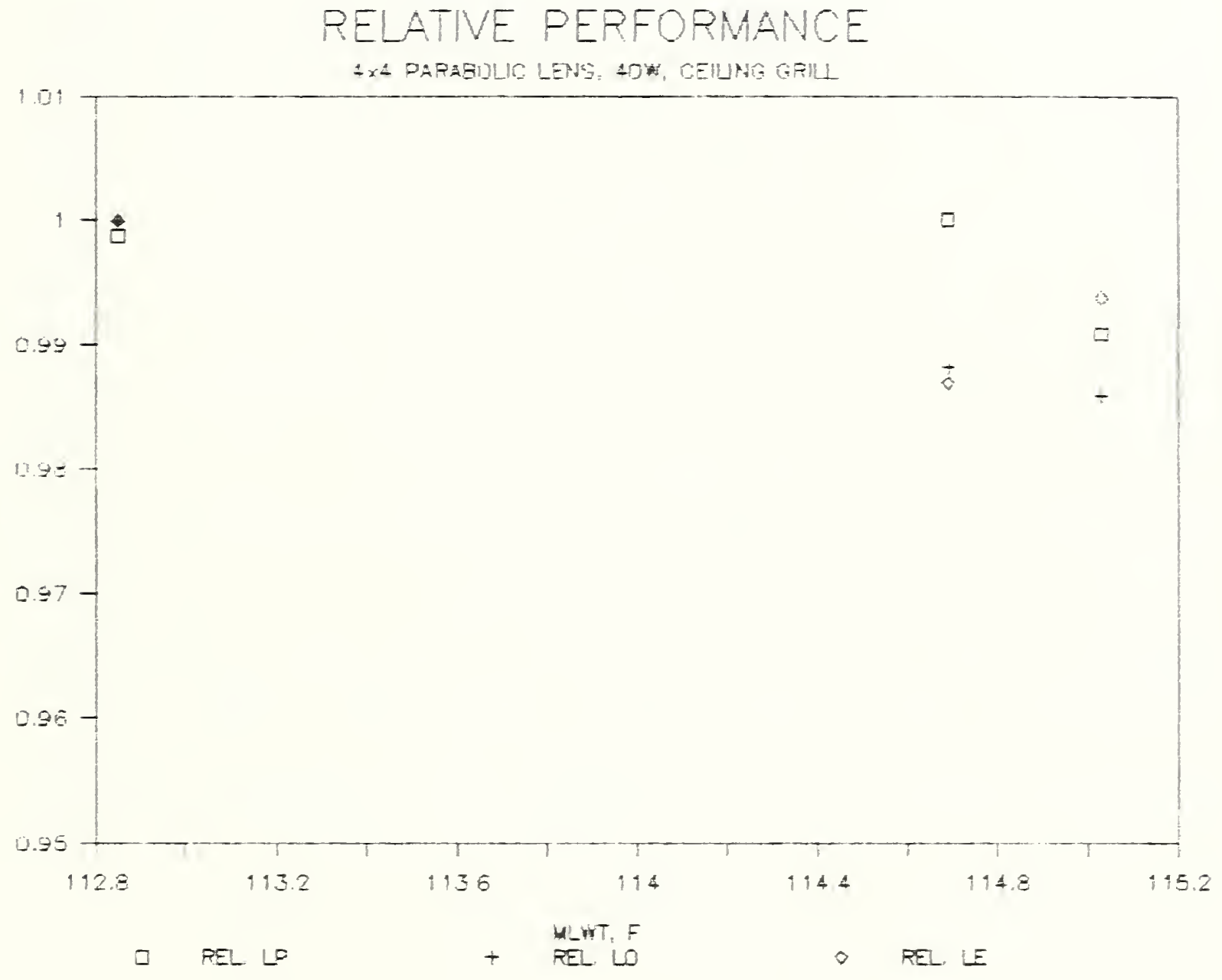

Figure 19. Lighting power, light output and luminous efficacy for the four lamp parabolic diffuser luminaire with ceiling grill retum 
RELATIVE PERFORMANCE

4×4 PARABOLIC LENS, 4OW, SIDE SLDTS

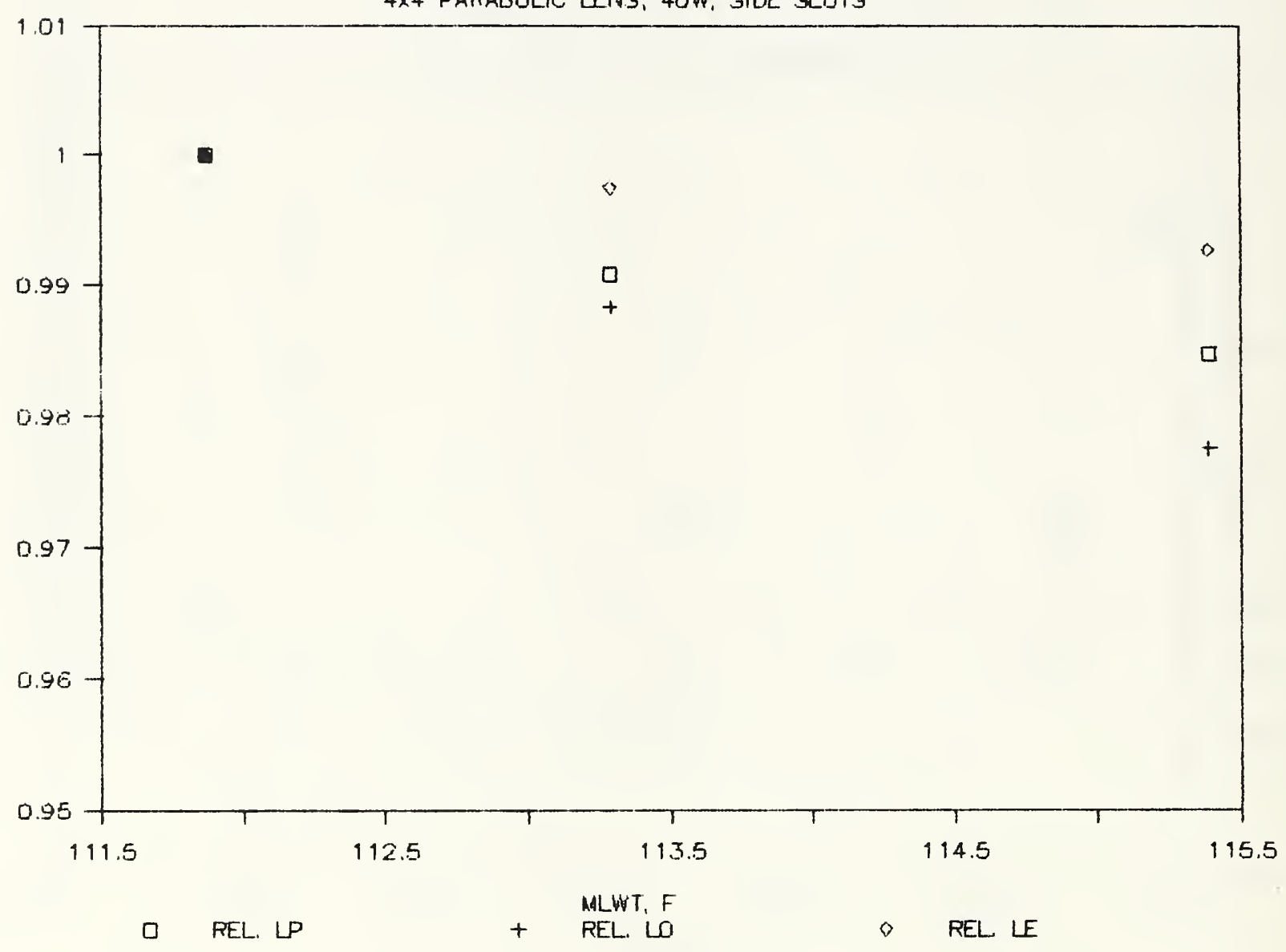

Figure 20. Lighting power, light output and luminous efficacy with side slot return 
RELATIVE PERFORMANCE $4 \times 4$ PARABOUC LENG, 4 DW, LAMP SOMP.

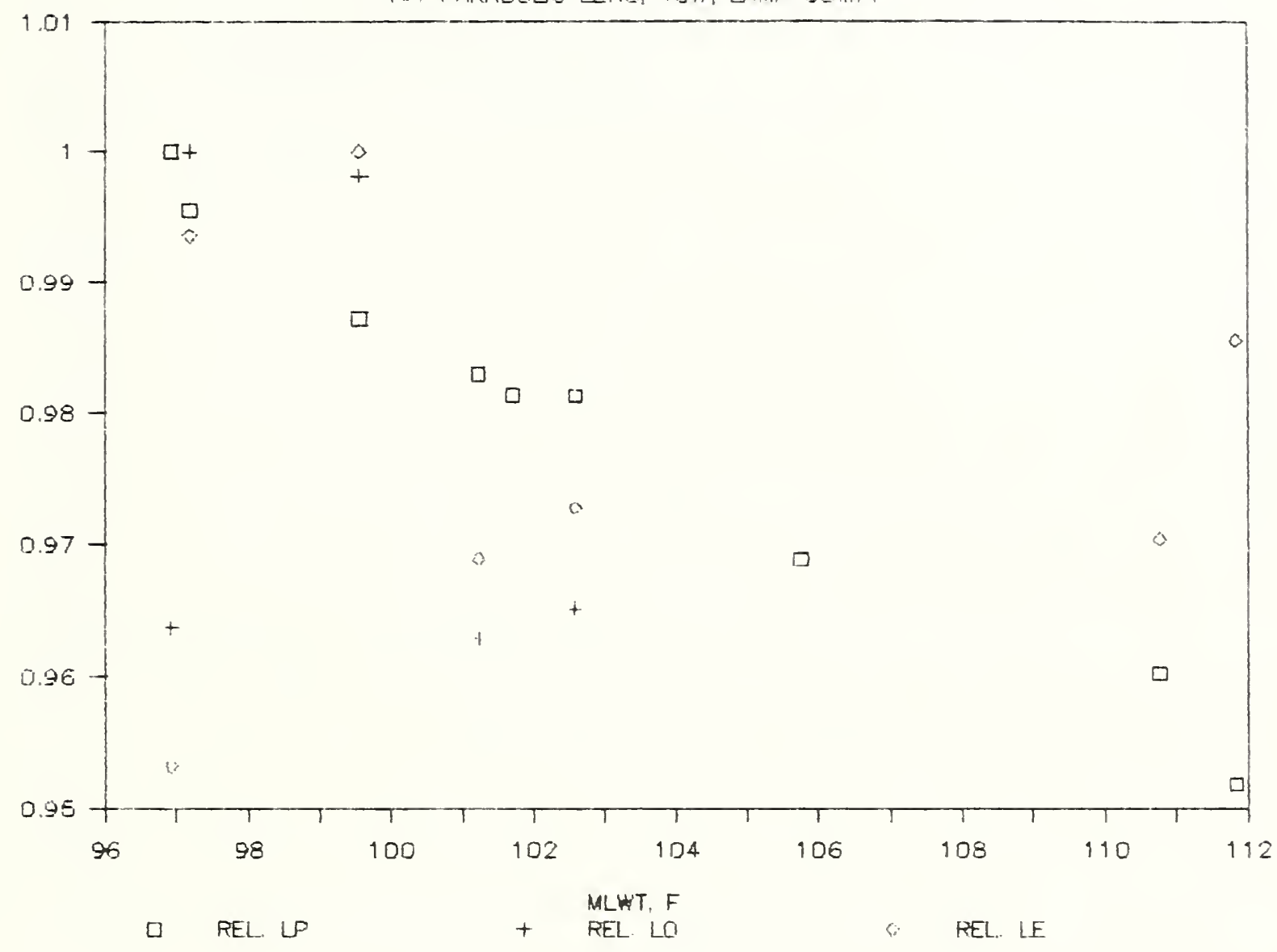

Figure 21. Lighting power, light output and luminous efficacy for the four lamp parabolic diffuser luminaire with lamp compartment return 
1.4 PARABIIIC I.ENS. ATW

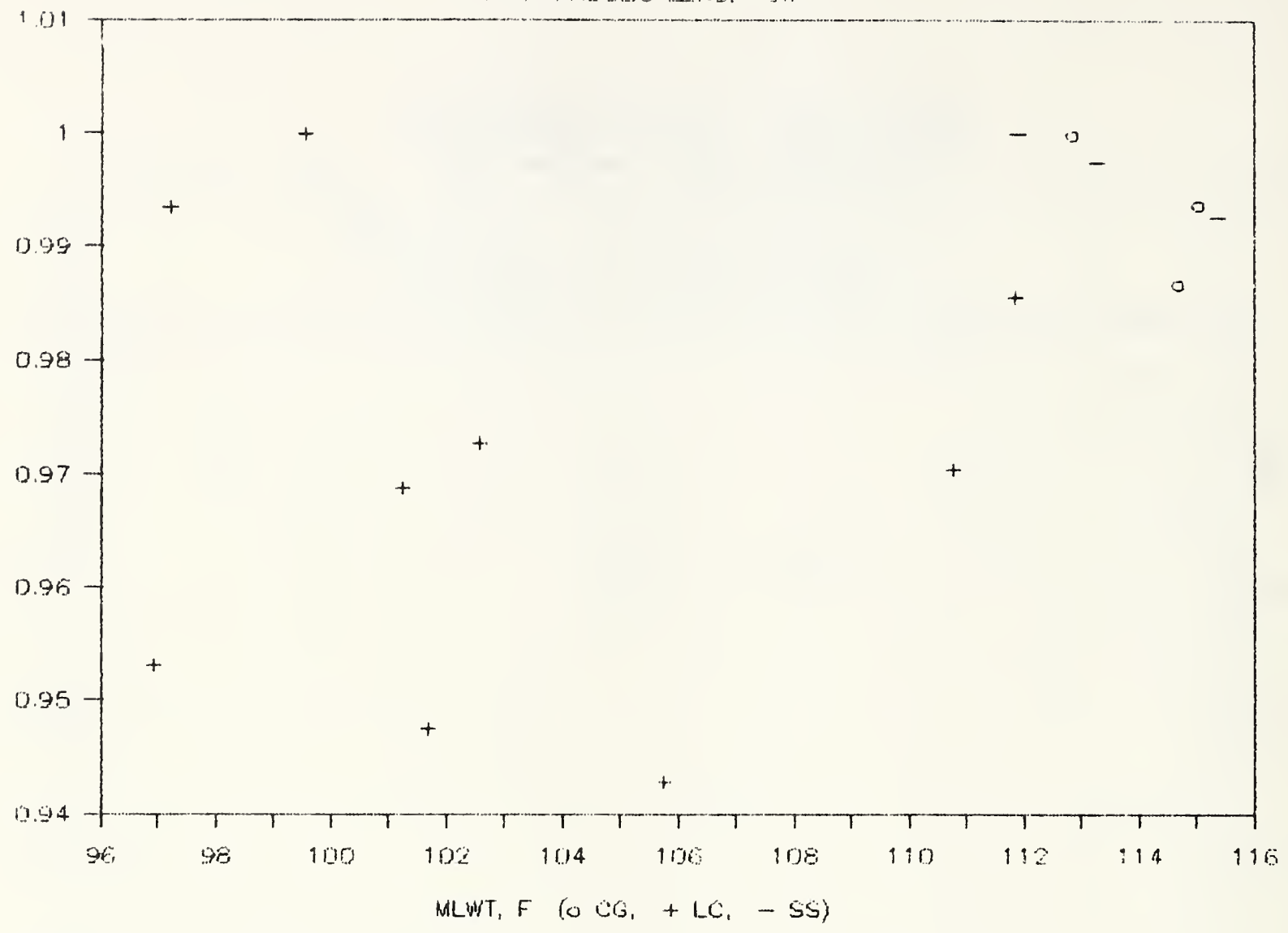

Figure 22. Luminous efficacy for the four lamp parabolic diffuser luminaire with ceiling grill, side slots or lamp compartment return 


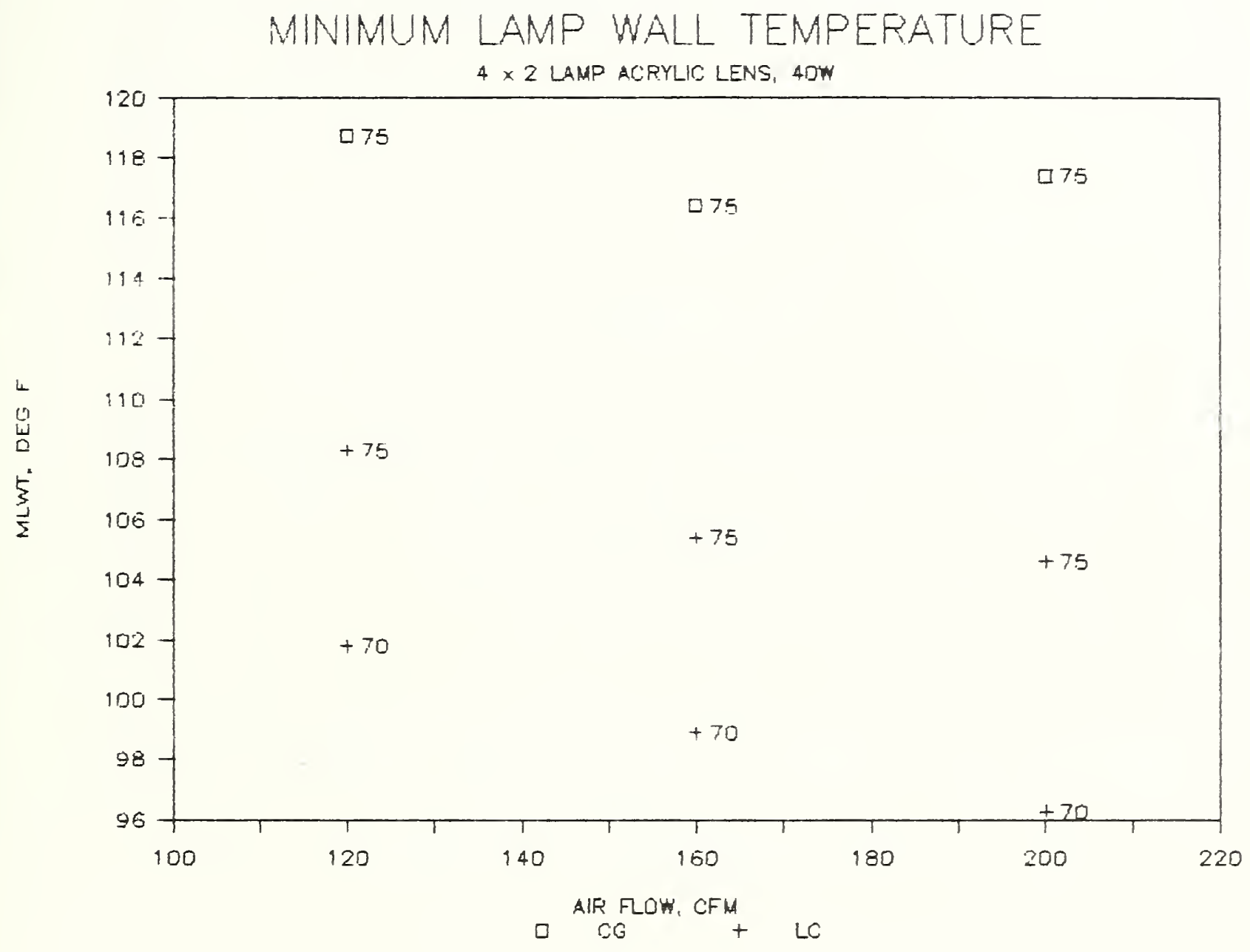

Figure 23. Minimum lamp wall temperature for four two-lamp acrylic lens luminaires with ceiling grill or lamp compartment return 


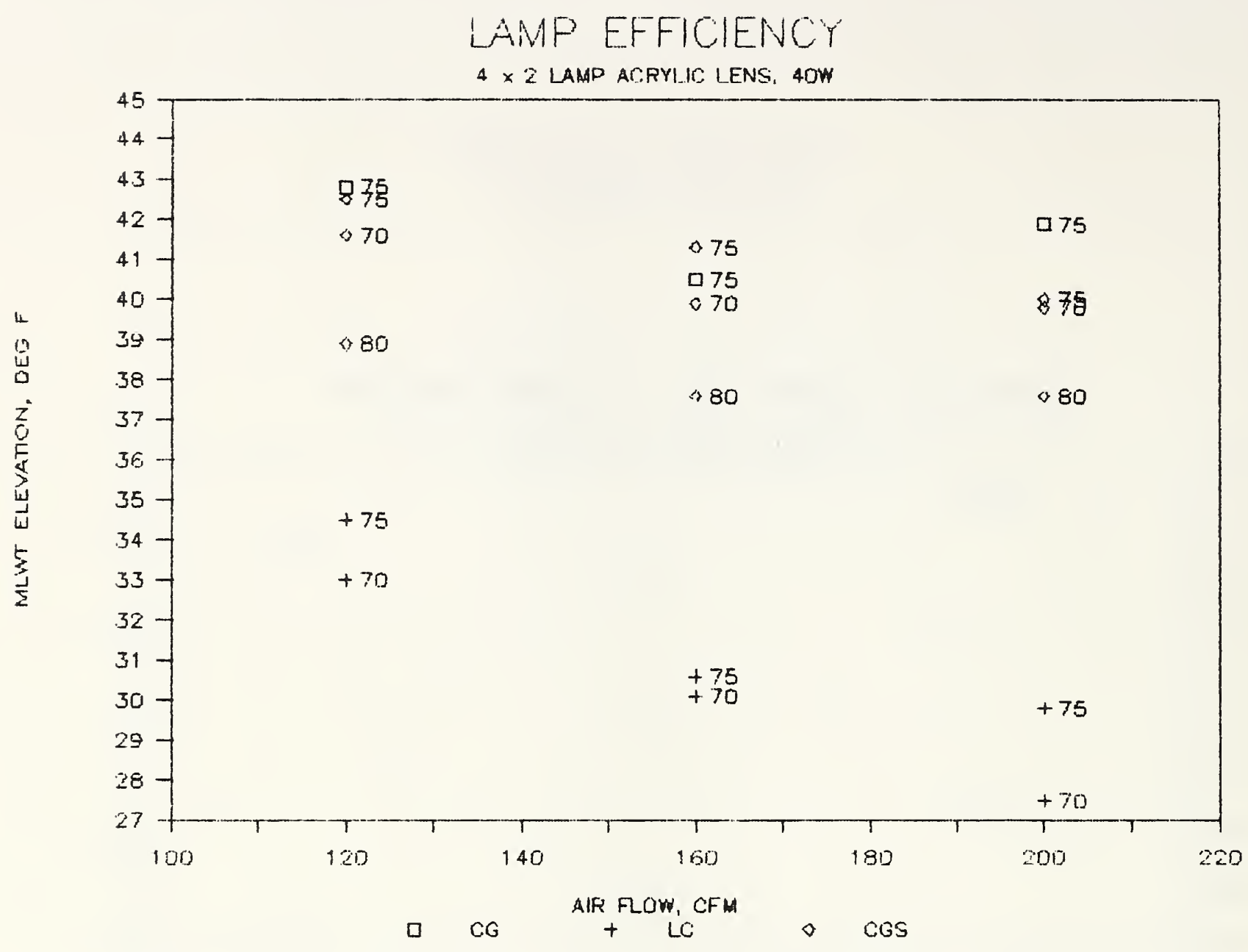

Figure 24. Elevation of minimum lamp wall temperature above room air temperature for four two-lamp acrylic lens luminaires 


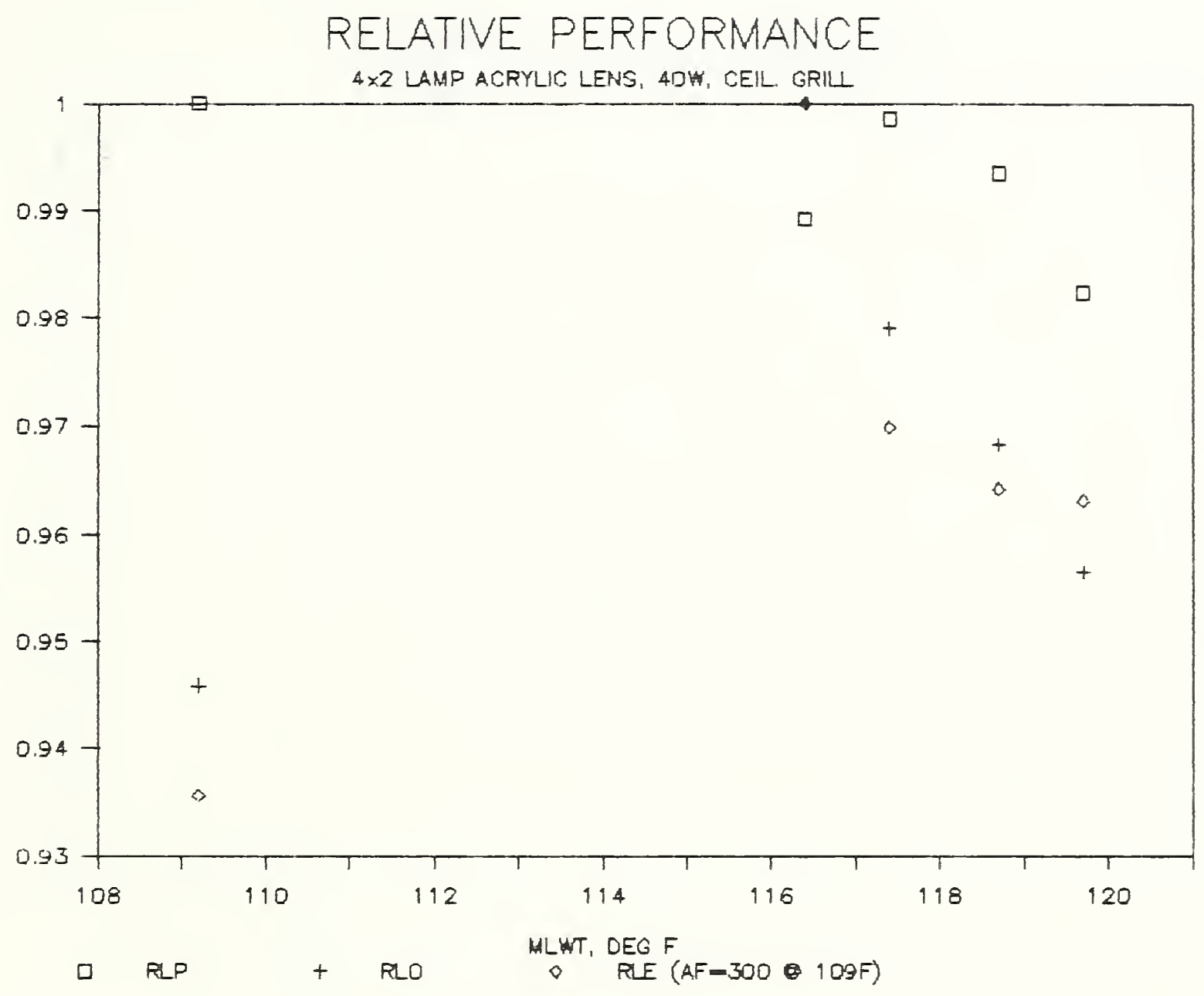

Figure 25. Lighting power, light output, and luminous efficacy for four two-lamp acrylic lens luminaires with ceiling grill return 


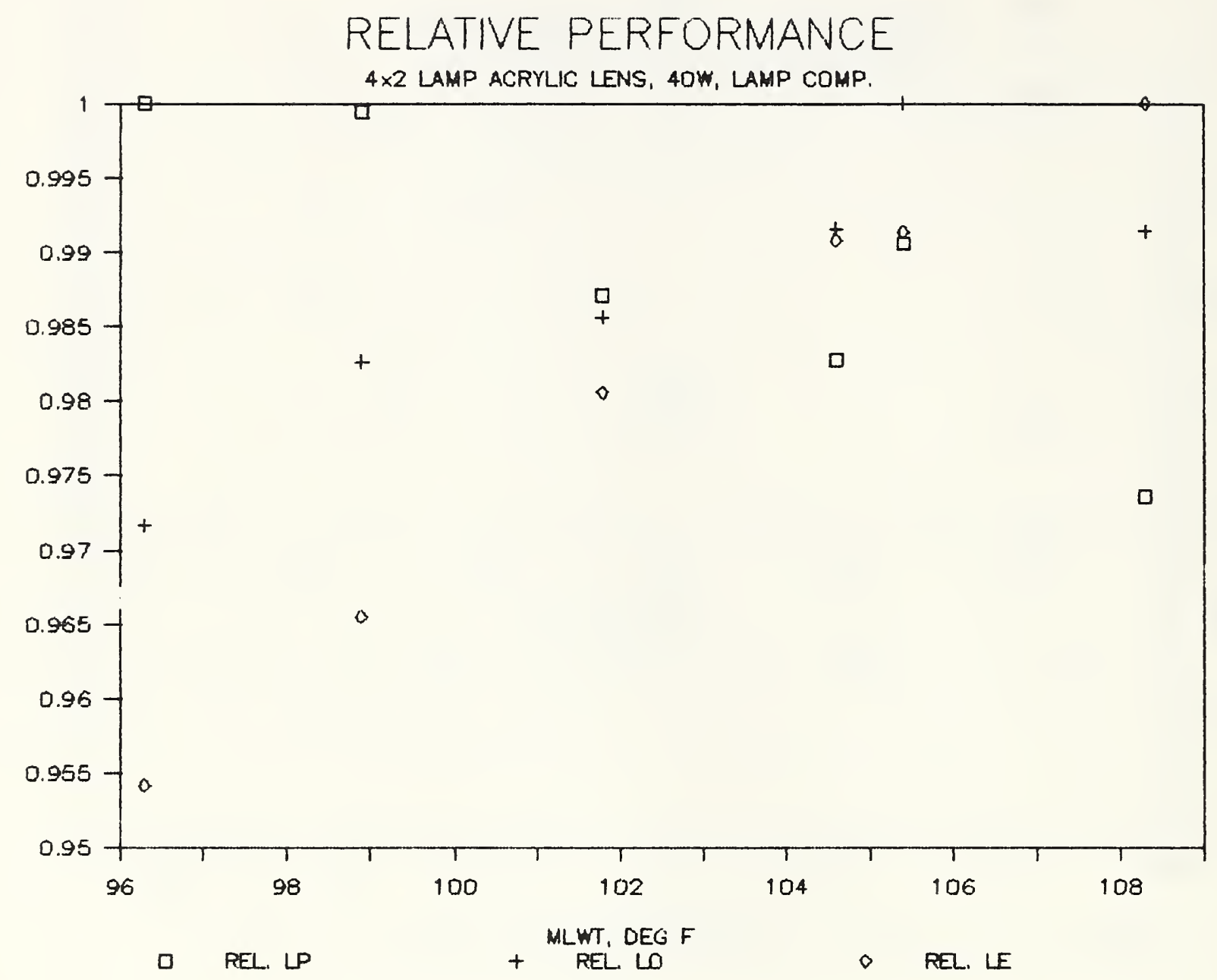

Figure 26. Lighting power, light output, and luminous efficacy for four two-lamp acrylic lens luminaires with lamp compartment return 


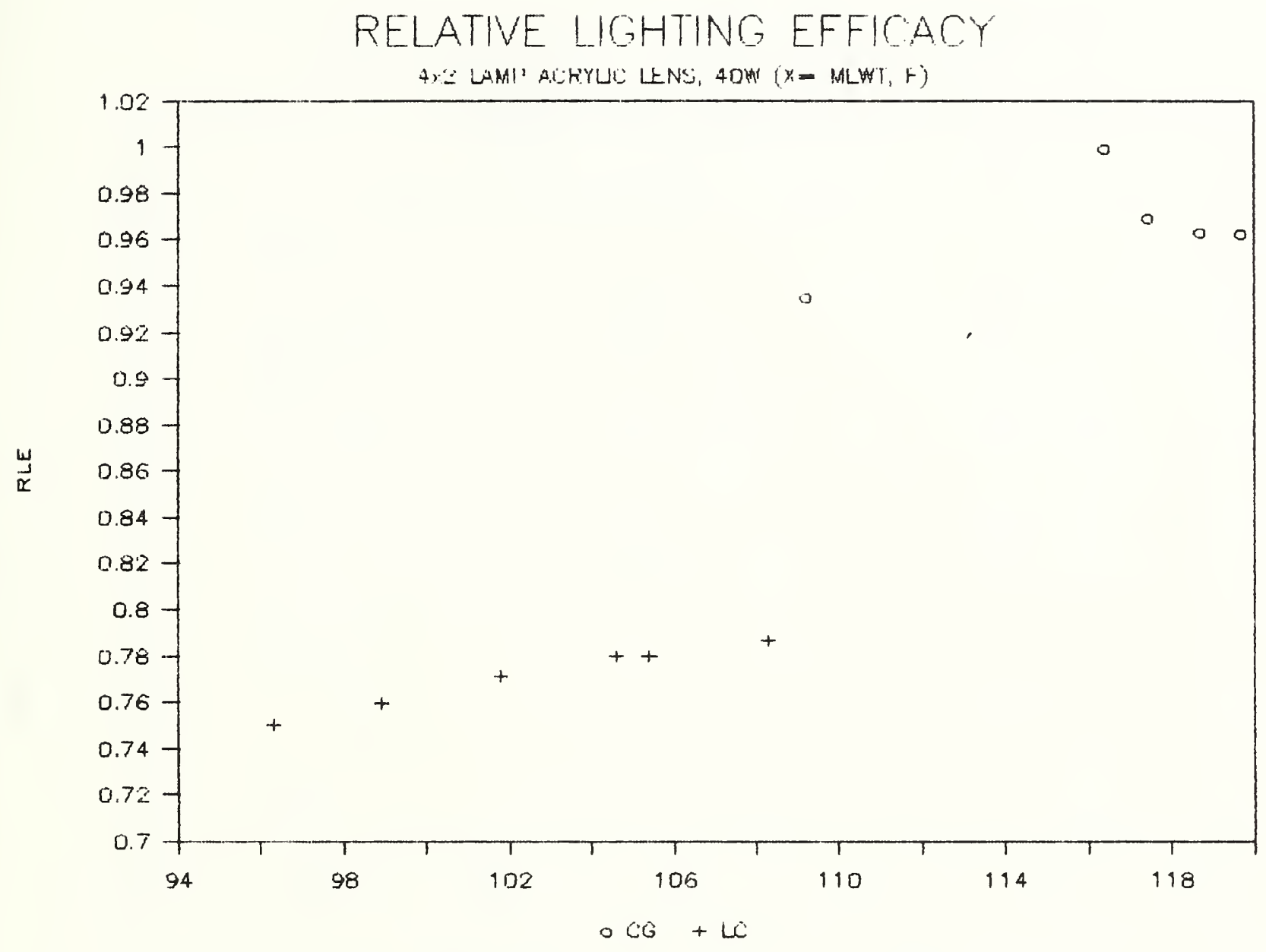

Figure 27. Luminous efficacy for four two-lamp acrylic lens luminaires with ceiling grill and lamp compartment return 


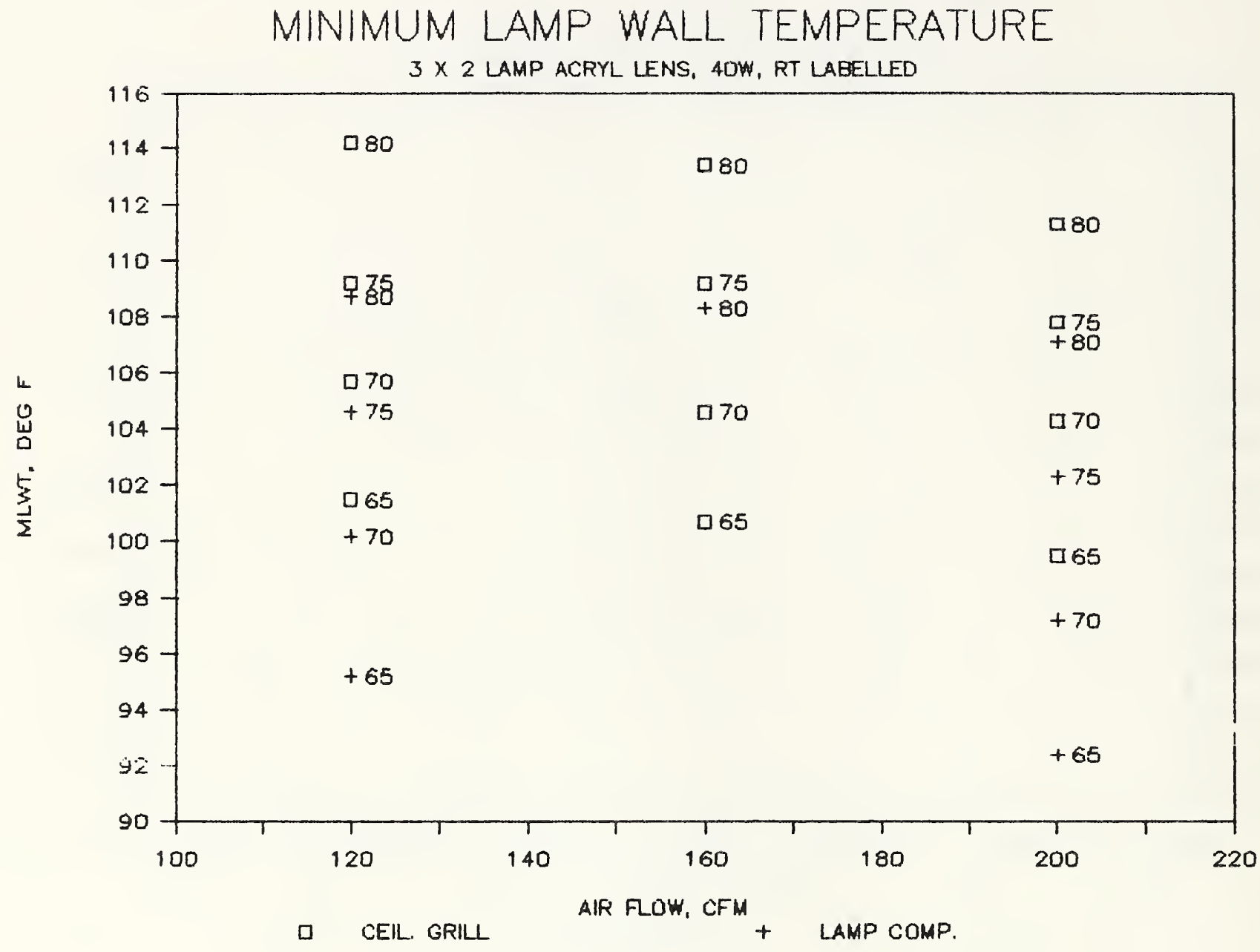

Figure 28. Minimum lamp wall temperature for three two-lamp acrylic lens luminaires with ceiling grill or lamp compartment return 


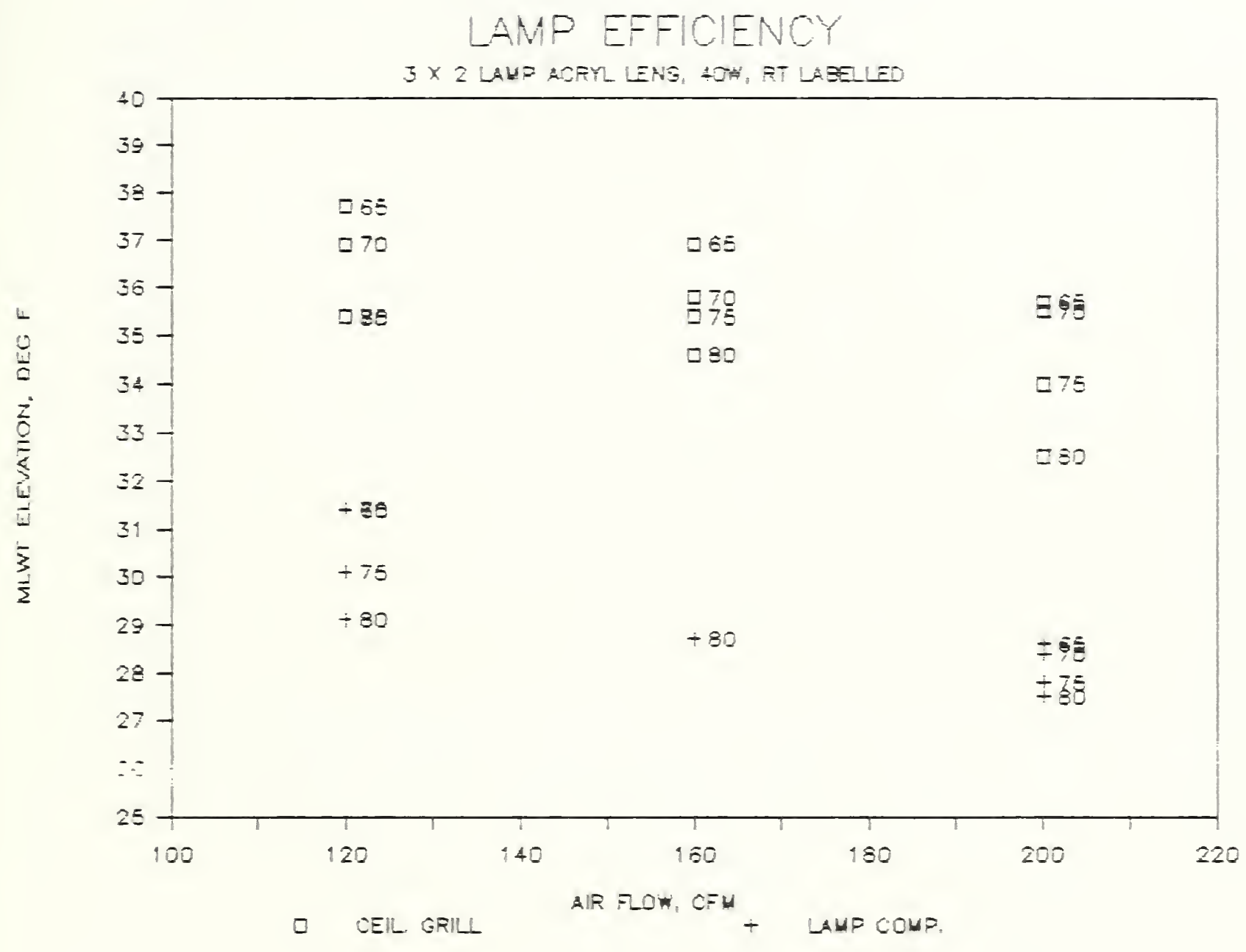

Figure 29. Eievation of minimum lamp wall temperature above room air temperature for three two-lamp acrylic lens luminaires 
RELATIVE PERFORMANCE

$3 \times 2$ LAMP ACRYL LENS. 4DW, CEII. GRILL

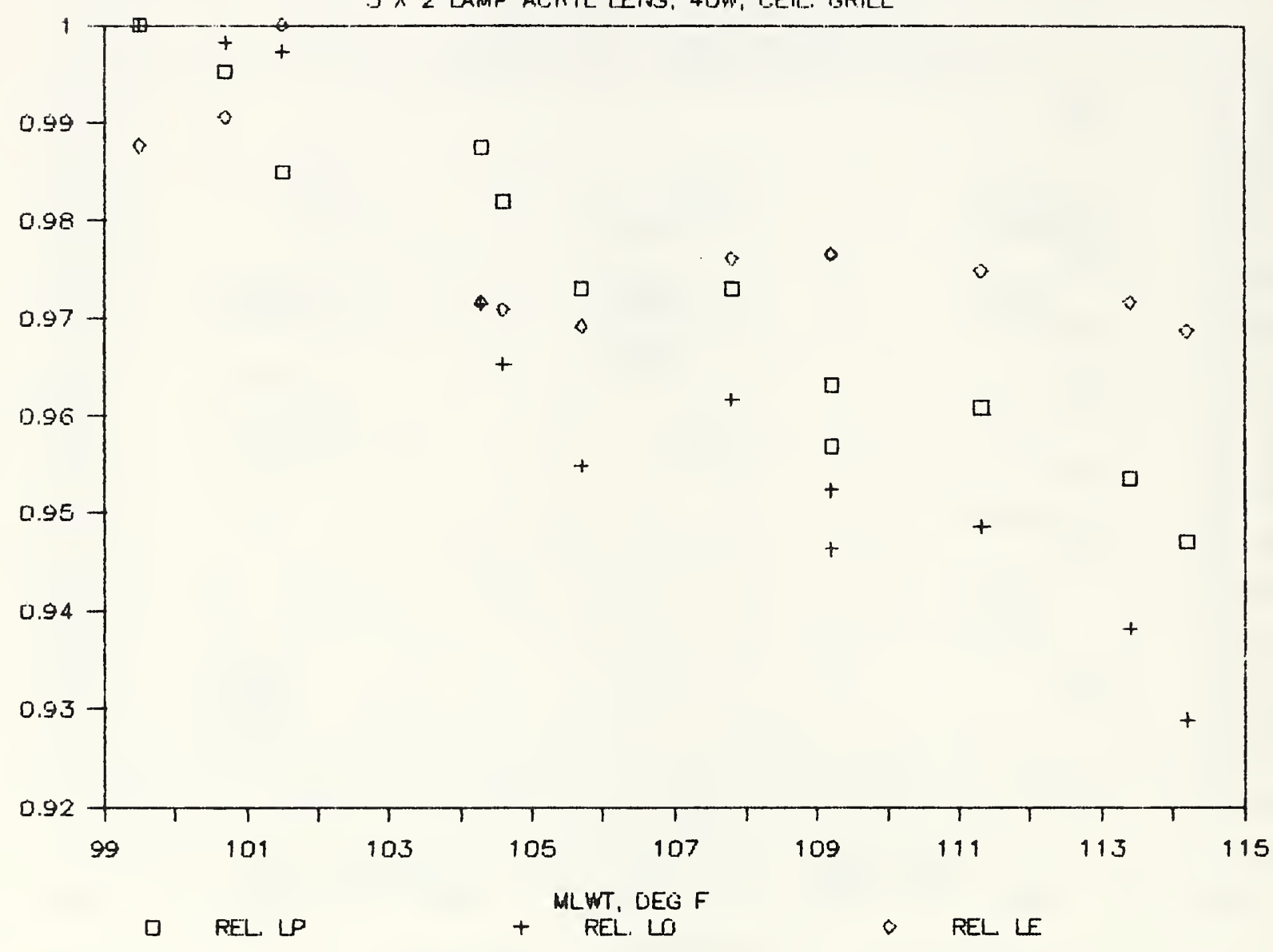

Figure 30. Lighting power, light output, and luminous efficacy for three two-lamp acrylic lens luminaires with ceiling grill return 


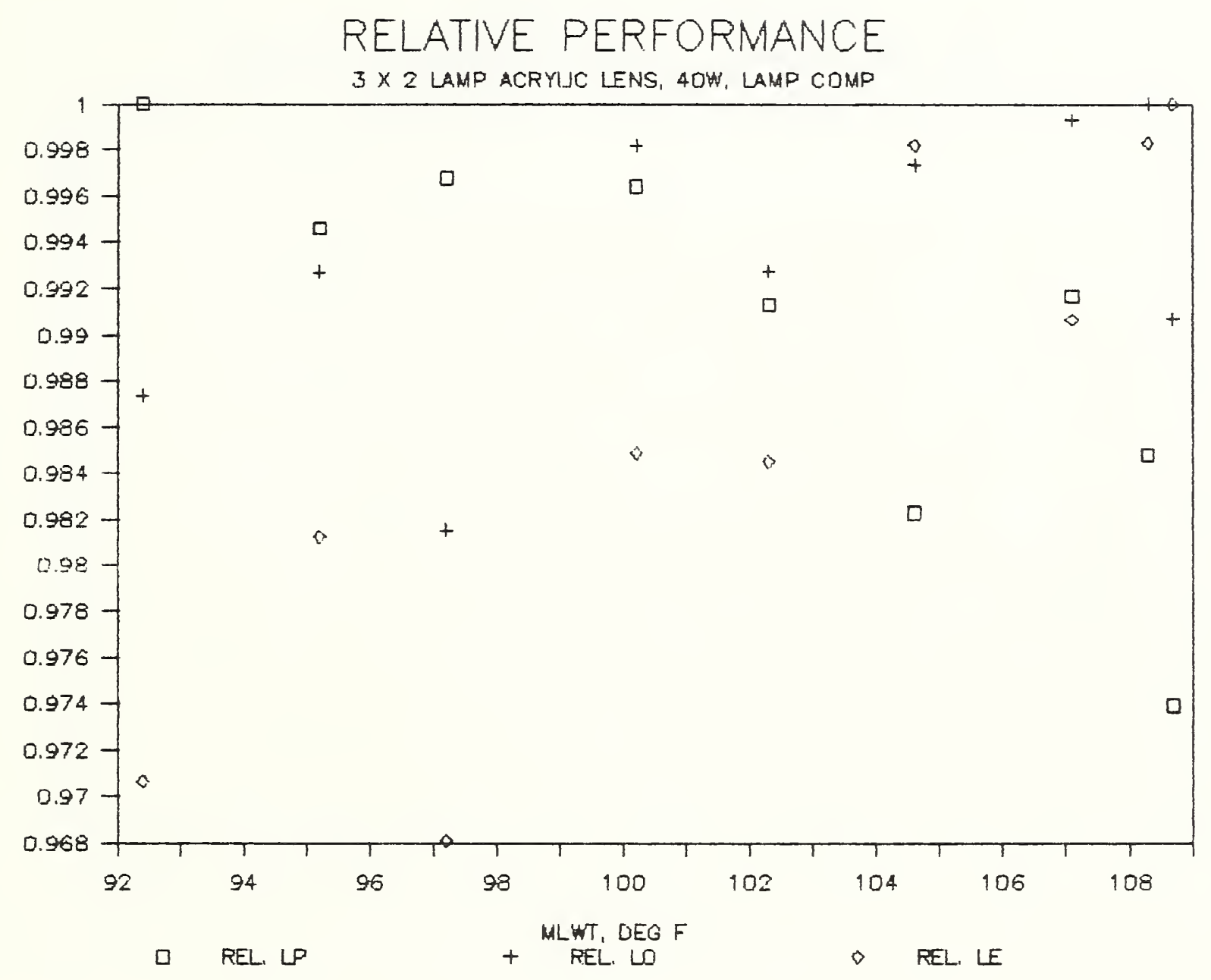

Figure 31. Lighting power, light output, and luminous efficacy for three two-lamp acrylic lens luminaires with lamp compartment return 


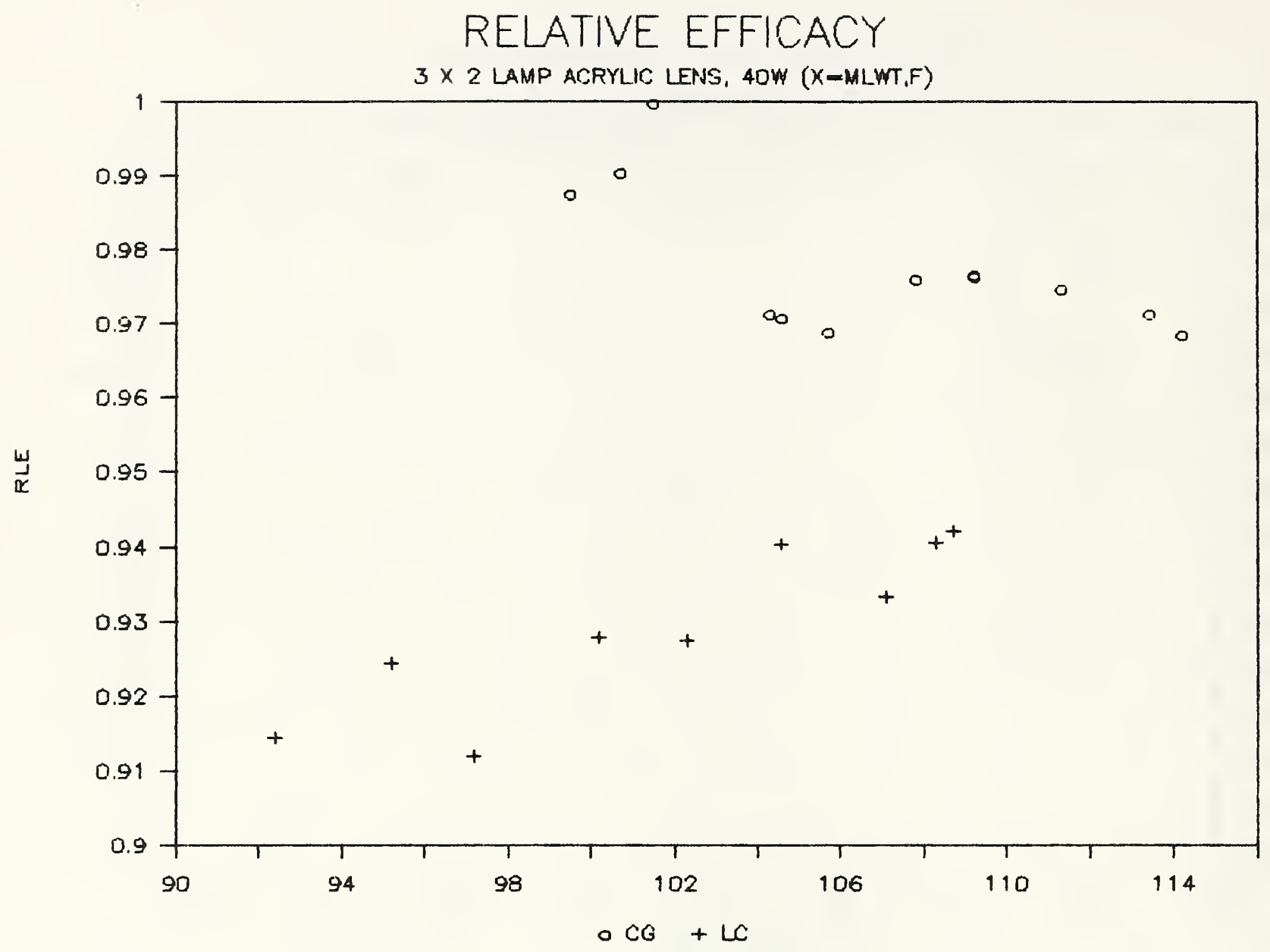

Figure 32. Luminous efficacy for three two-lamp acrylic lens luminaires with ceiling grill and lamp compartment return 


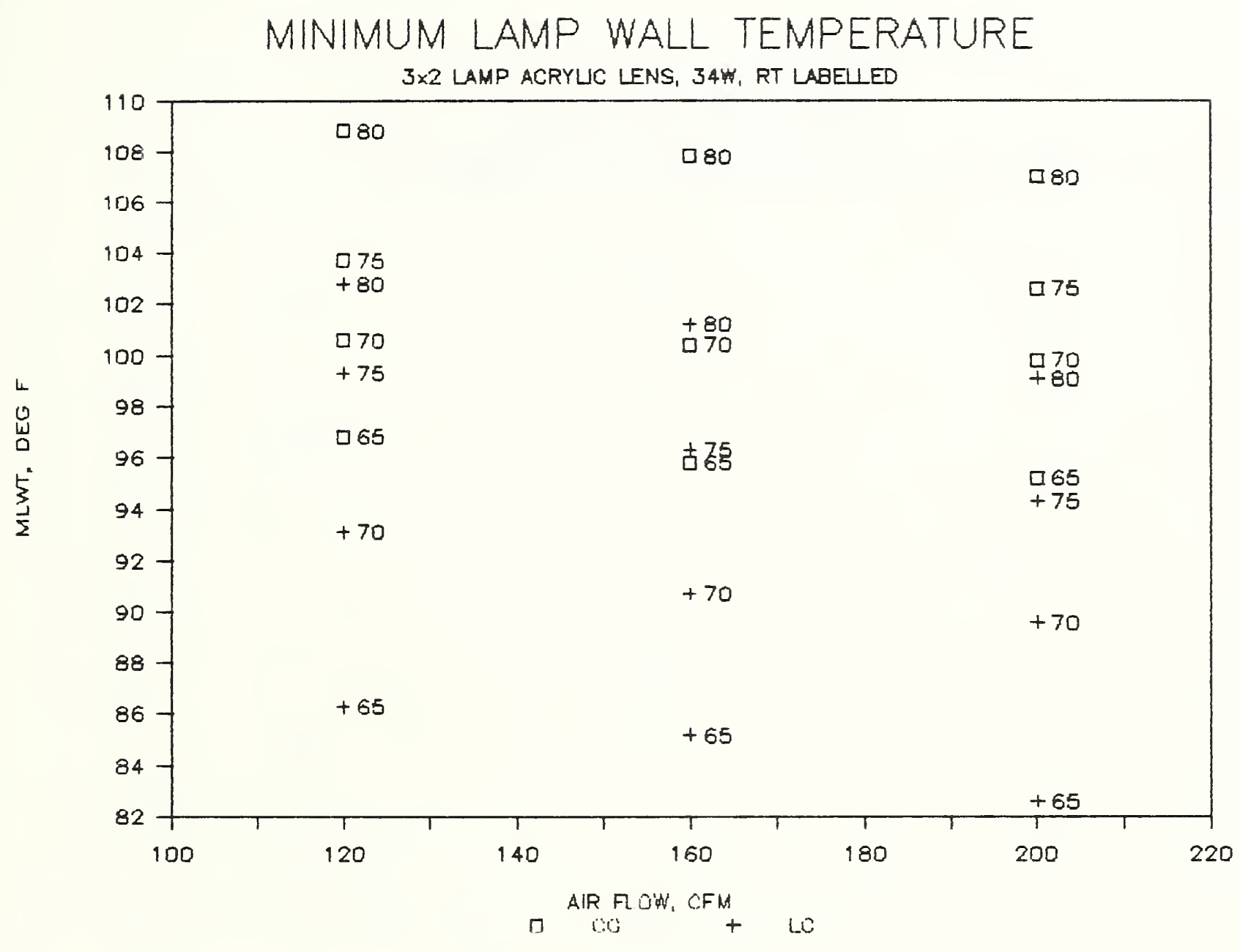

Figure 33. Minimum lamp wall temperature for three two 34-watt lamp acrylic lens luminaires with ceiling grill or lamp compartment return 


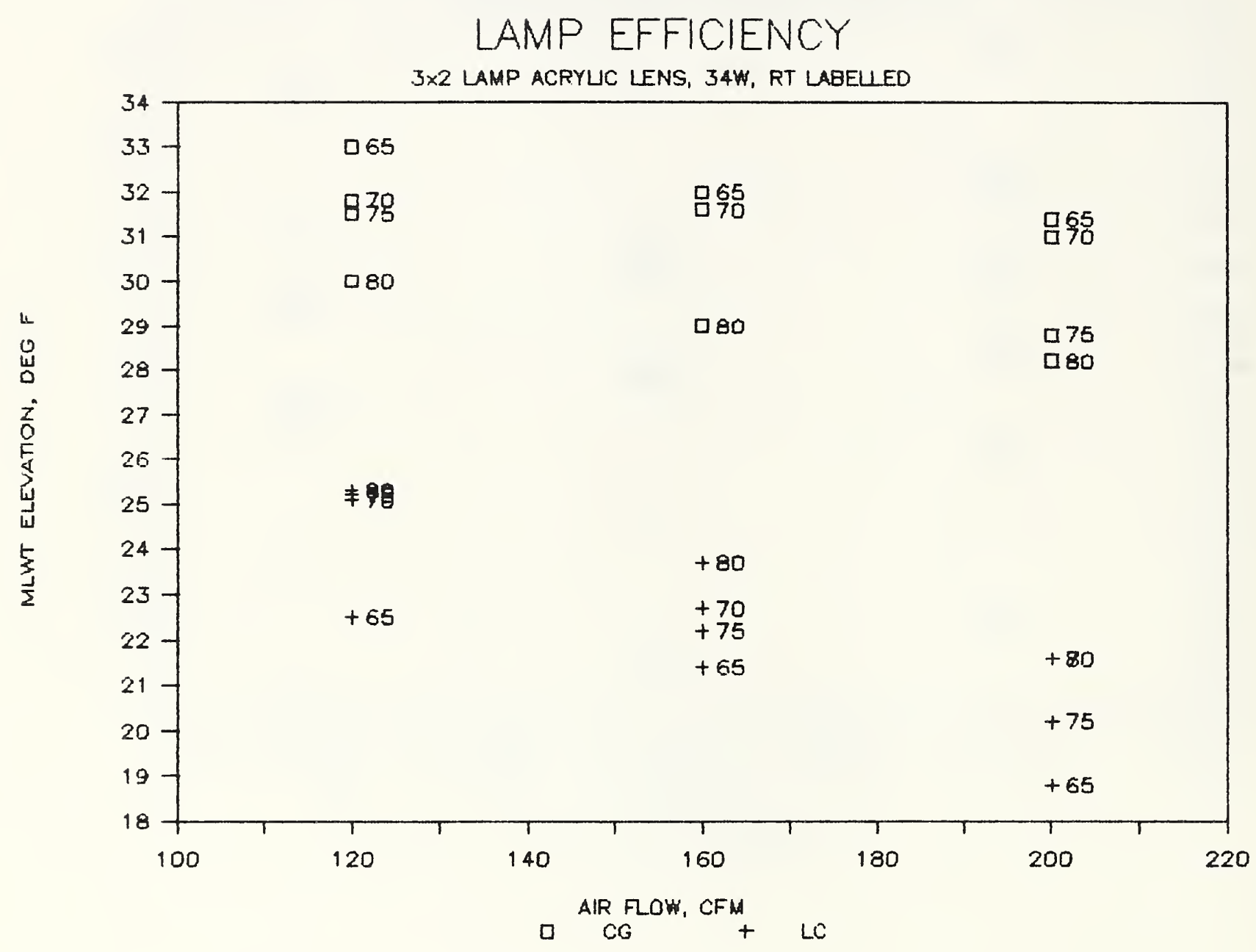

Figure 34. Elevation of minimum lamp wall temperature above room air temperature for three two 34-watt lamp acrylic lens luminaires 


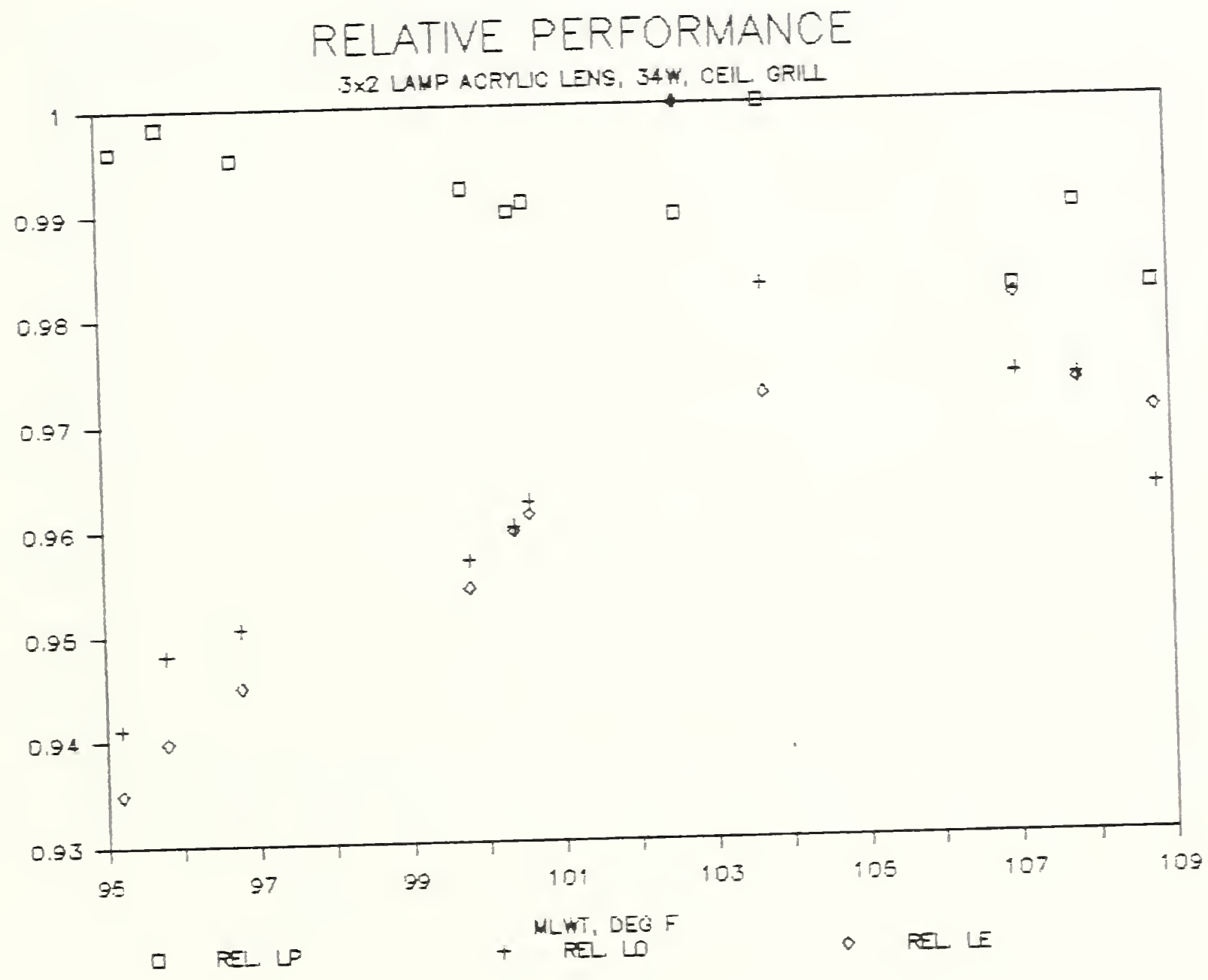

Figure 35. Lighting power, light output, and luminous efficacy for three two 34-watt lamp acrylic lens luminaires with ceiling grill return 
RELATIVE PERFORMANCE

$3 \times 2$ LAMP ACRYLIC LENS, 34W, LAMP COMP.

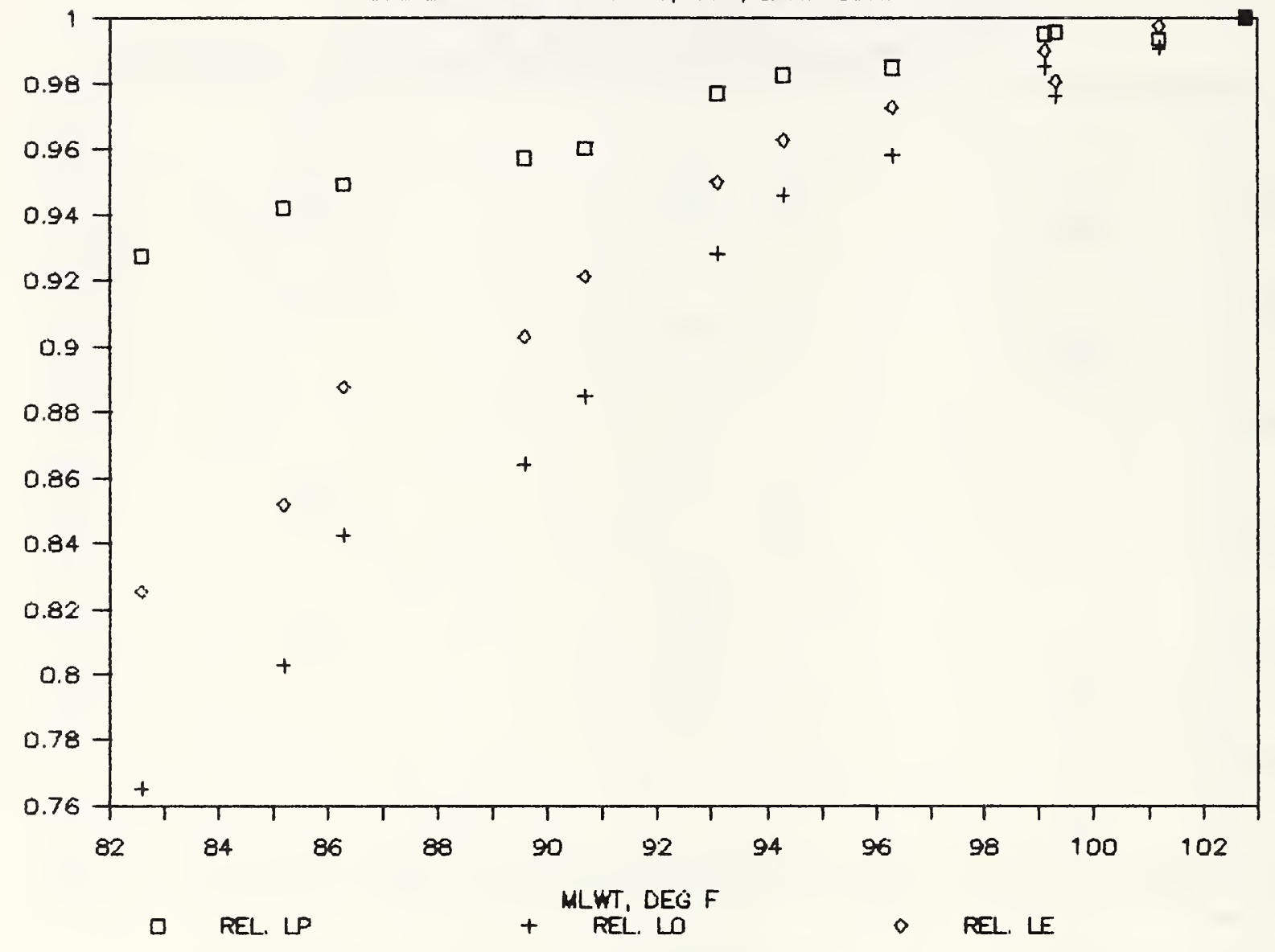

Figure 36. Lighting power, light output, and luminous efficacy for three two 34-watt lamp acrylic lens luminaires with lamp compartment return 


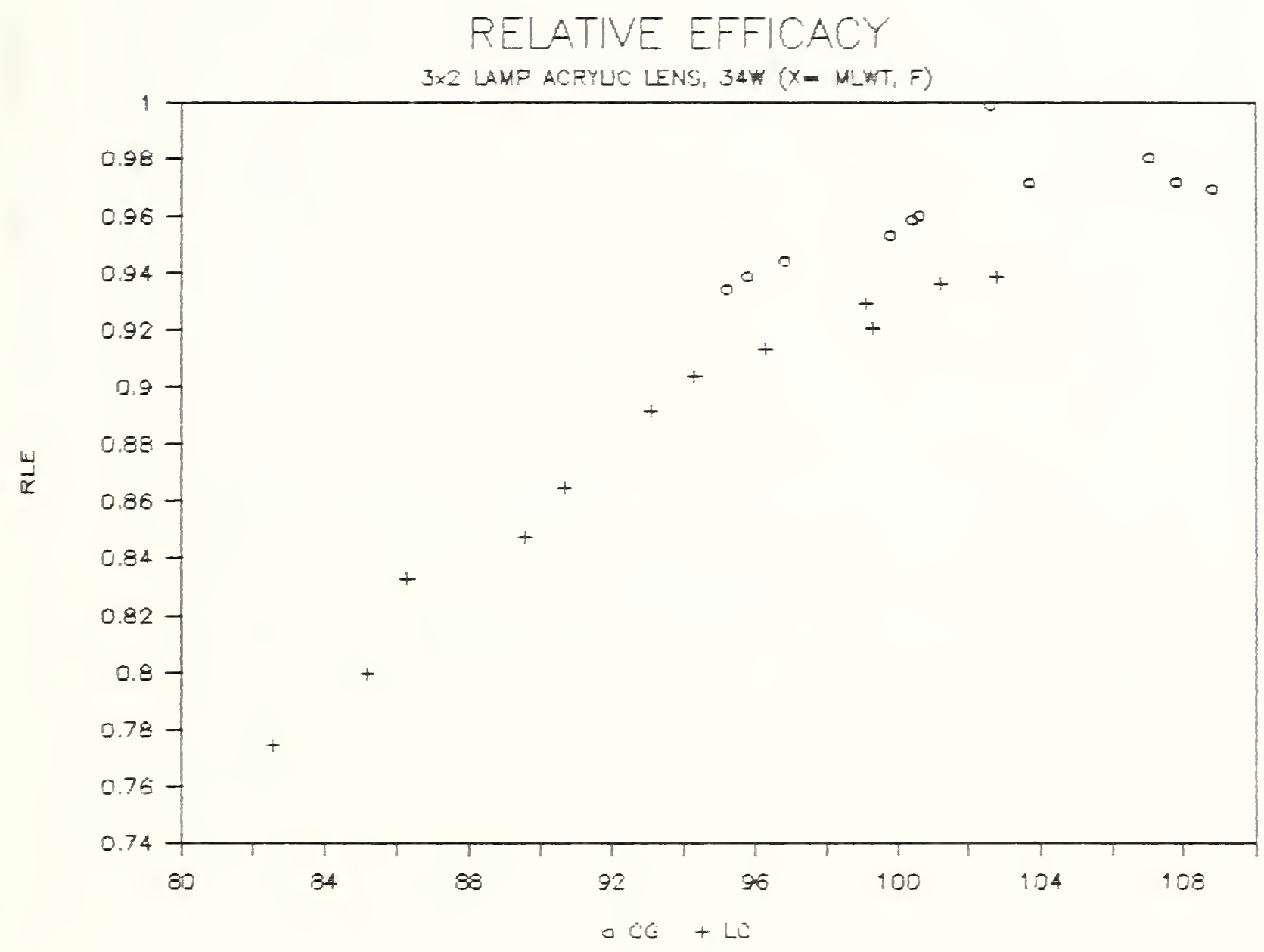

Figure 37. Luminous efficacy for three two 34-watt lamp acrylic lens luminaires with ceiling grill and lamp compartment retum 
EFFECT OF AIRFLOW, RETURN PATH

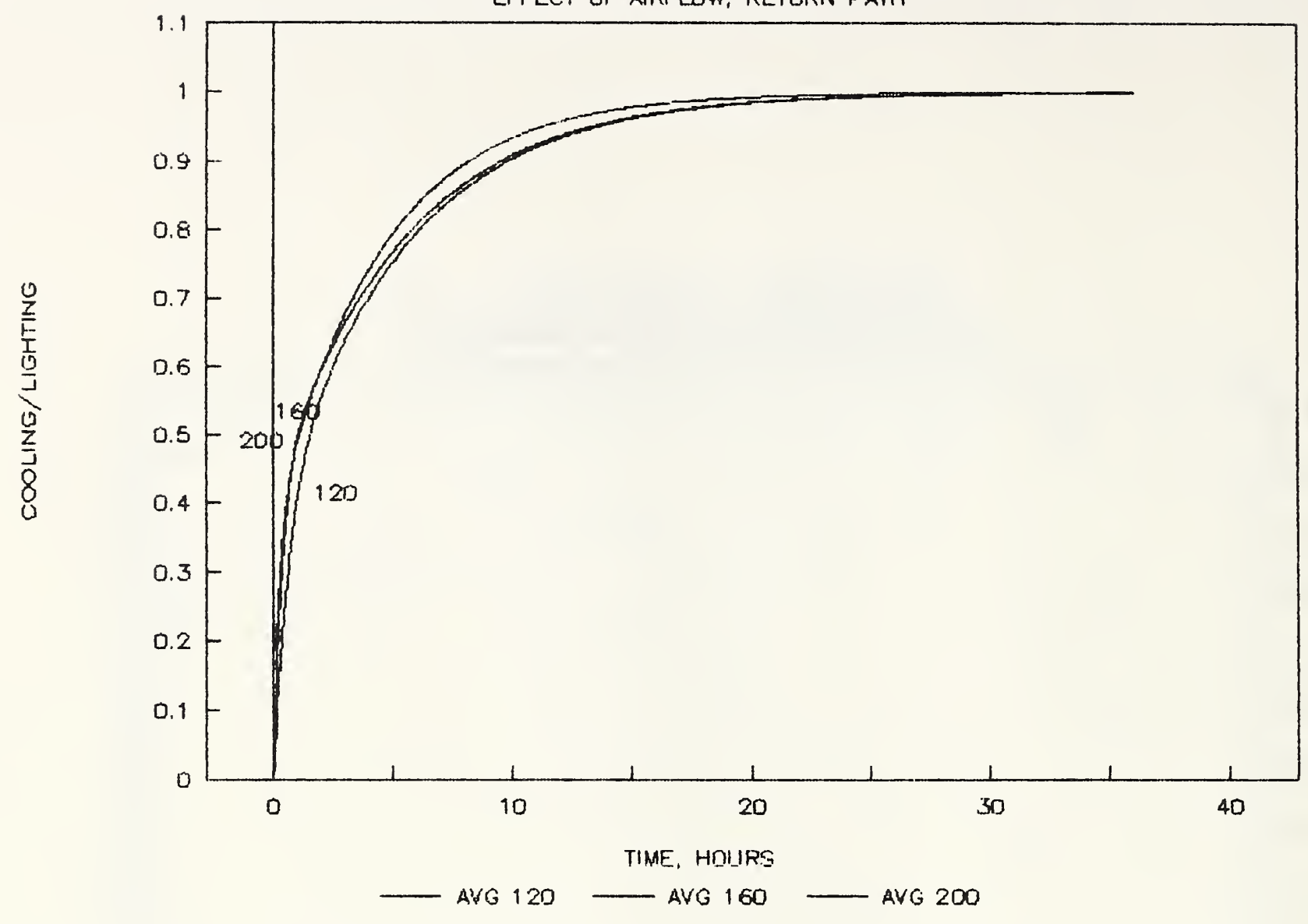

Figure 38. Cooling load regression for the four lamp parabolic diffuser luminaires with lamp compartment return 


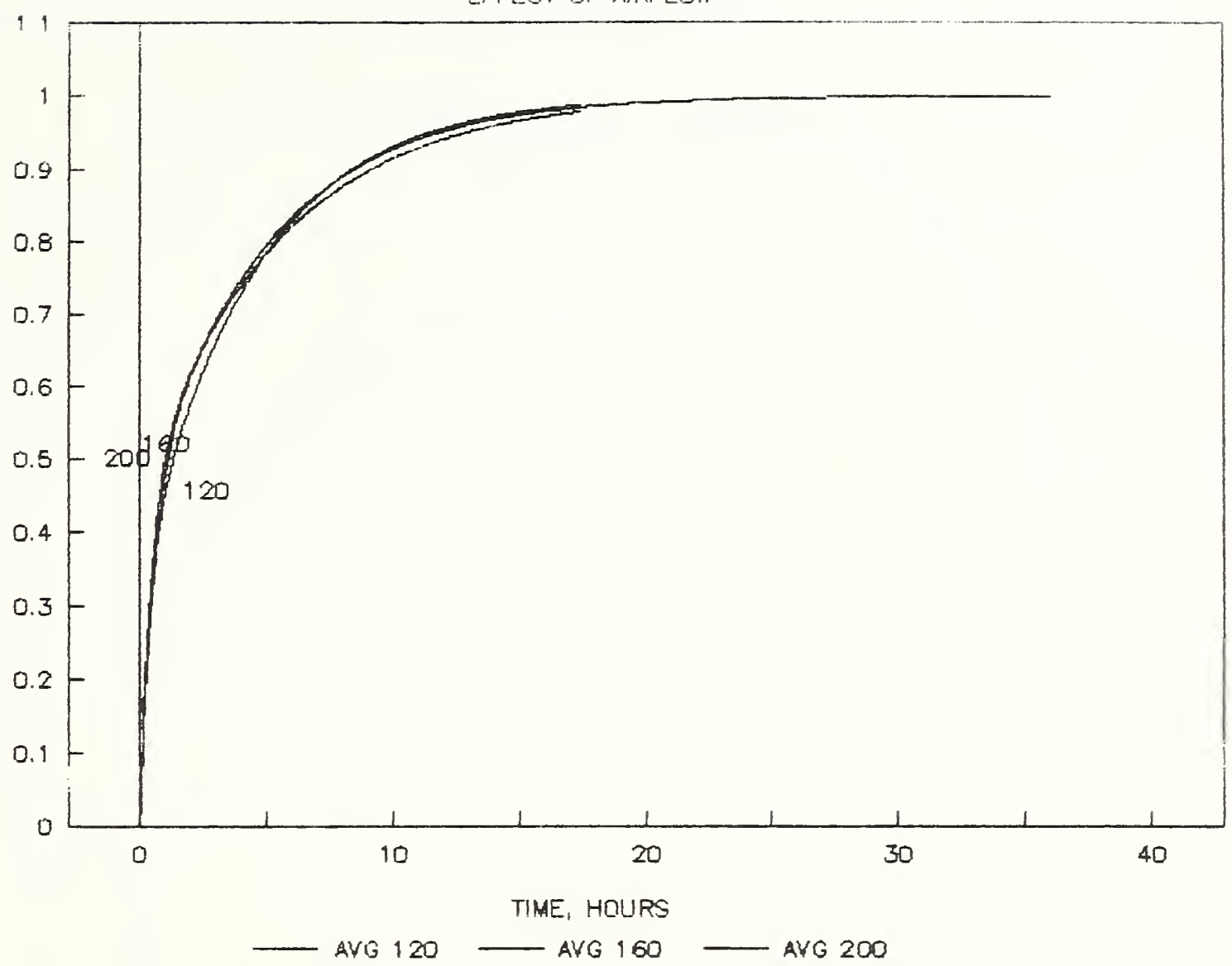

Figure 39. Cooling load regression for the four lamp parabolic diffuser luminaires with ceiling grill return 


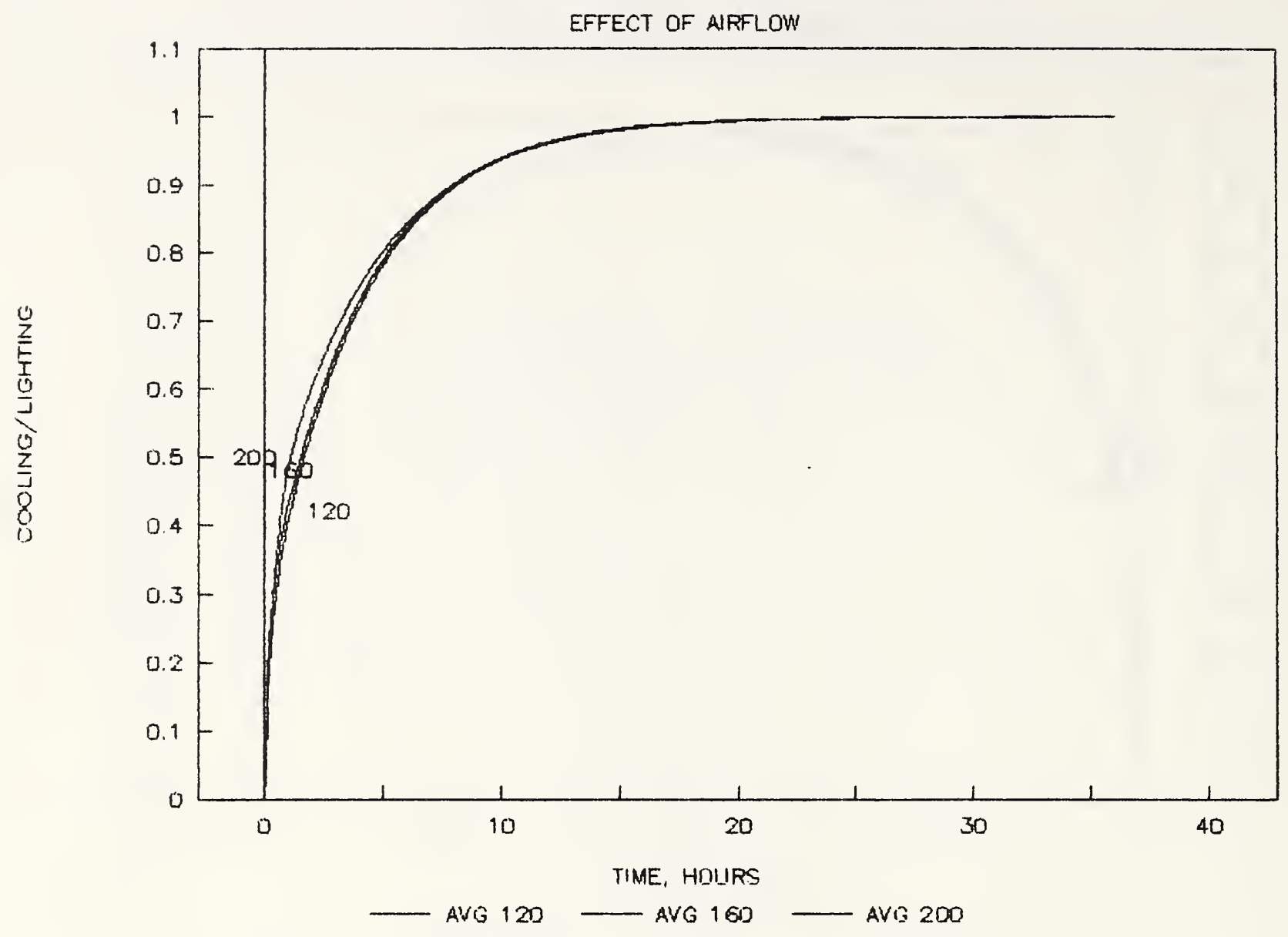

Figure 40. Cooling load regression for the four lamp parabolic diffuser luminaires with side slot return 


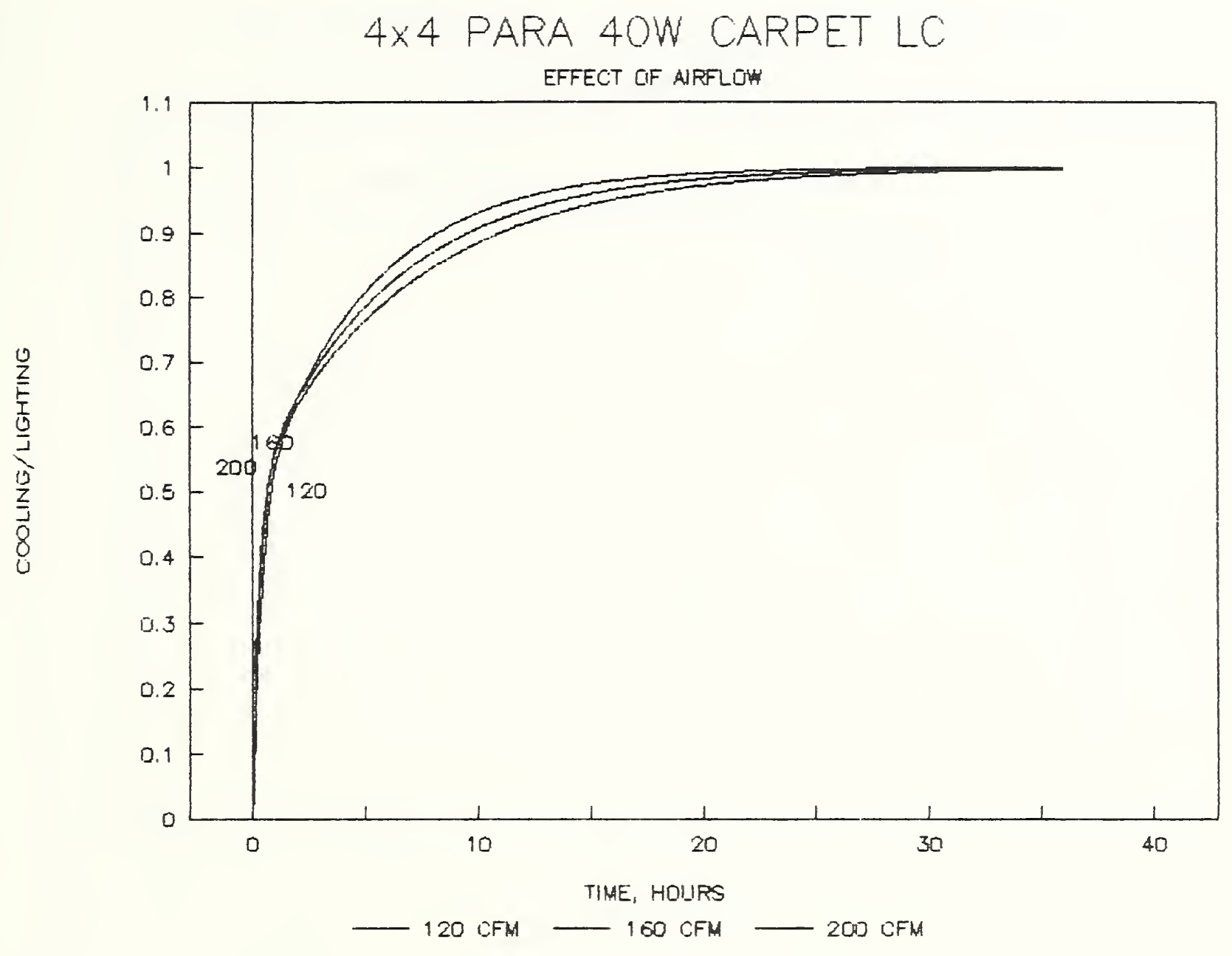

Figure 41. Cooling load regression for the four lamp parabolic diffuser luminaires with lamp compartment return, carpet 


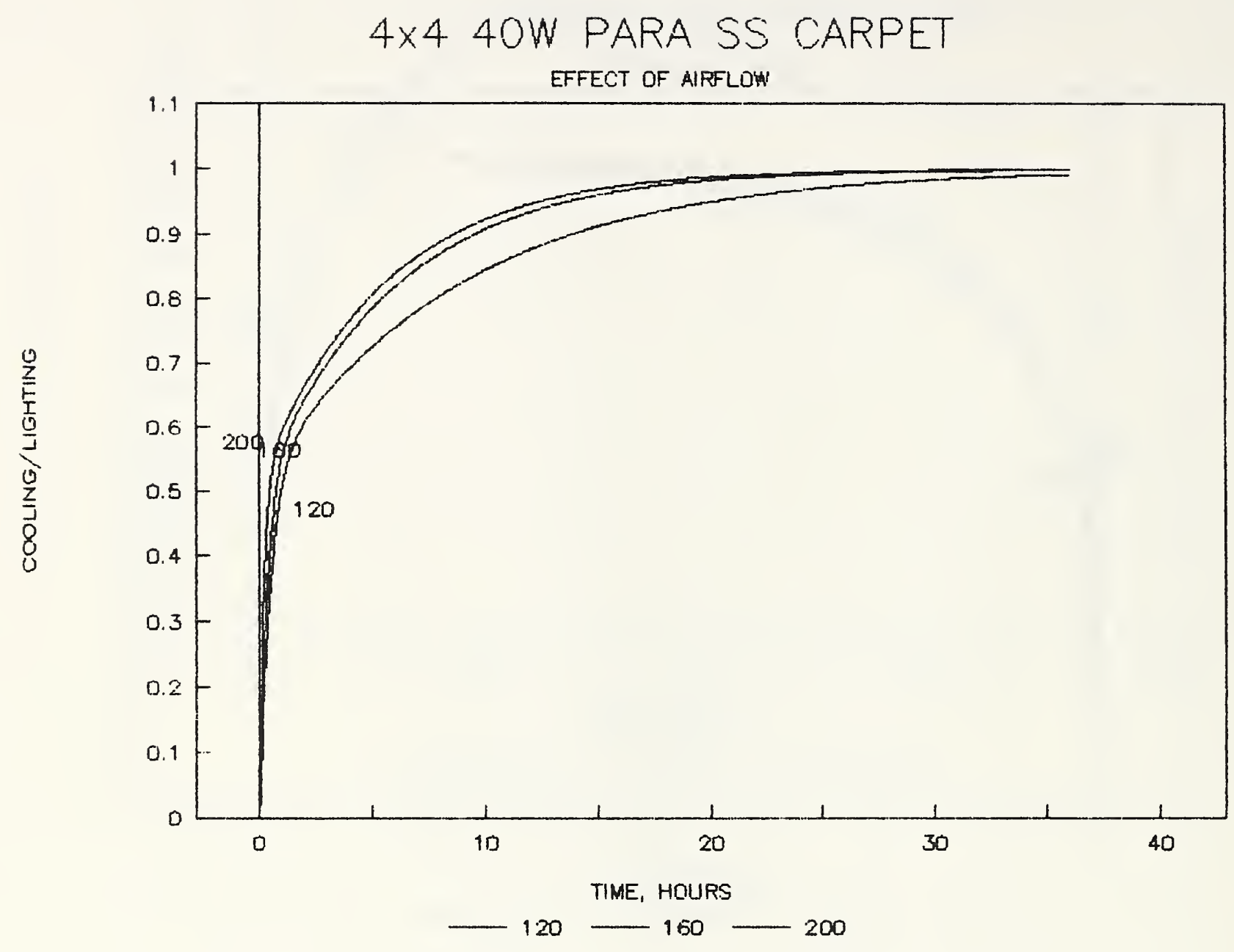

Figure 42. Cooling load regression for the four lamp parabolic diffuser luminaires with side slot return, carpet 


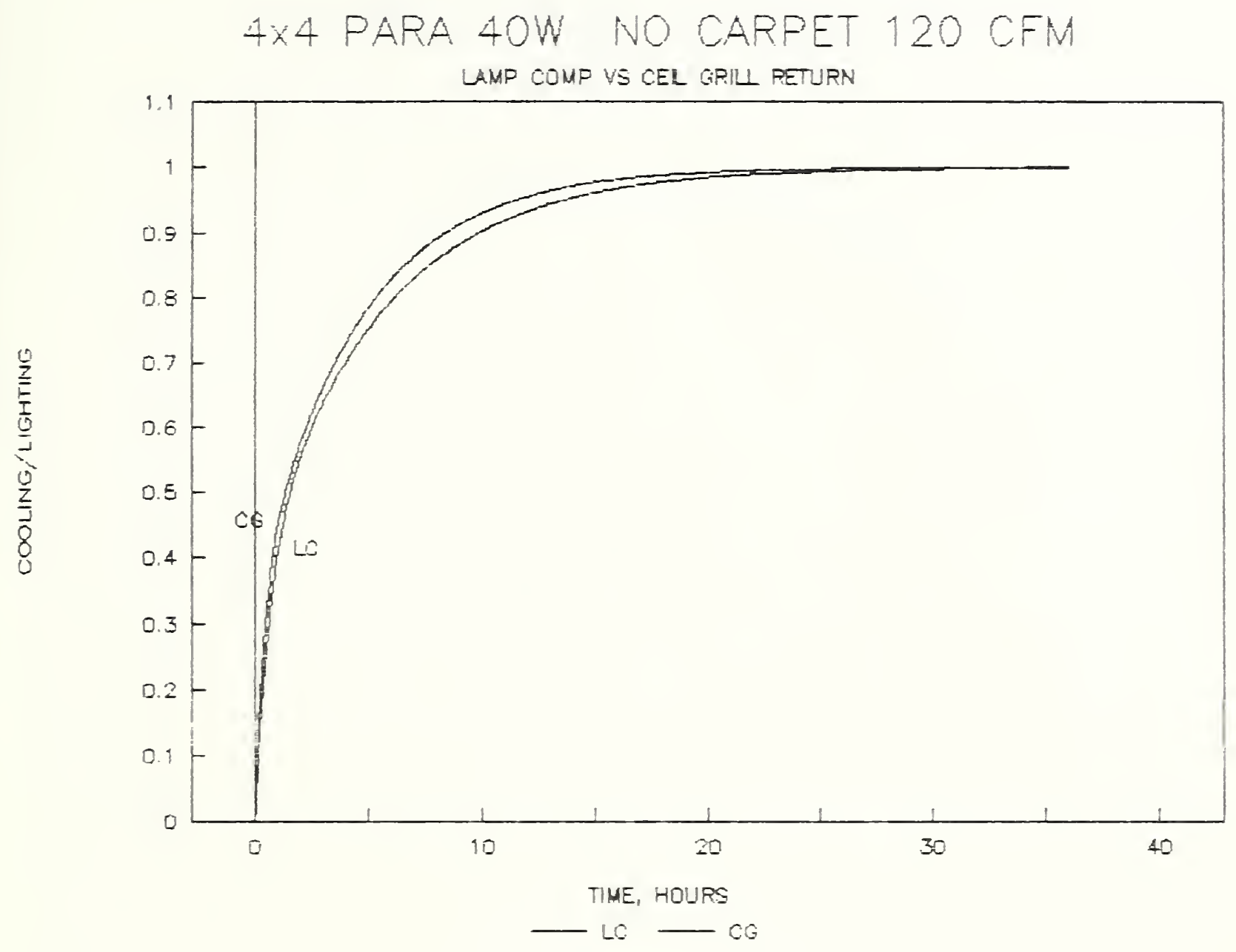

Figure 43. Cooling load regression for the four lamp parabolic diffuser luminaires, $120 \mathrm{cfm}$ airflow, lamp compartment and ceiling grill return 


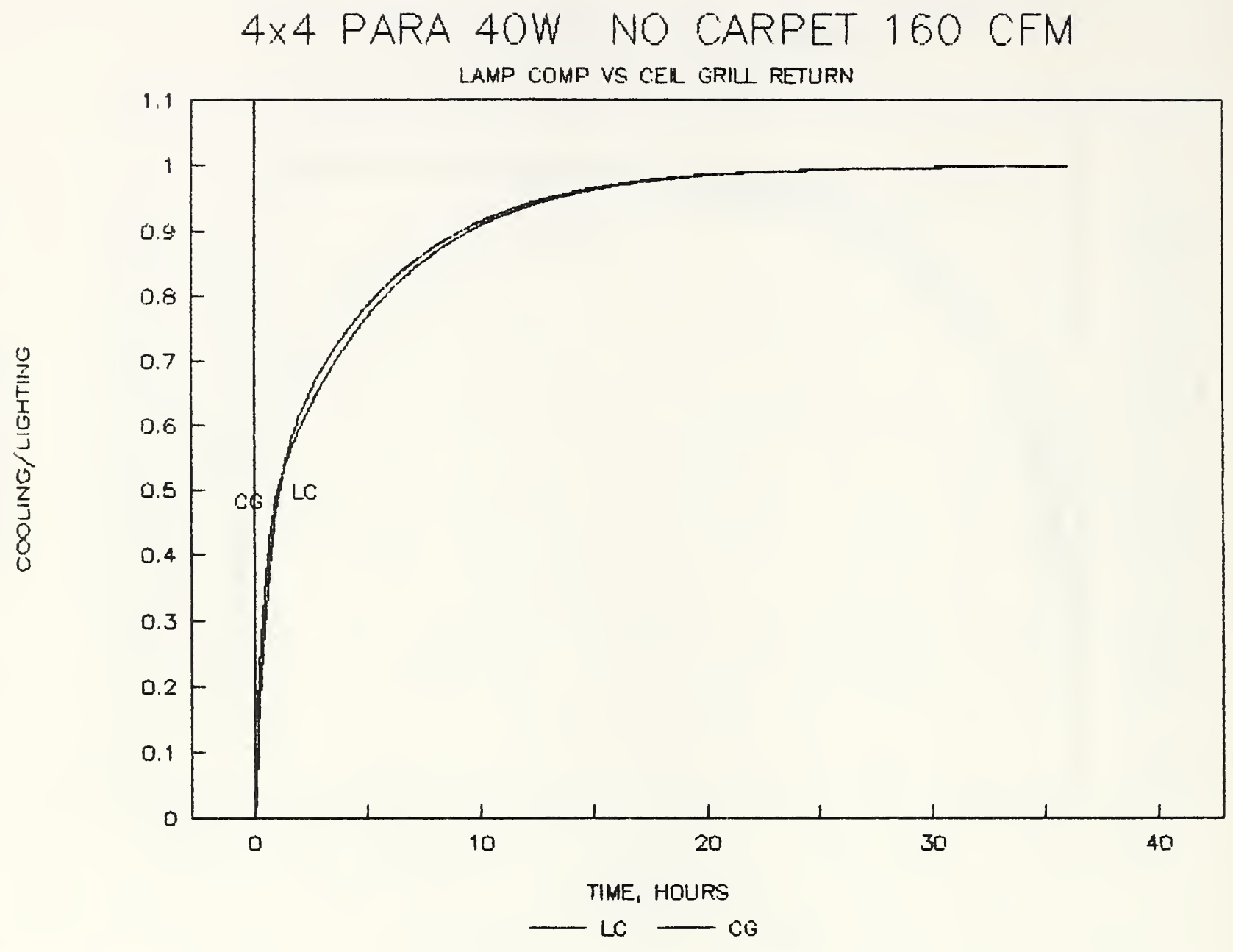

Figure 44. Cooling load regression for the four lamp parabolic diffuser luminaires, $160 \mathrm{cfm}$ airflow, lamp compartment and ceiling grill return 


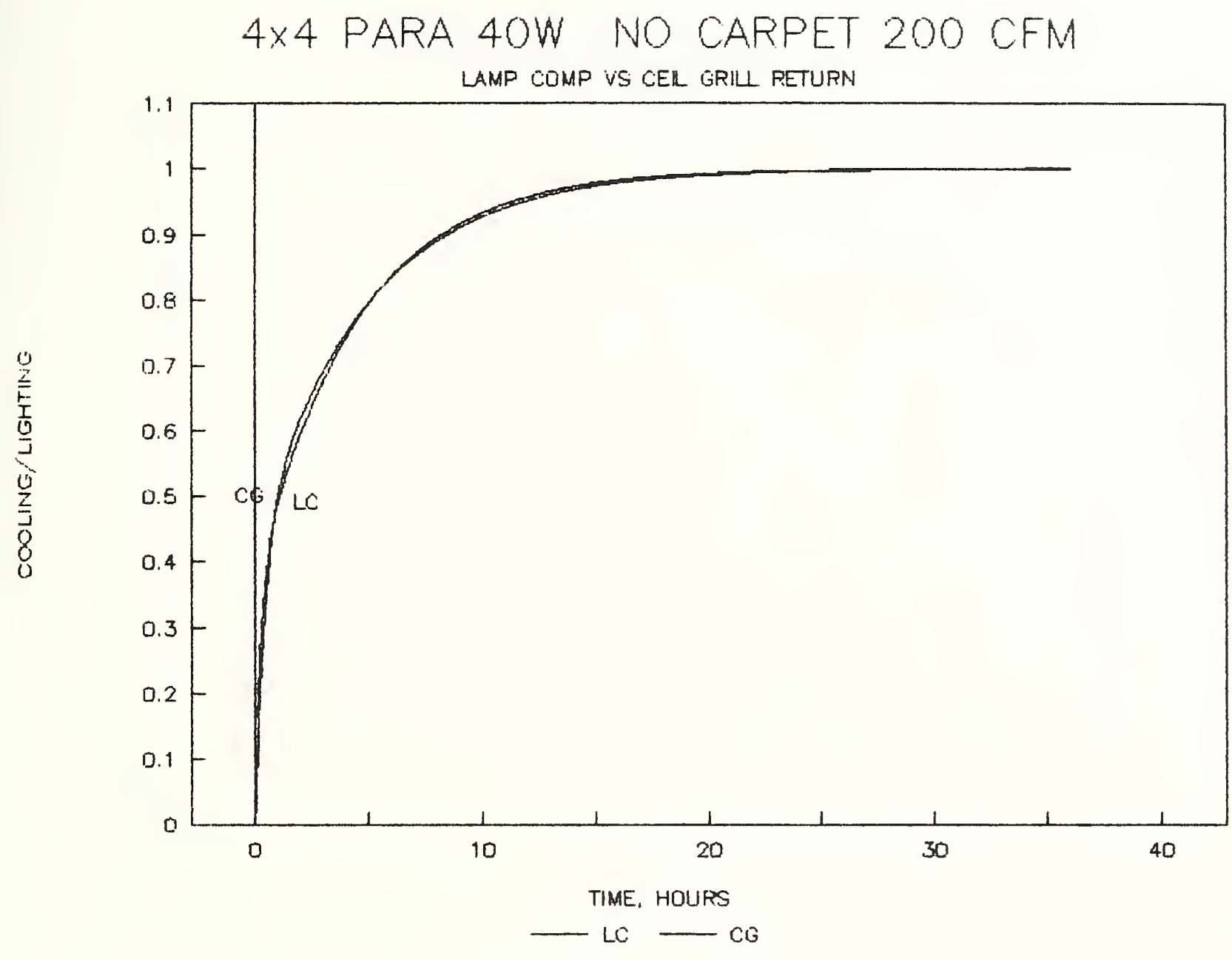

Figure 45. Cooling load regression for the four lamp parabolic diffuser luminaires, $200 \mathrm{cfm}$ airflow, lamp compartment and ceiling grill return 


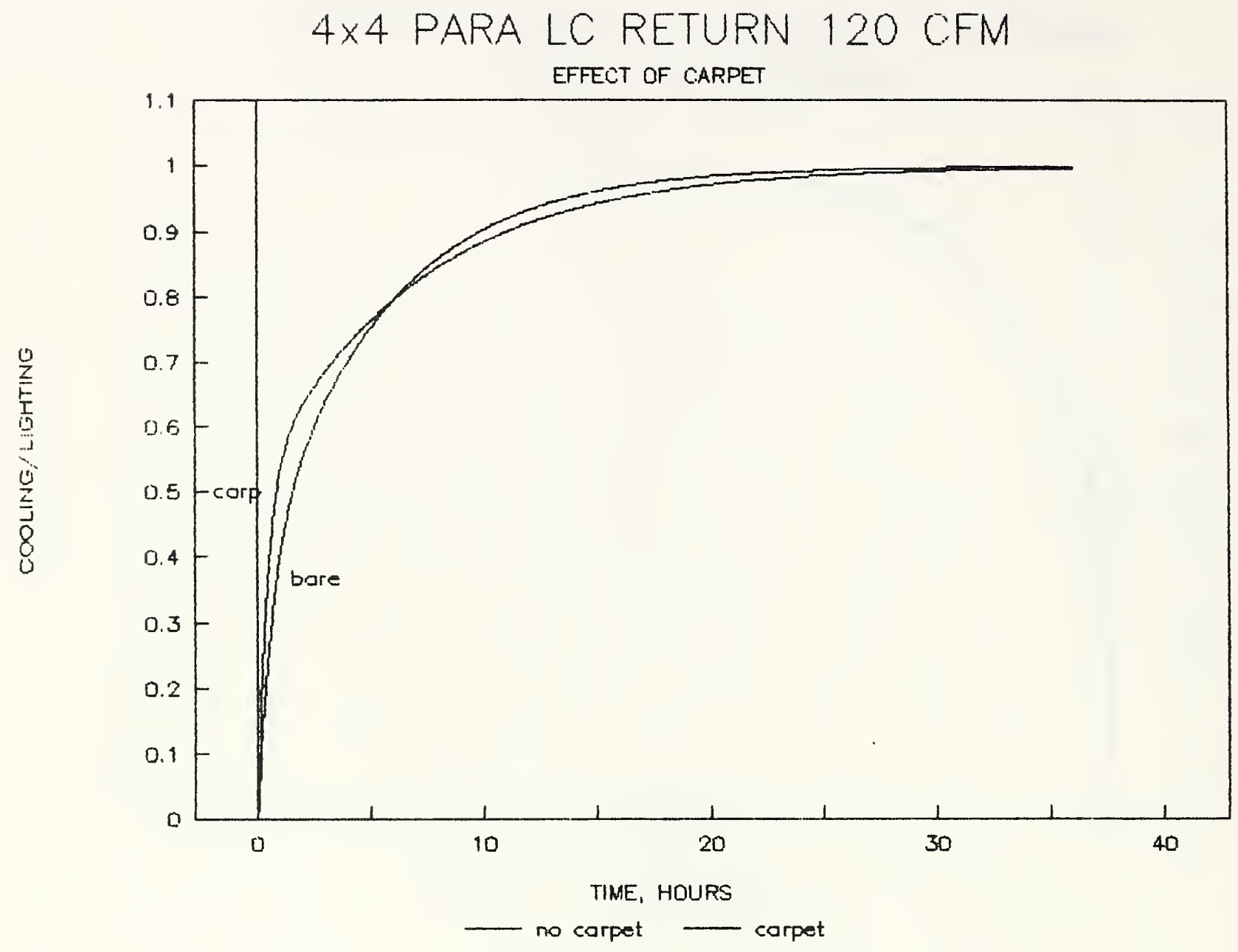

Figure 46. Cooling load regression for the four lamp parabolic diffuser luminaires, $120 \mathrm{cfm}$ airflow, lamp compartment return, with and without carpet 


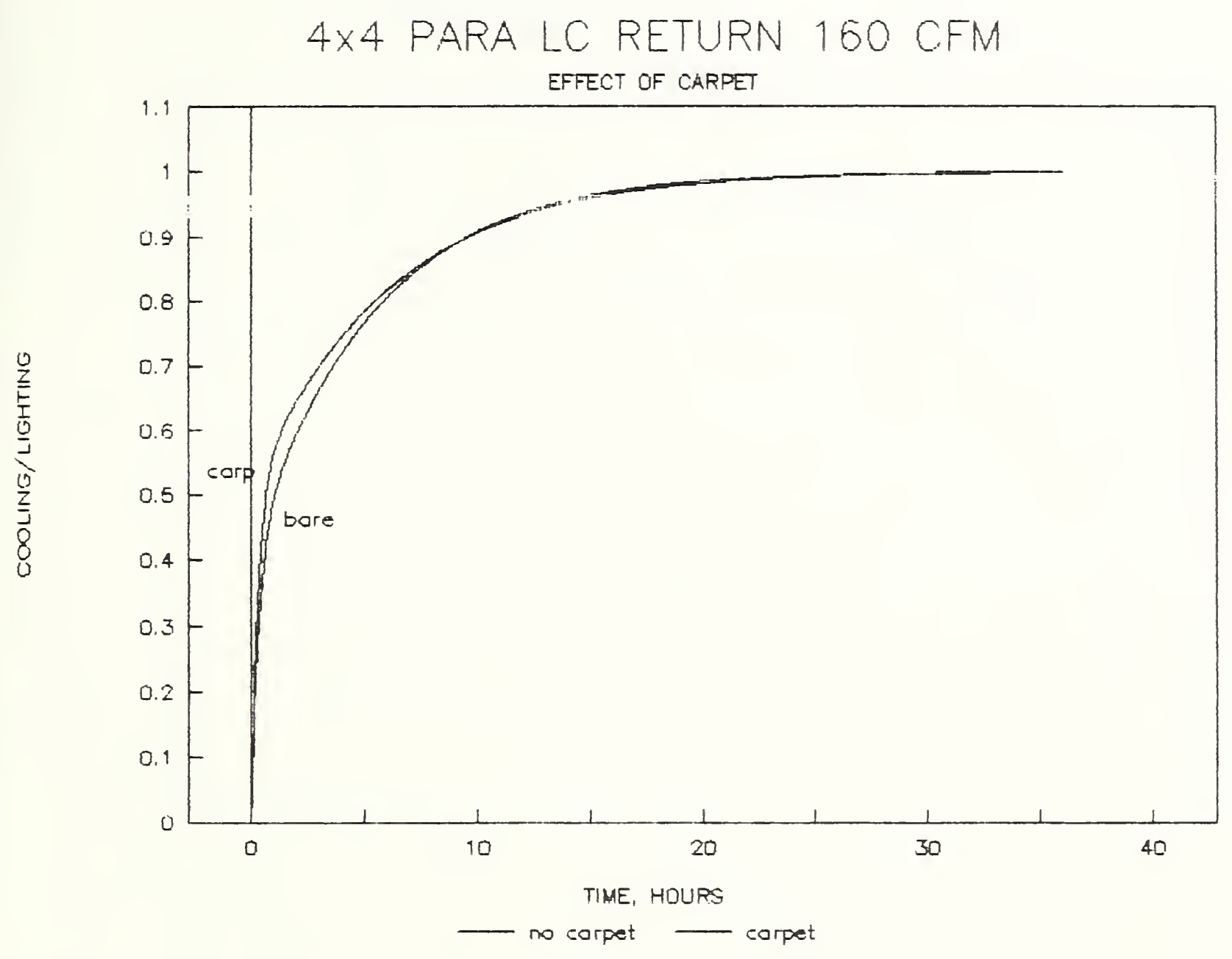

Figure 47. Cooling load regression for the four lamp parabolic diffuser luminaires, $160 \mathrm{cfm}$ airflow, lamp compartment return, with and without carpet 


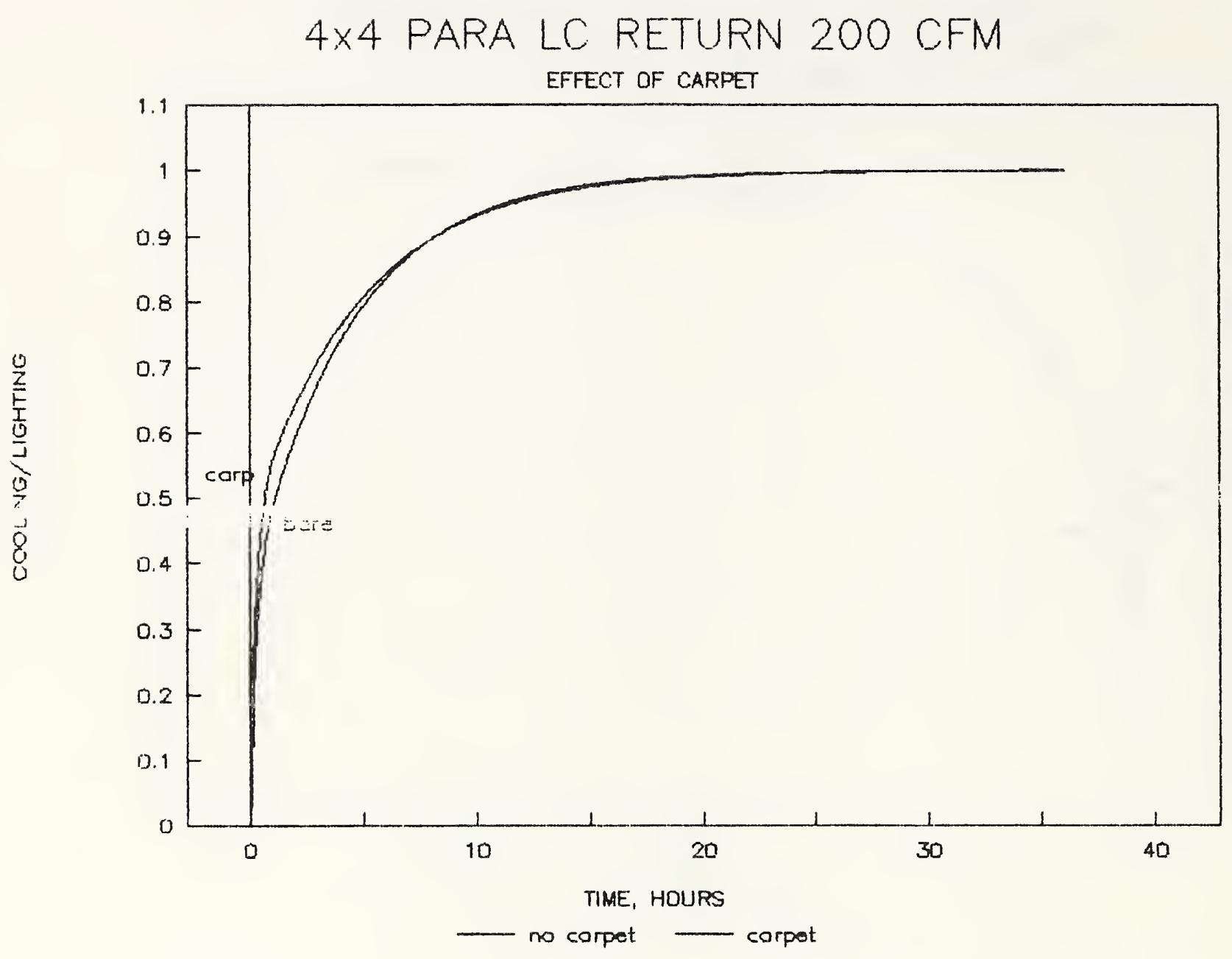

Figure 48. Cooling load regression for the four lamp parabolic diffuser luminaires, $200 \mathrm{cfm}$ airflow, lamp compartment return, with and without carpet 


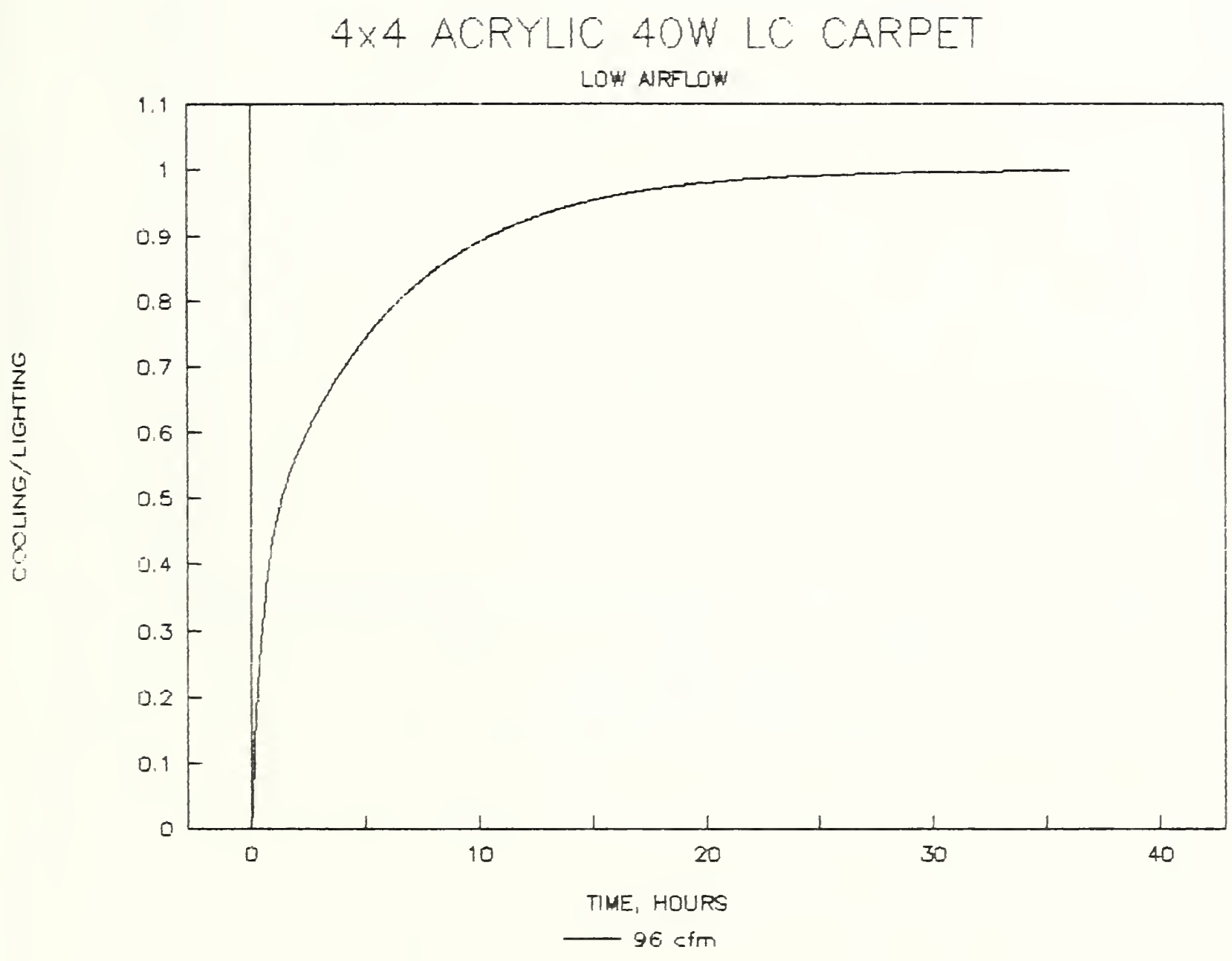

Figure 49. Cooling load regression for the four lamp acrylic lens luminaires, low air flow (96 cfm), lamp compartment return, carpet 


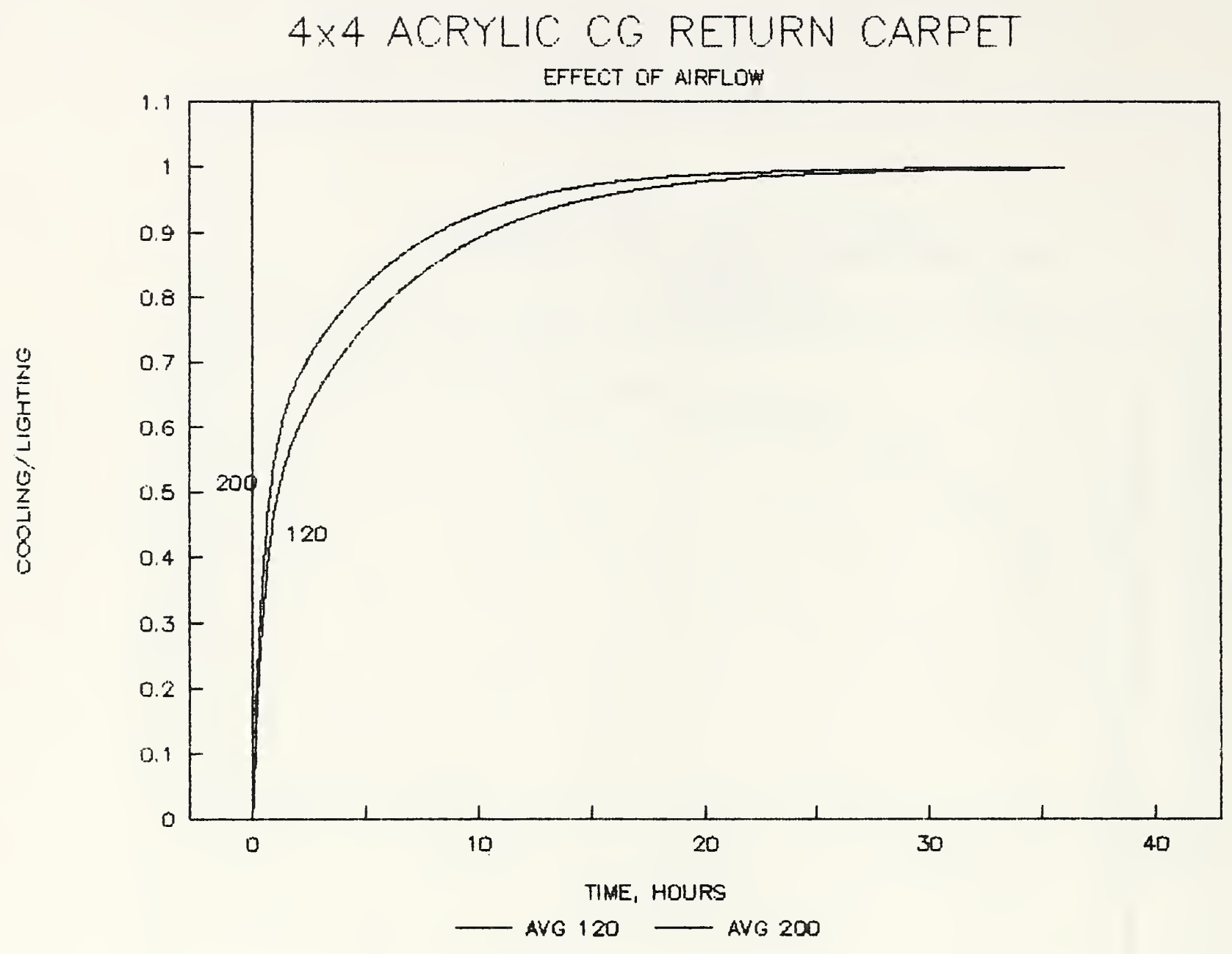

Figure 50. Cooling load regression for the four lamp acrylic lens luminaires, 120 and $200 \mathrm{cfm}$ air flow, ceiling grill return, carpet 


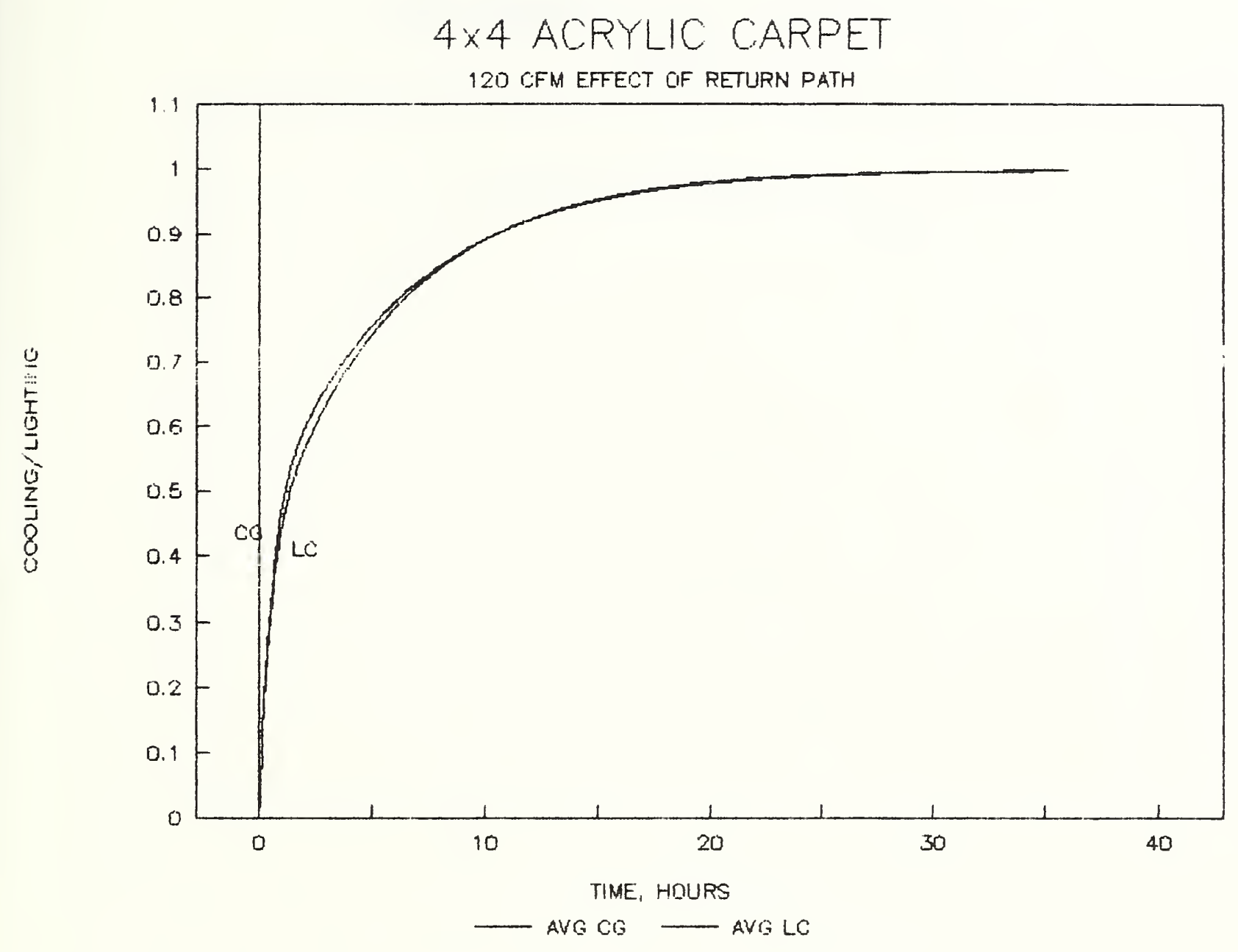

Figure 51. Cooling load regression for the four lamp acrylic lens luminaires, $120 \mathrm{cfm}$ air flow, ceiling grill and lamp compartment return, 


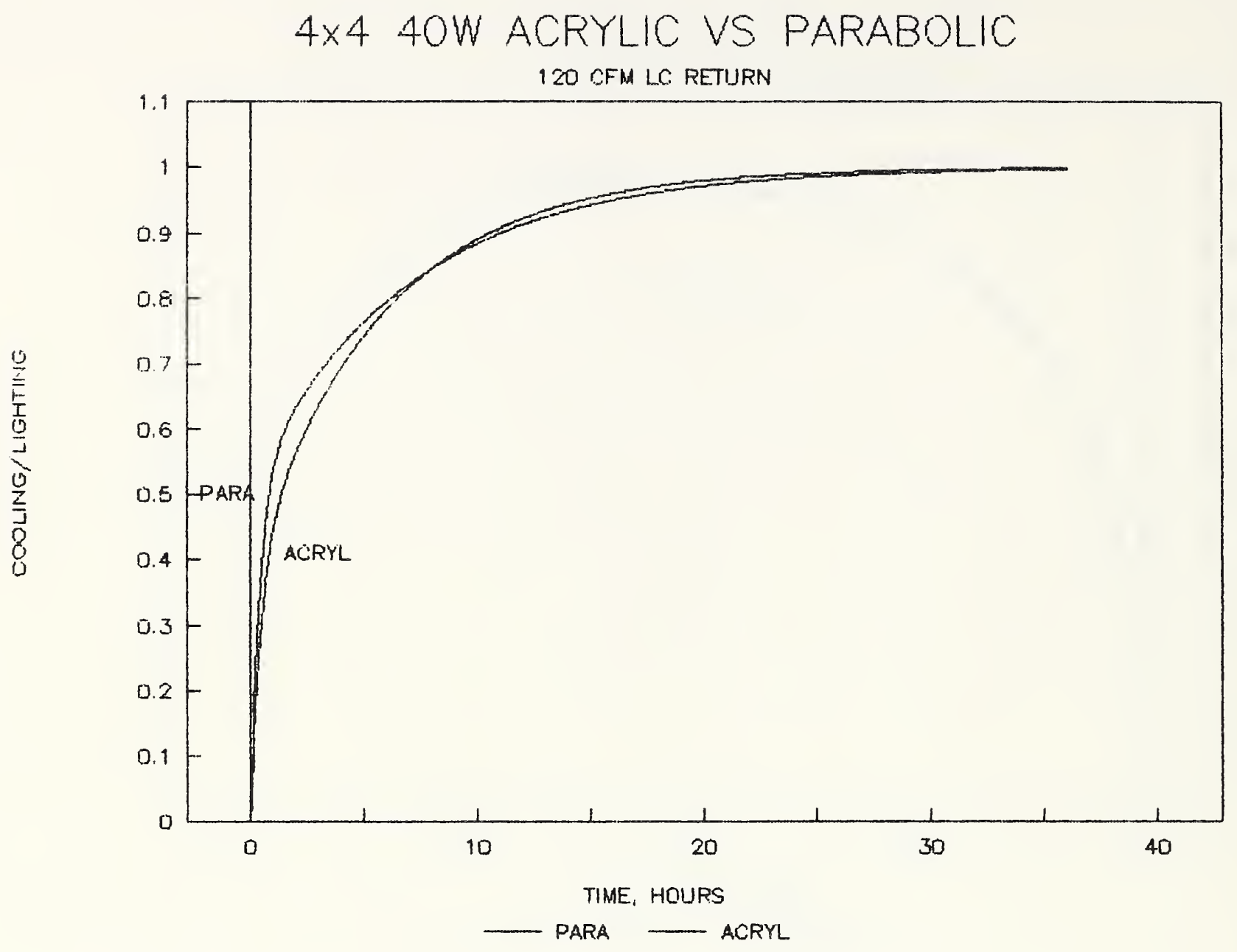

Figure 52. Cooling load regression for the four lamp acrylic lens and parabolic diffuser luminaires, $120 \mathrm{cfm}$ air flow, lamp compartment return 


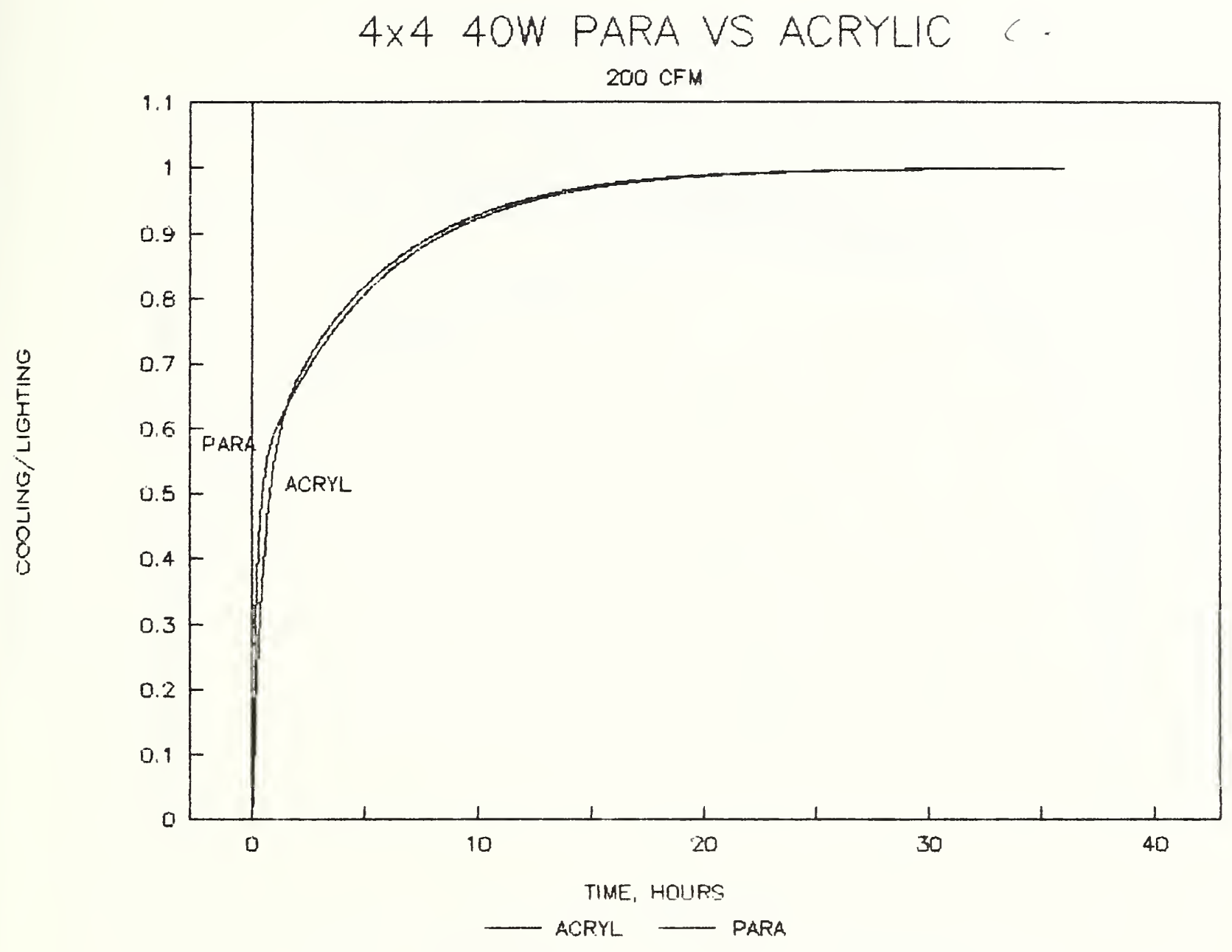

Figure 53. Cooling load regression for the four lamp acrylic lens and parabolic diffuser luminaires, $200 \mathrm{cfm}$ air flow, ceiling grill return 


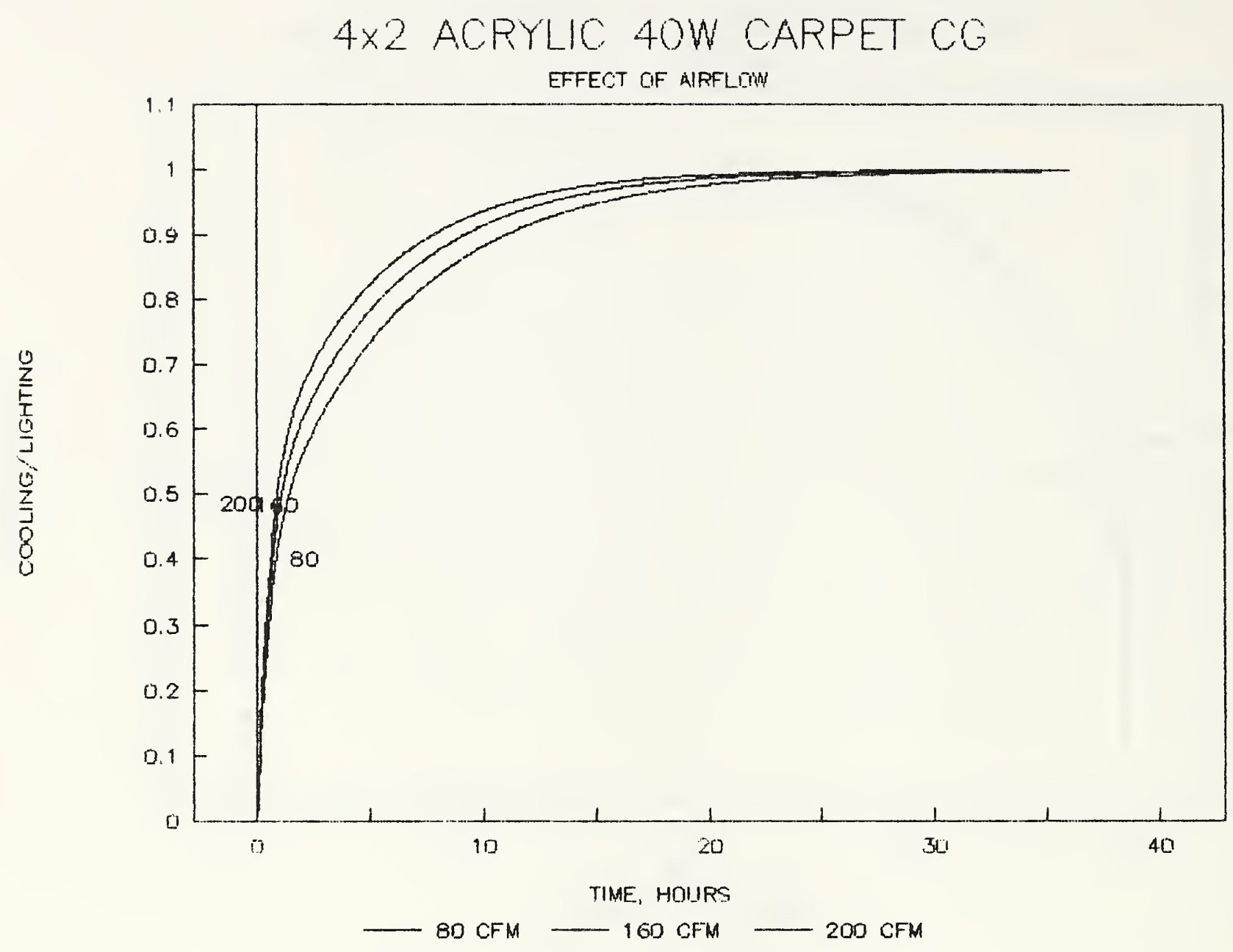

Figure 54. Cooling load regression for four two-lamp acrylic lens luminaires, ceiling grill return, carpet, effect of air flow ceiling grill return 


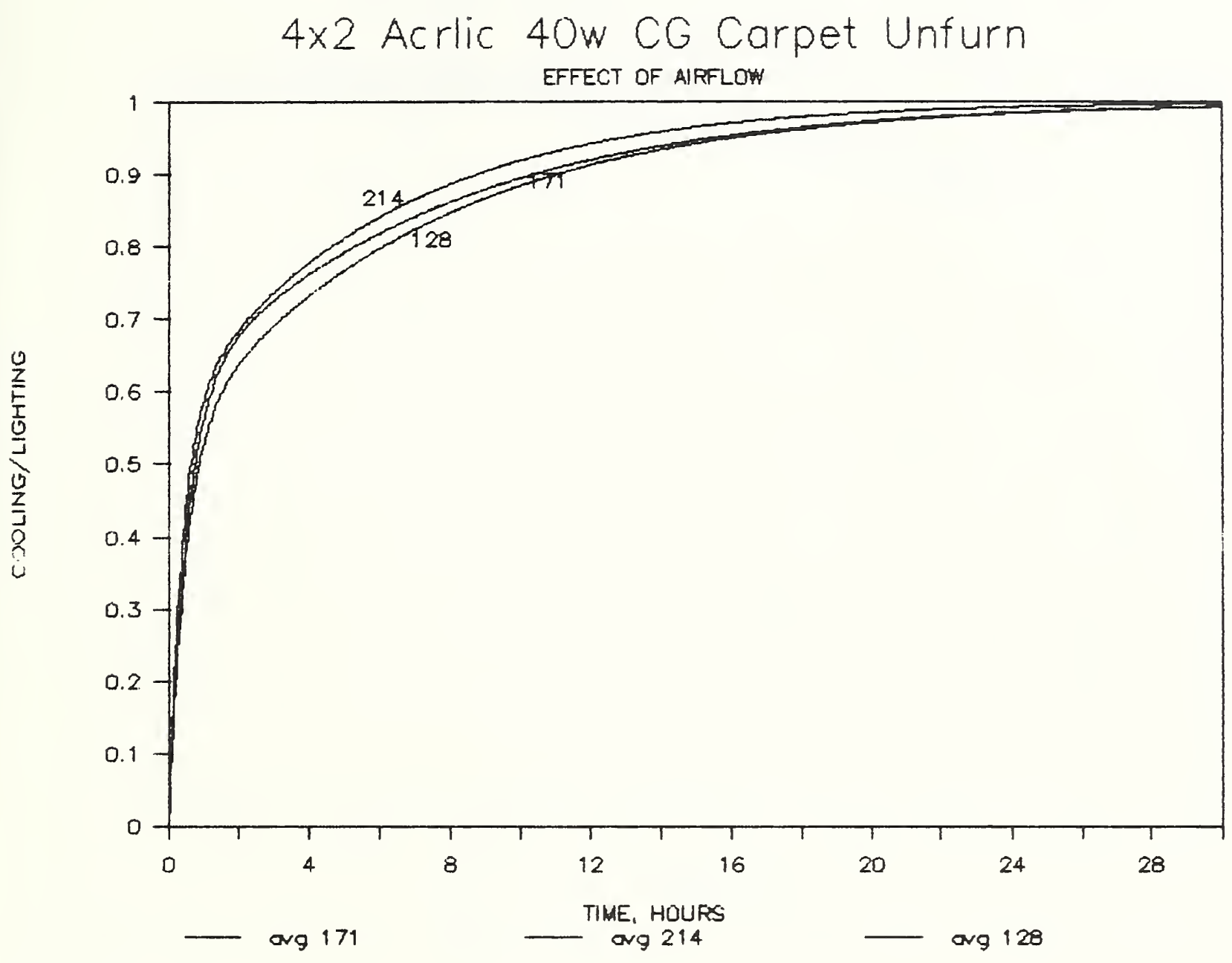

Figure 55. Cooling load regression for four two-lamp acrylic lens luminaires, ceiling grill return, carpet, effect of air flow 


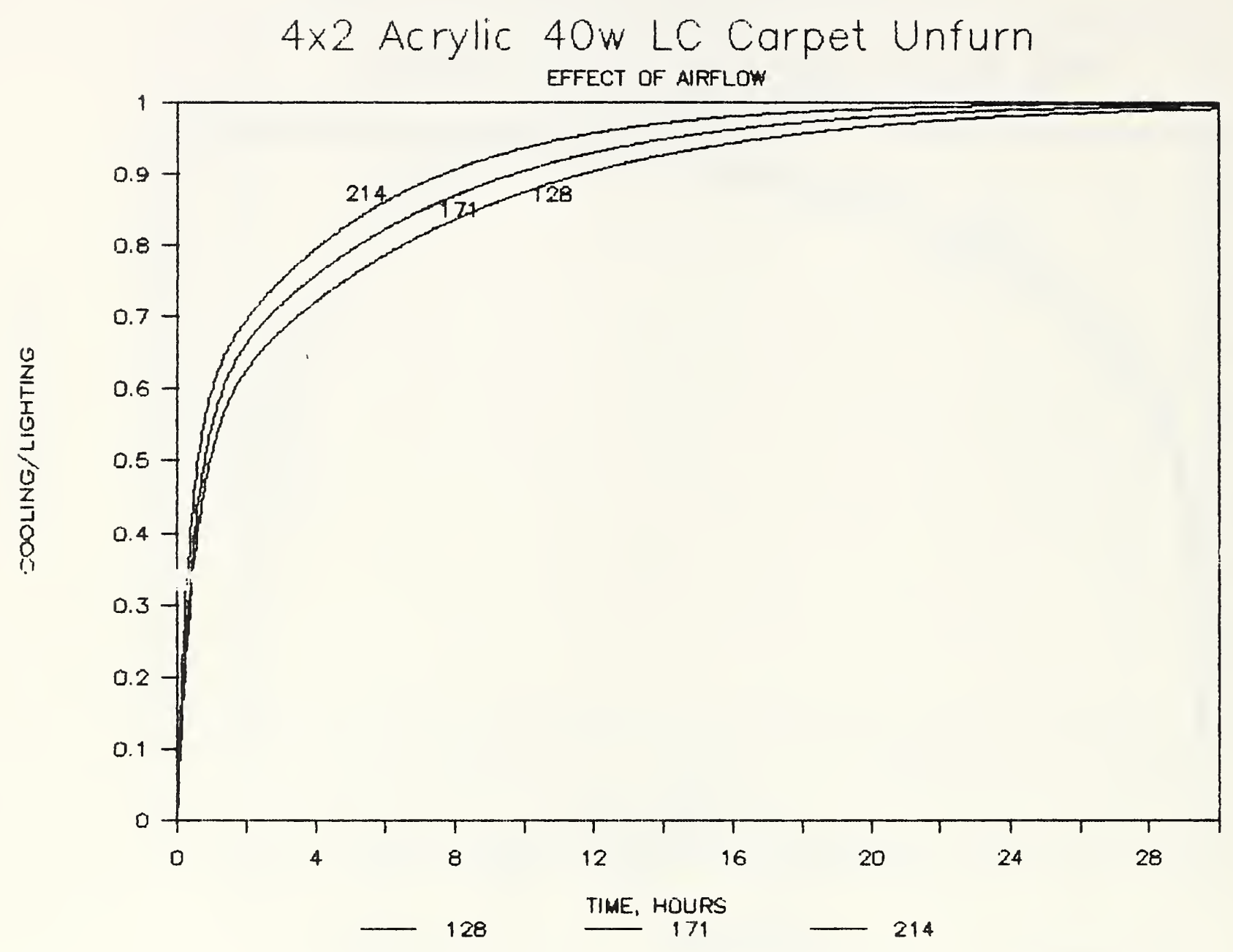

Figure 56. Cooling load regression for four two-lamp acrylic lens luminaires, lamp compartment return, carpet, effect of air flow 


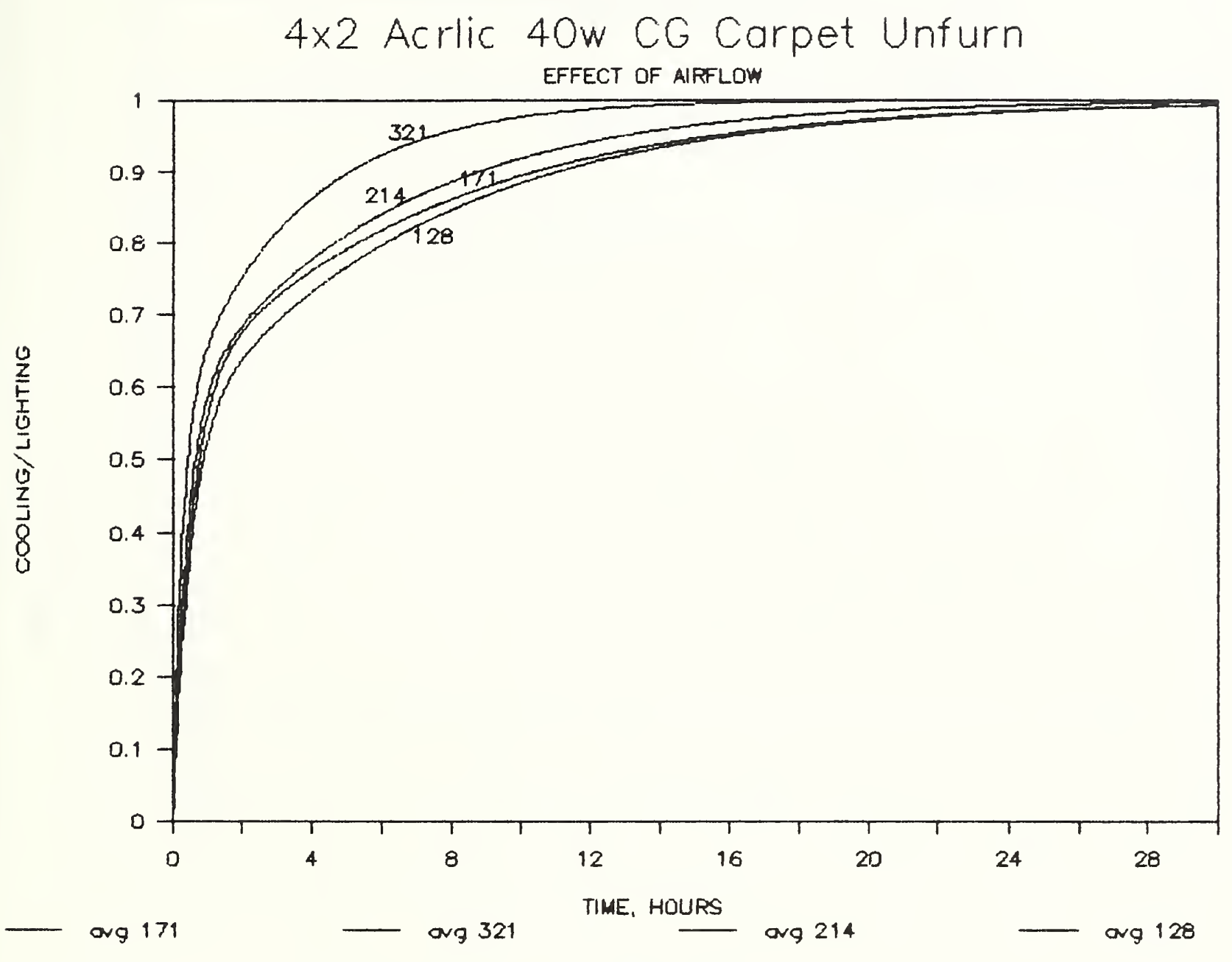

Figure 57. Cooling load regression for four two-lamp acrylic lens luminaires, ceiling grill return, carpet, unfurnished 


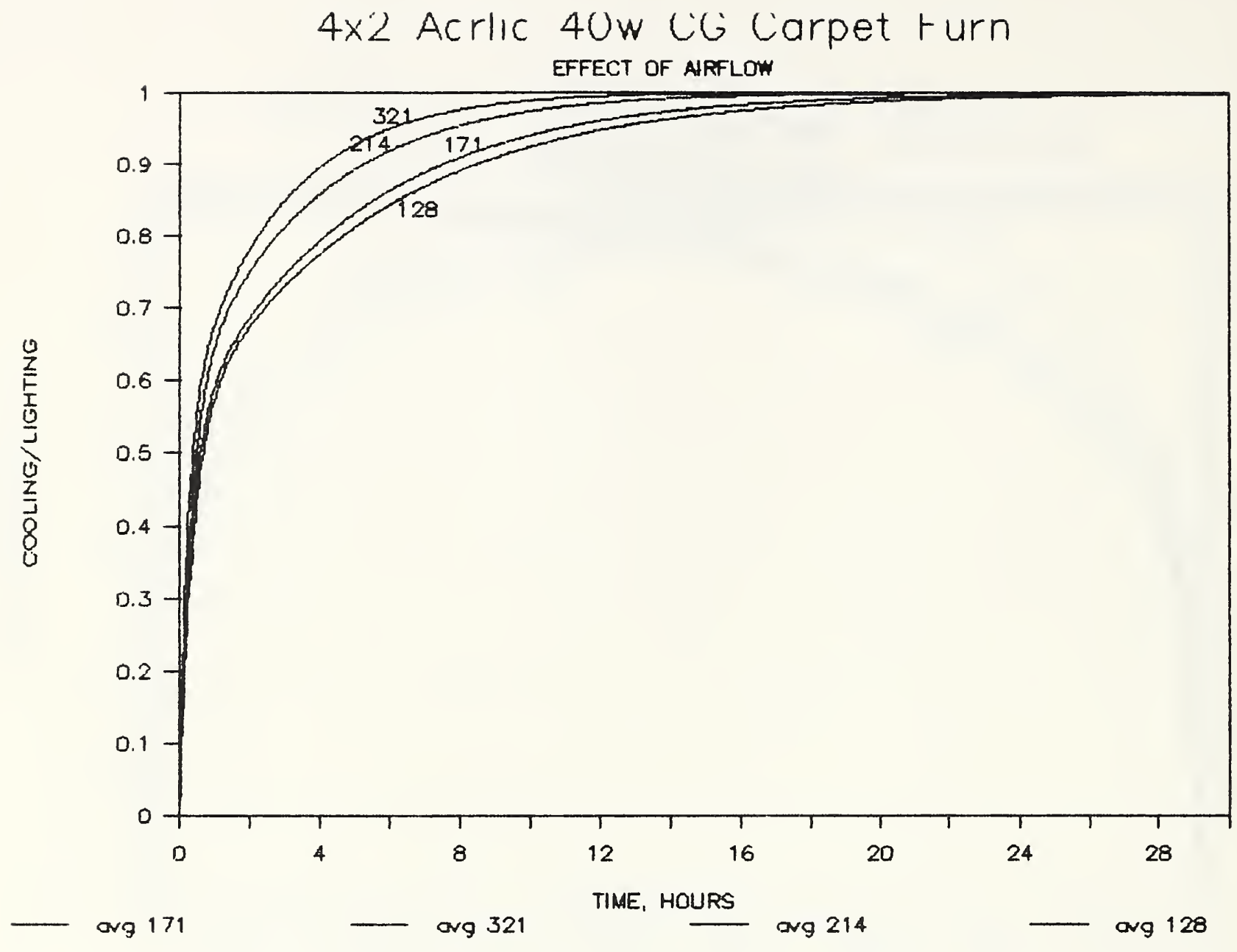

Figure 58. Cooling load regression for four two-lamp acrylic lens luminaires, ceiling grill return, carpet, furnished 


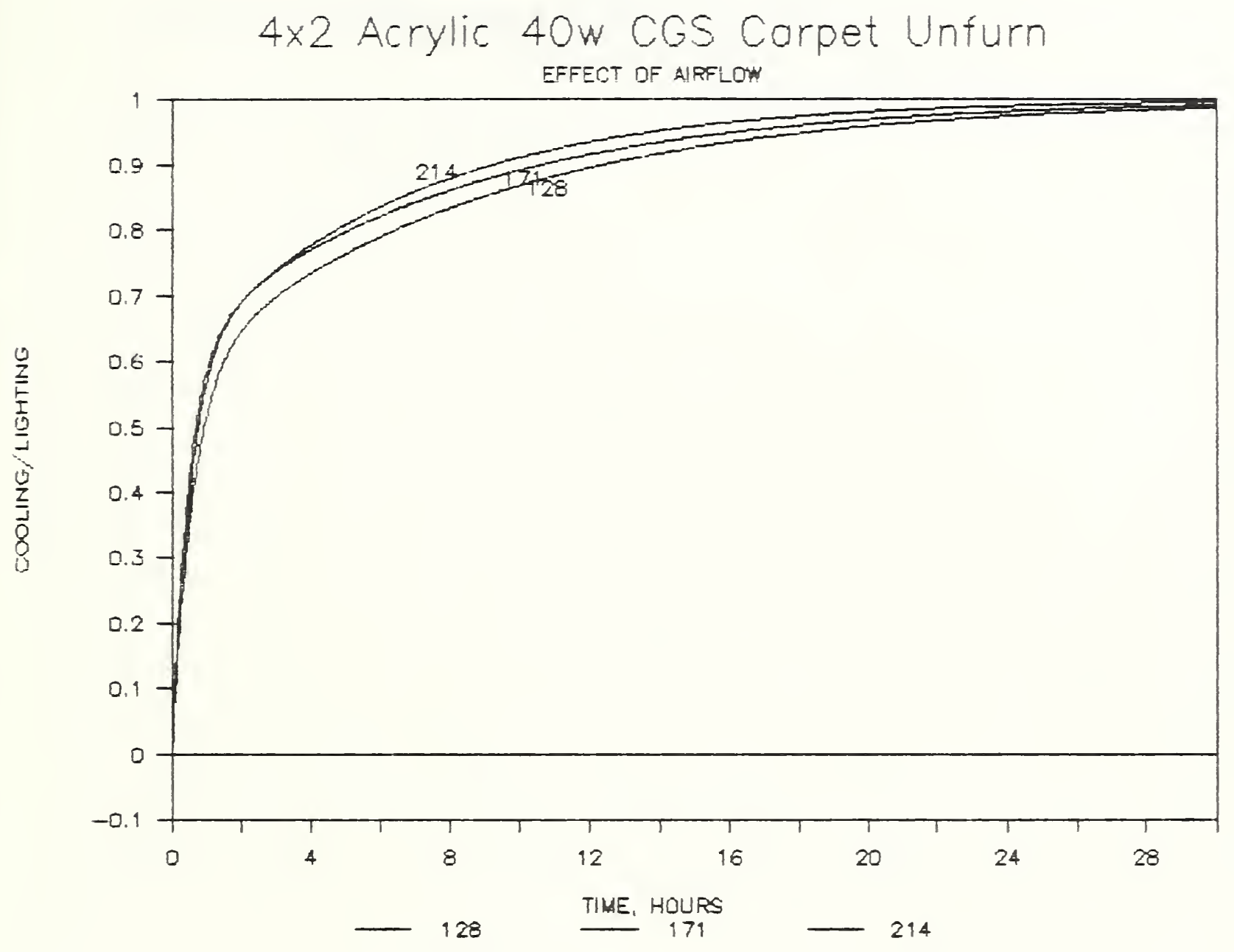

Figure 59. Cooling load regression for four two-lamp acrylic lens luminaires, sealed ceiling grill return, carpet, unfurnished 


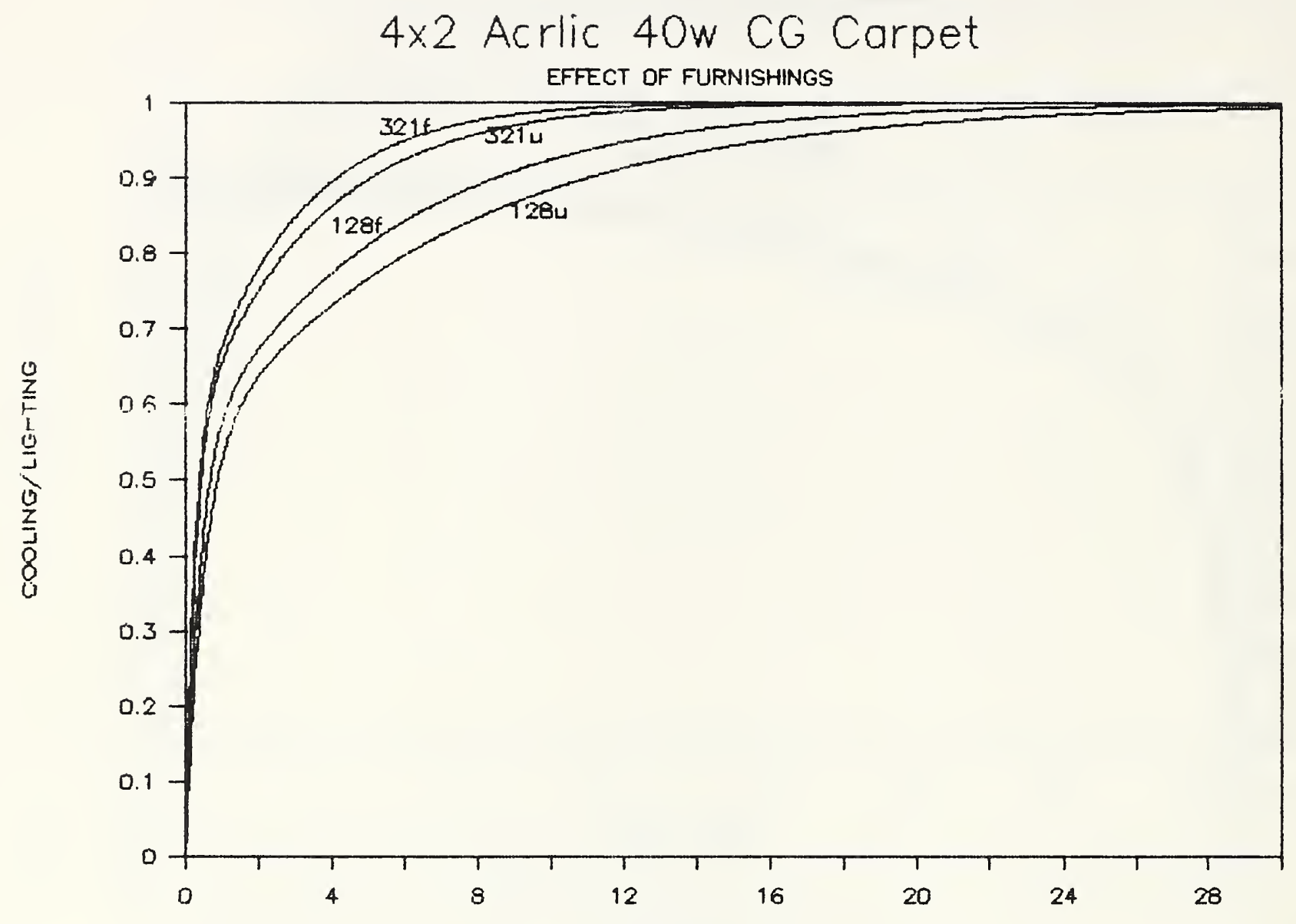

ovg $128 u$

ovg $321 u$

TIME, HOURS

- ovg $321 f$

- ovg $128 f$

Figure 60. Cooling load regression for four two-lamp acrylic lens luminaires, ceiling grill return, carpet, effect of furnishings 


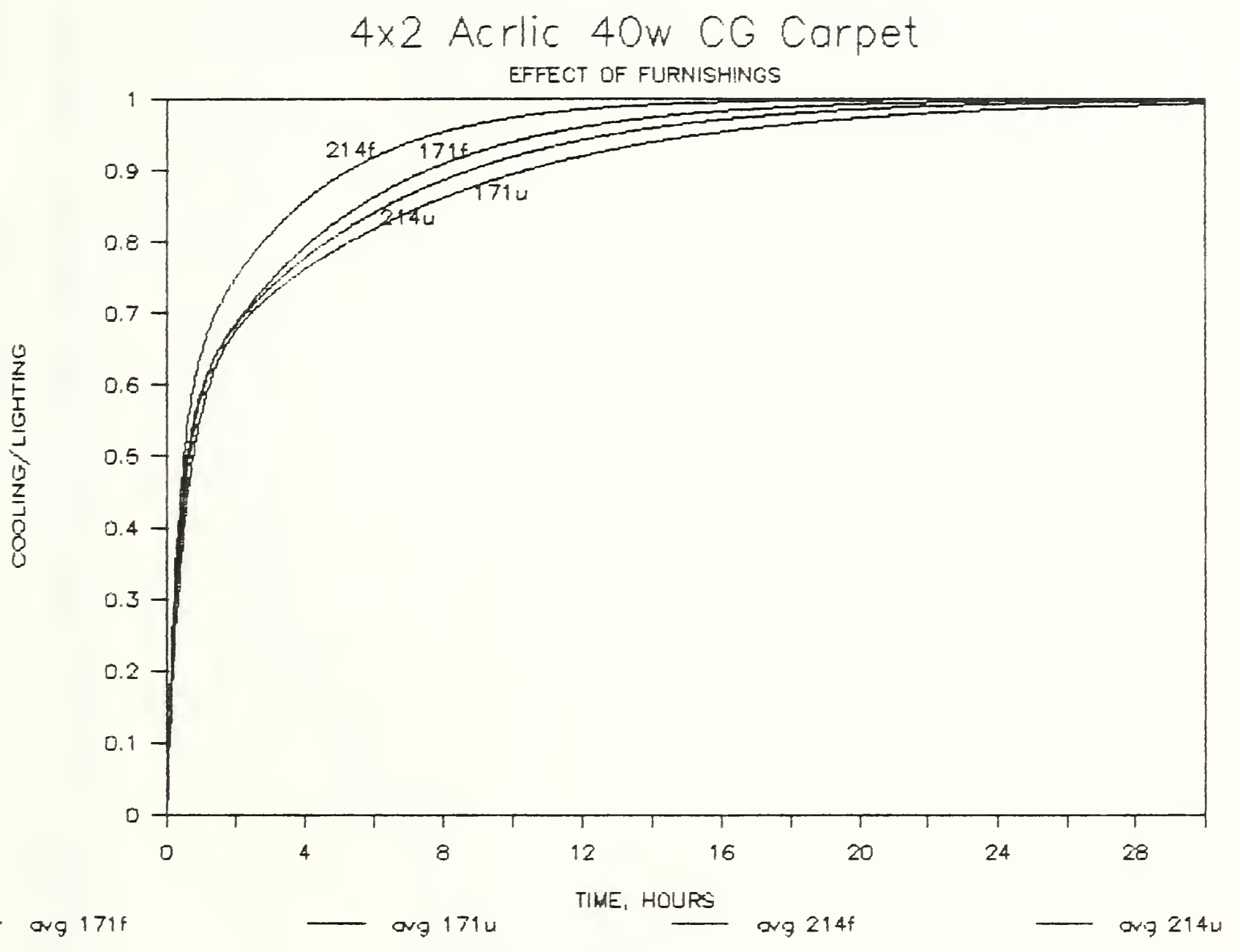

Figure 61. Cooling load regression for four two-lamp acrylic lens luminaires, ceiling grill return, carpet, effect of furnishings 


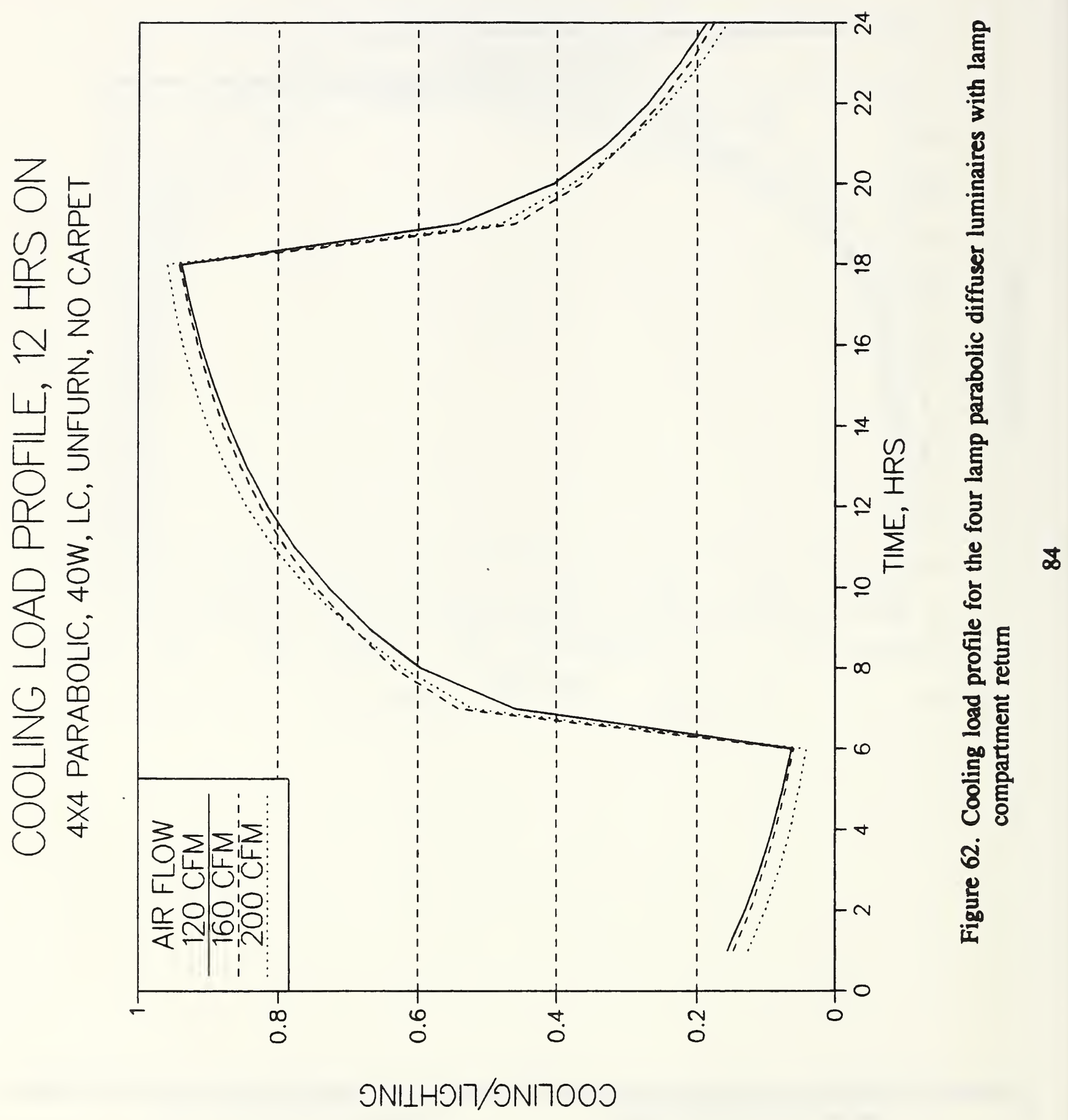




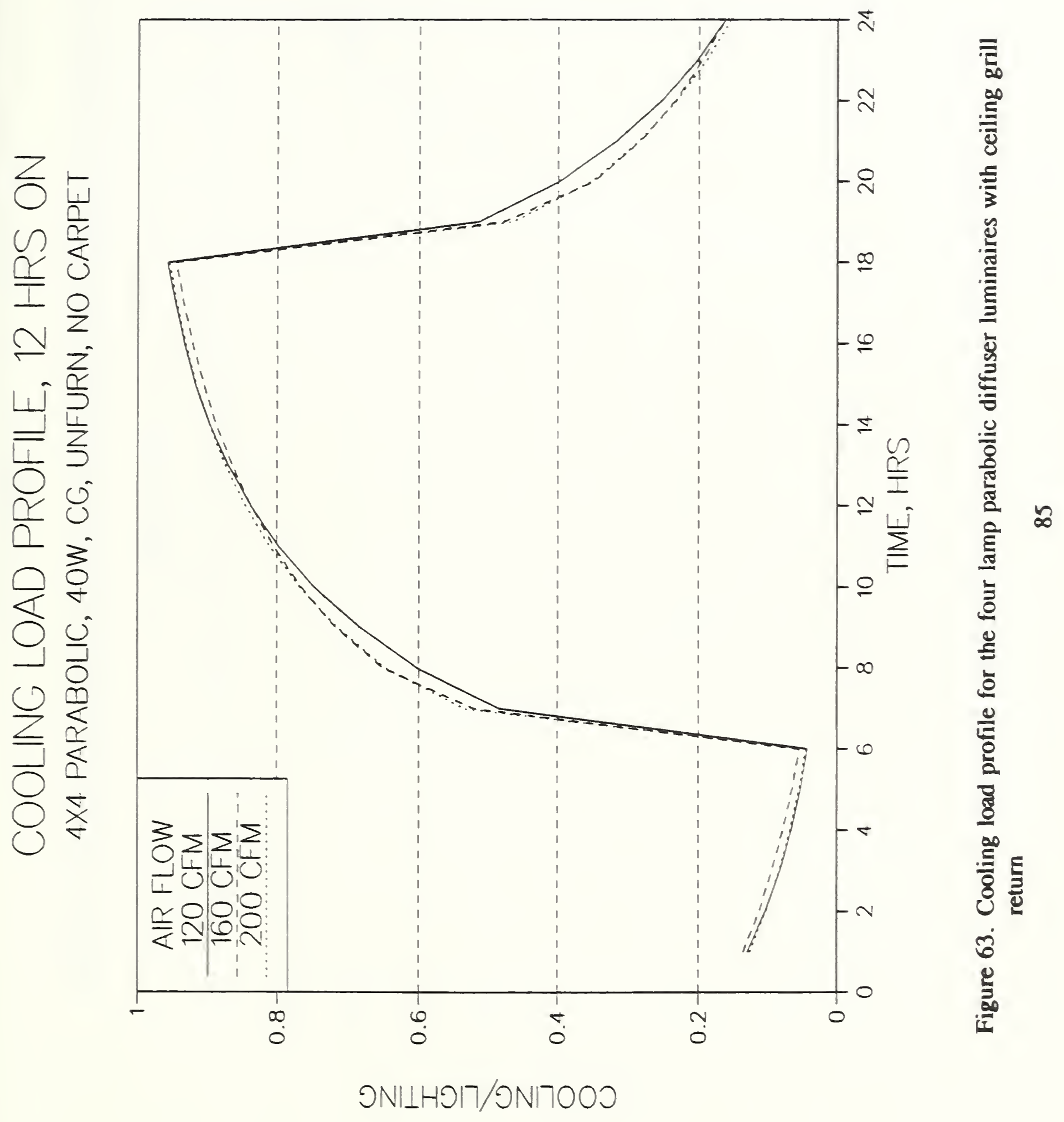




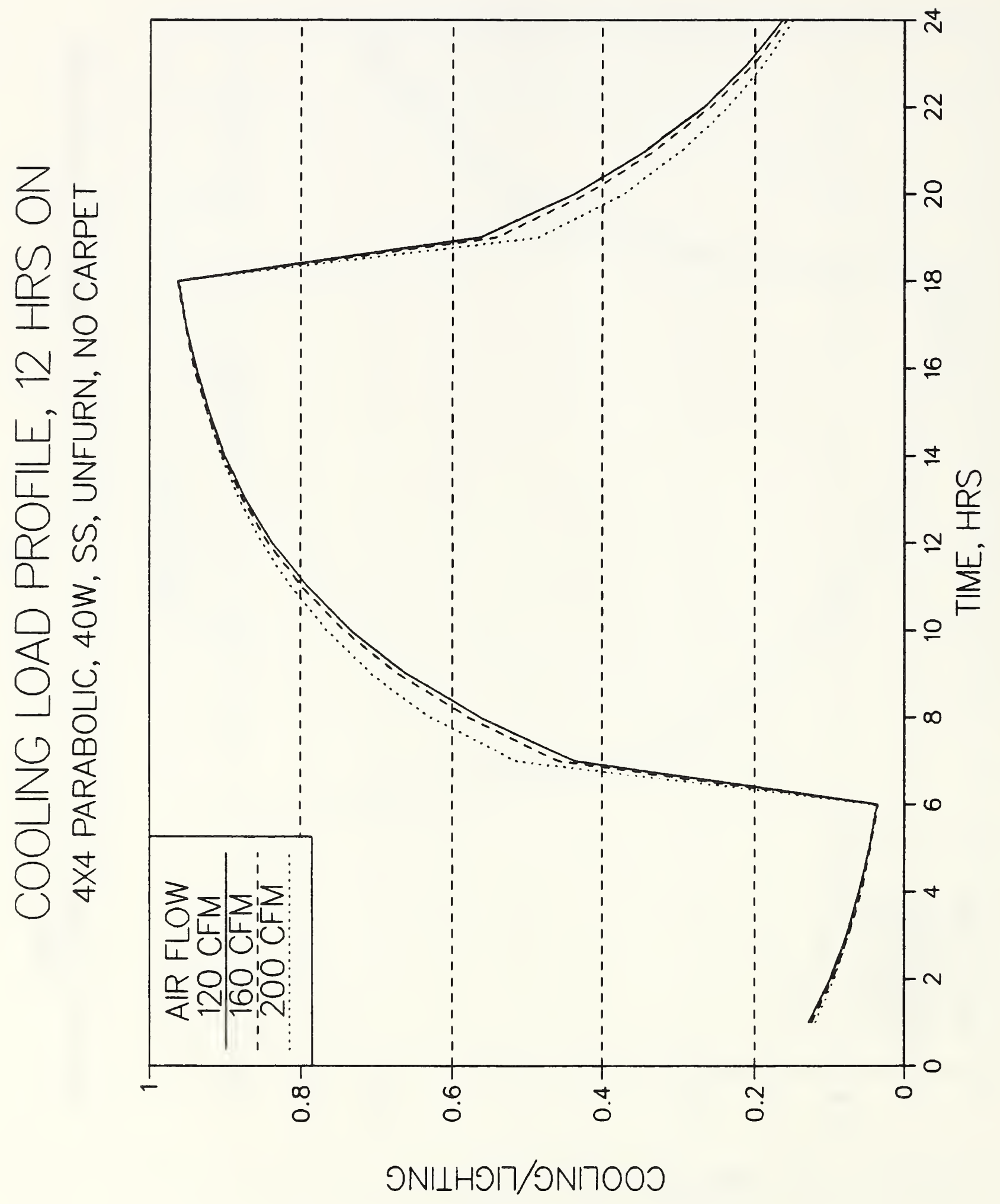

톨

유 


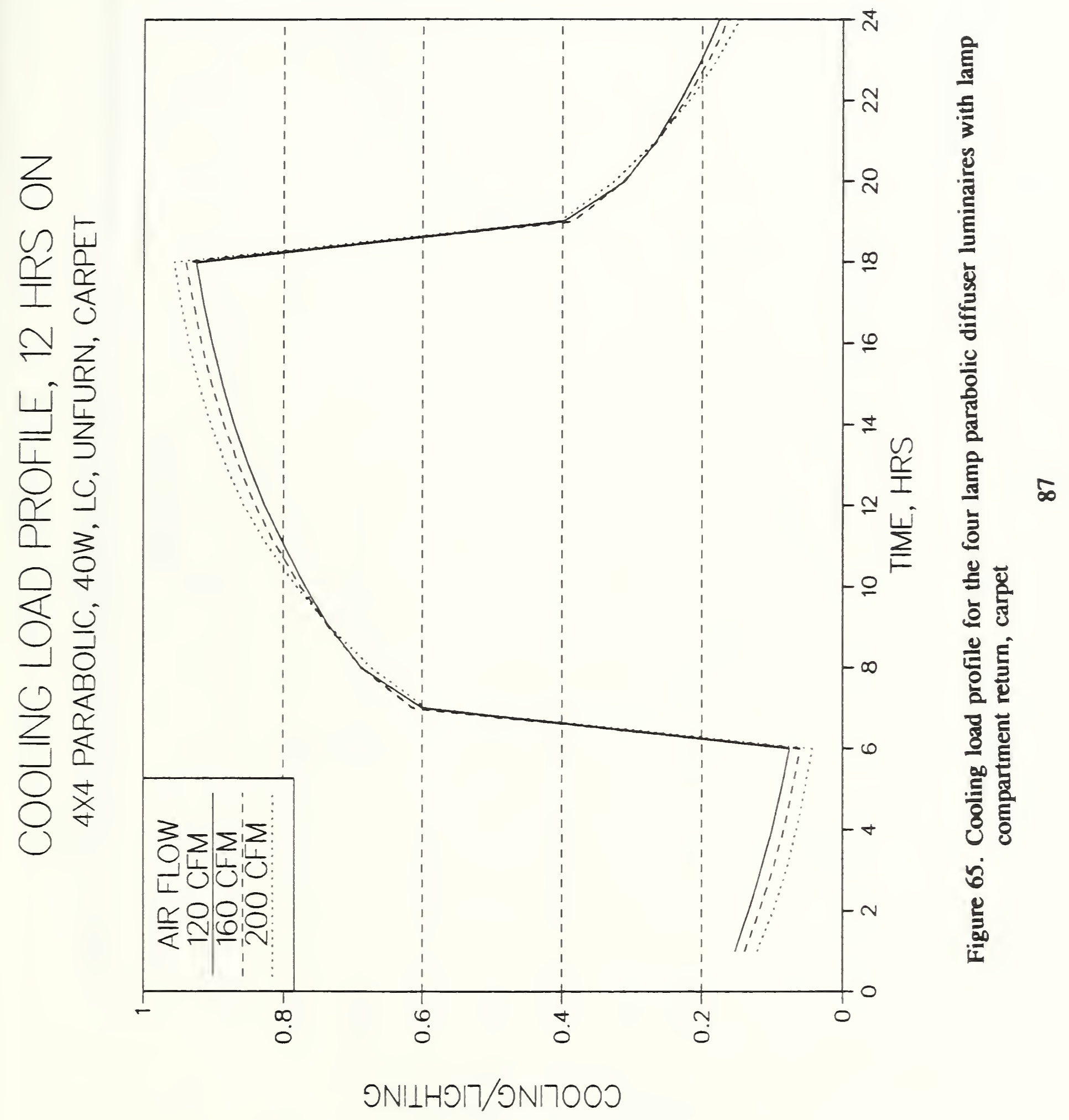




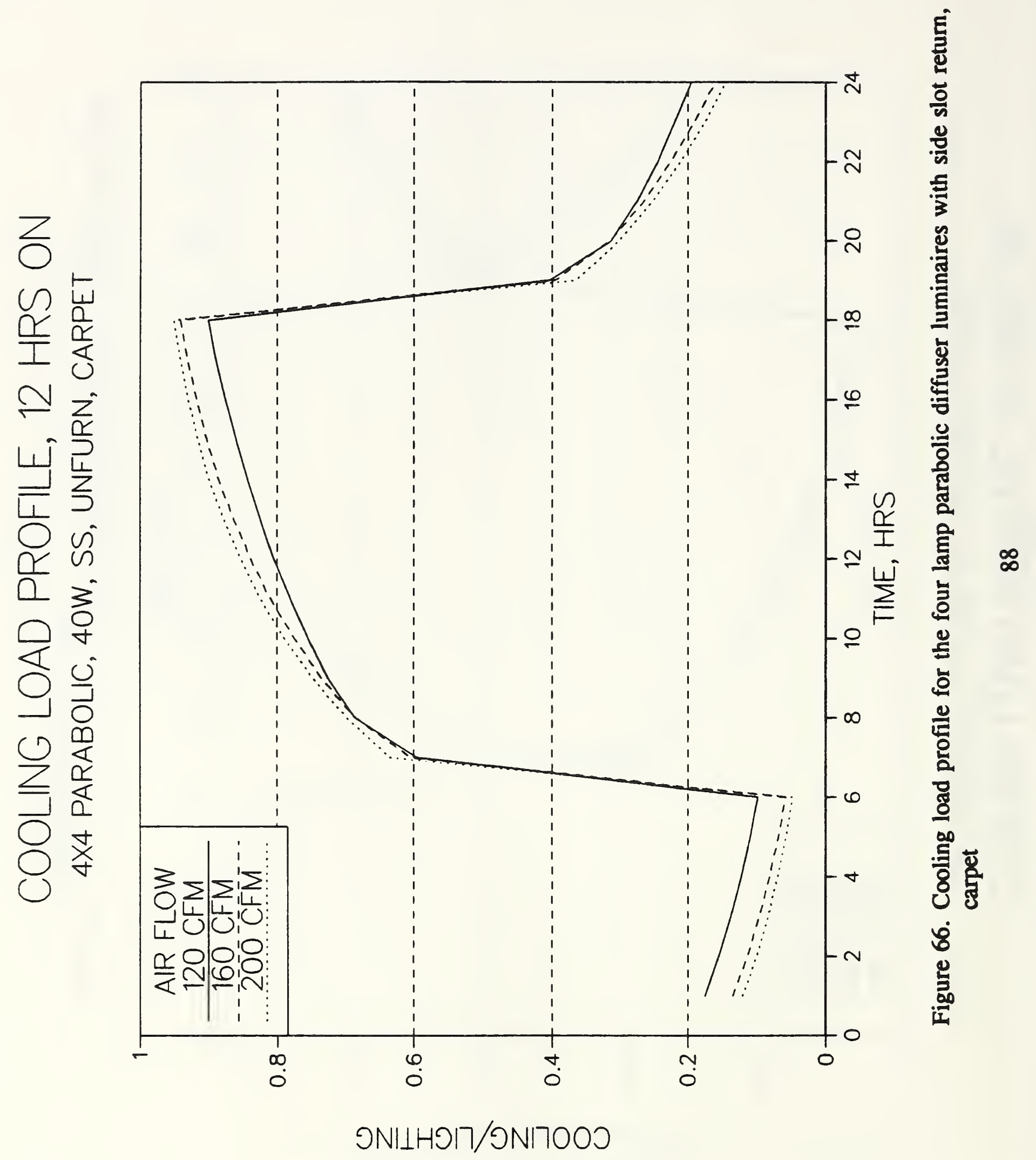




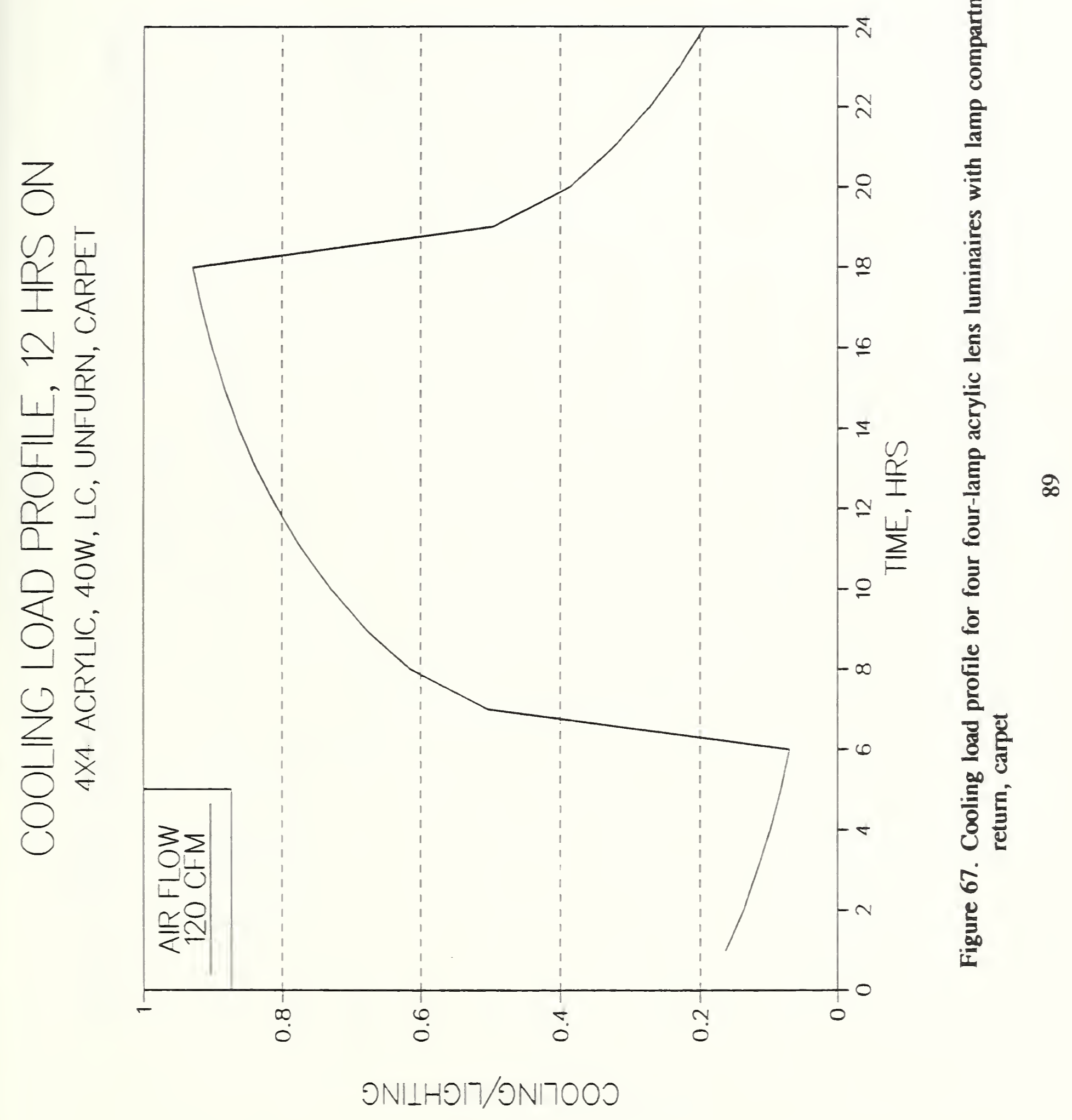




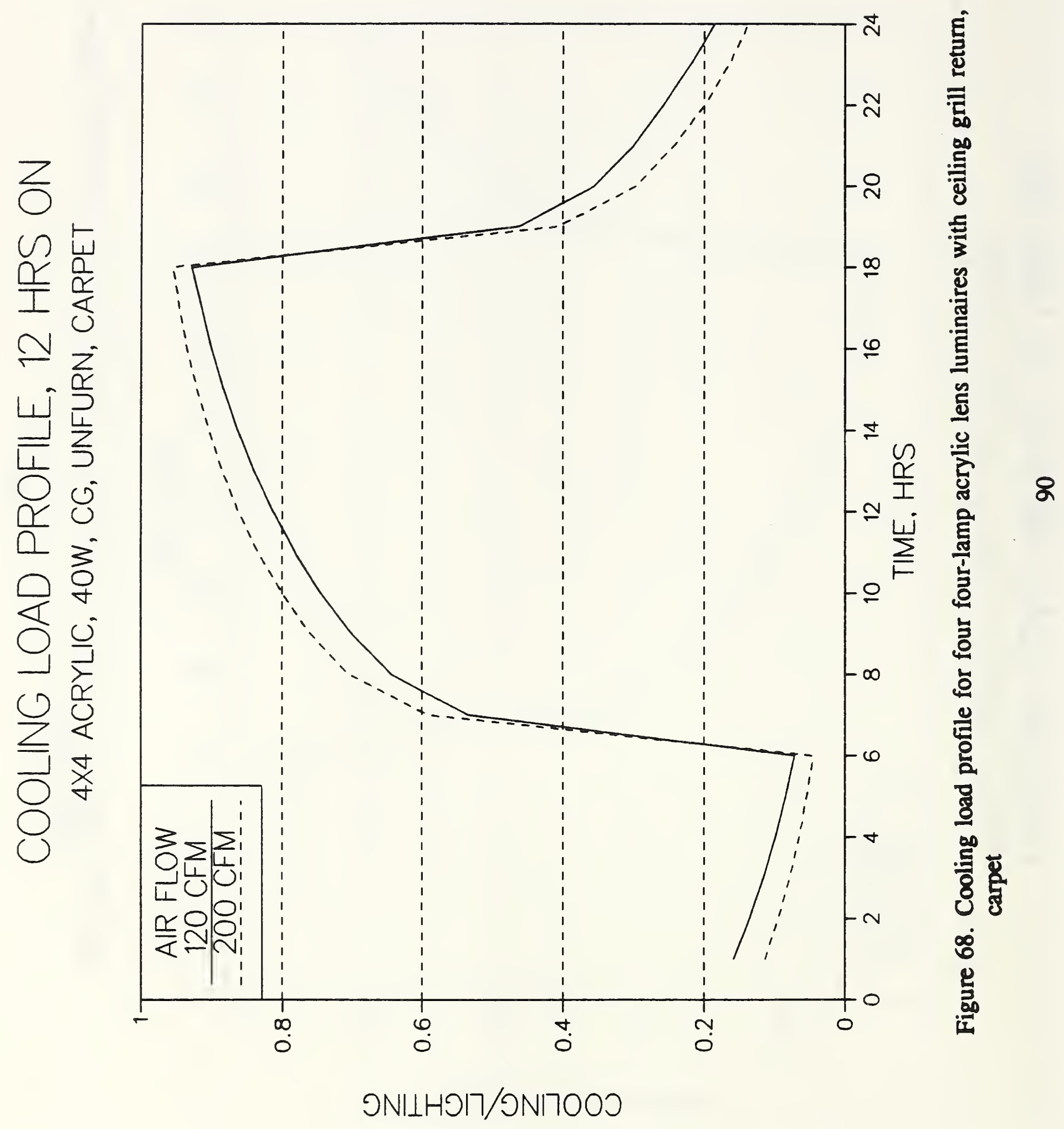




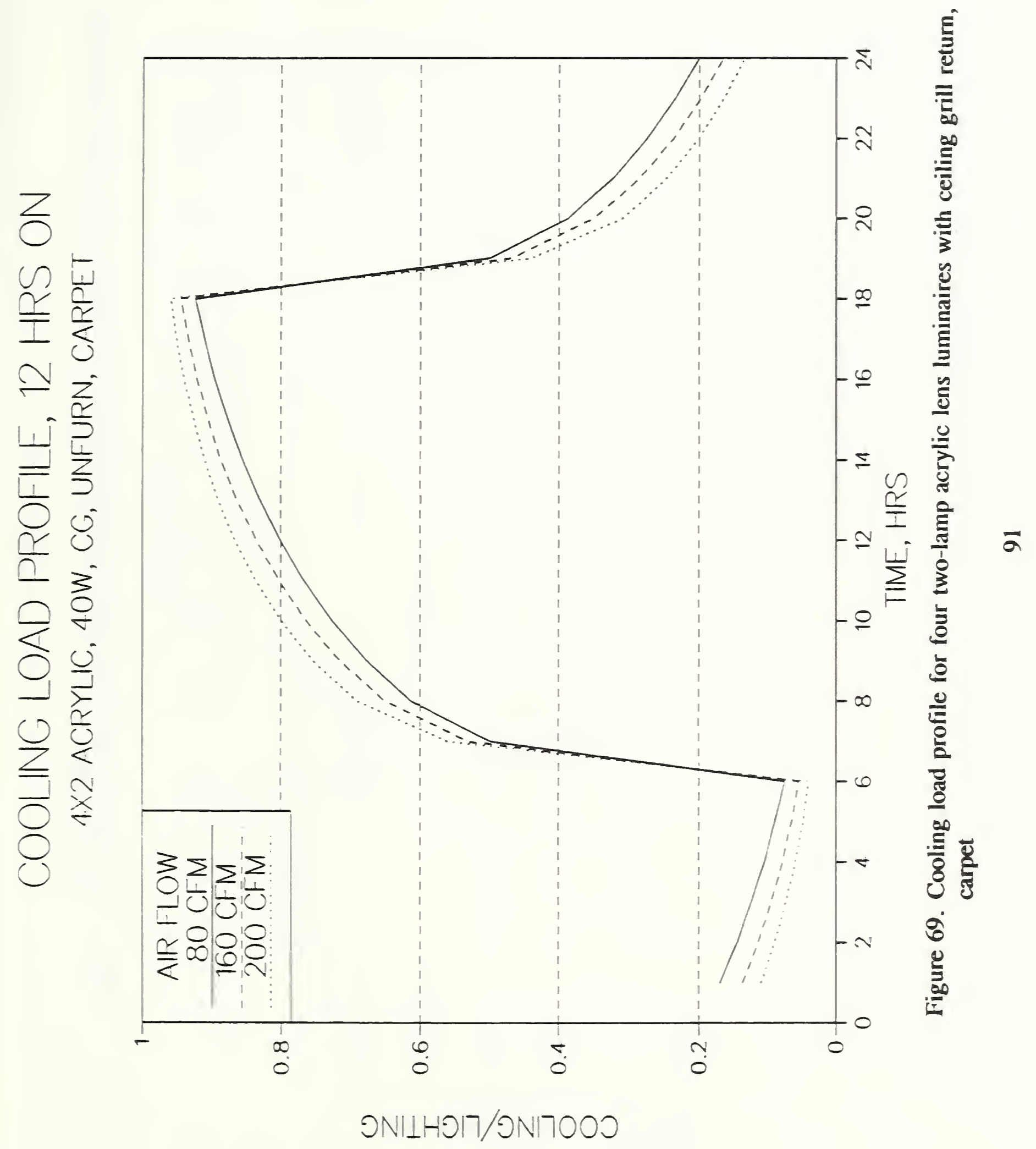




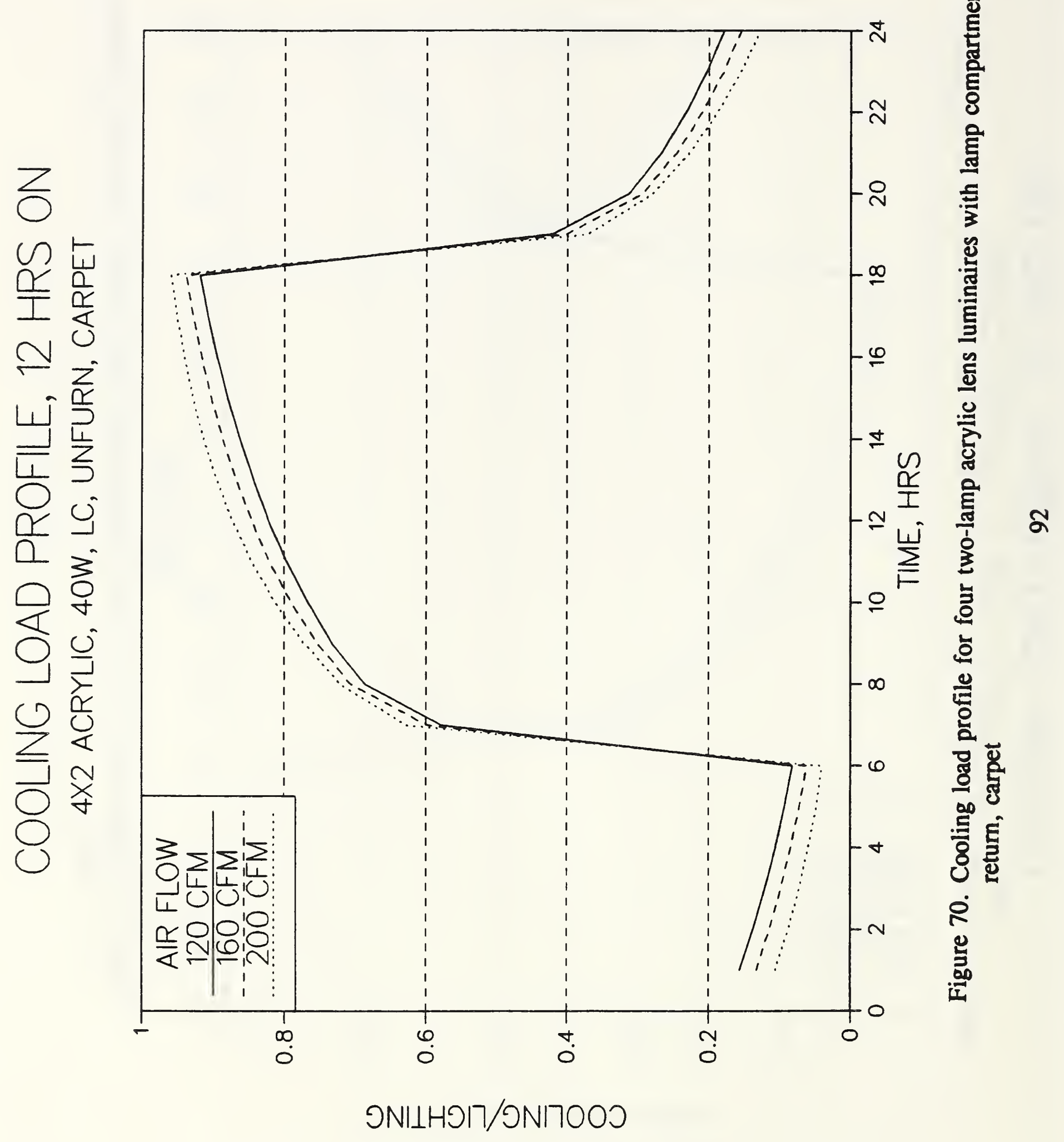




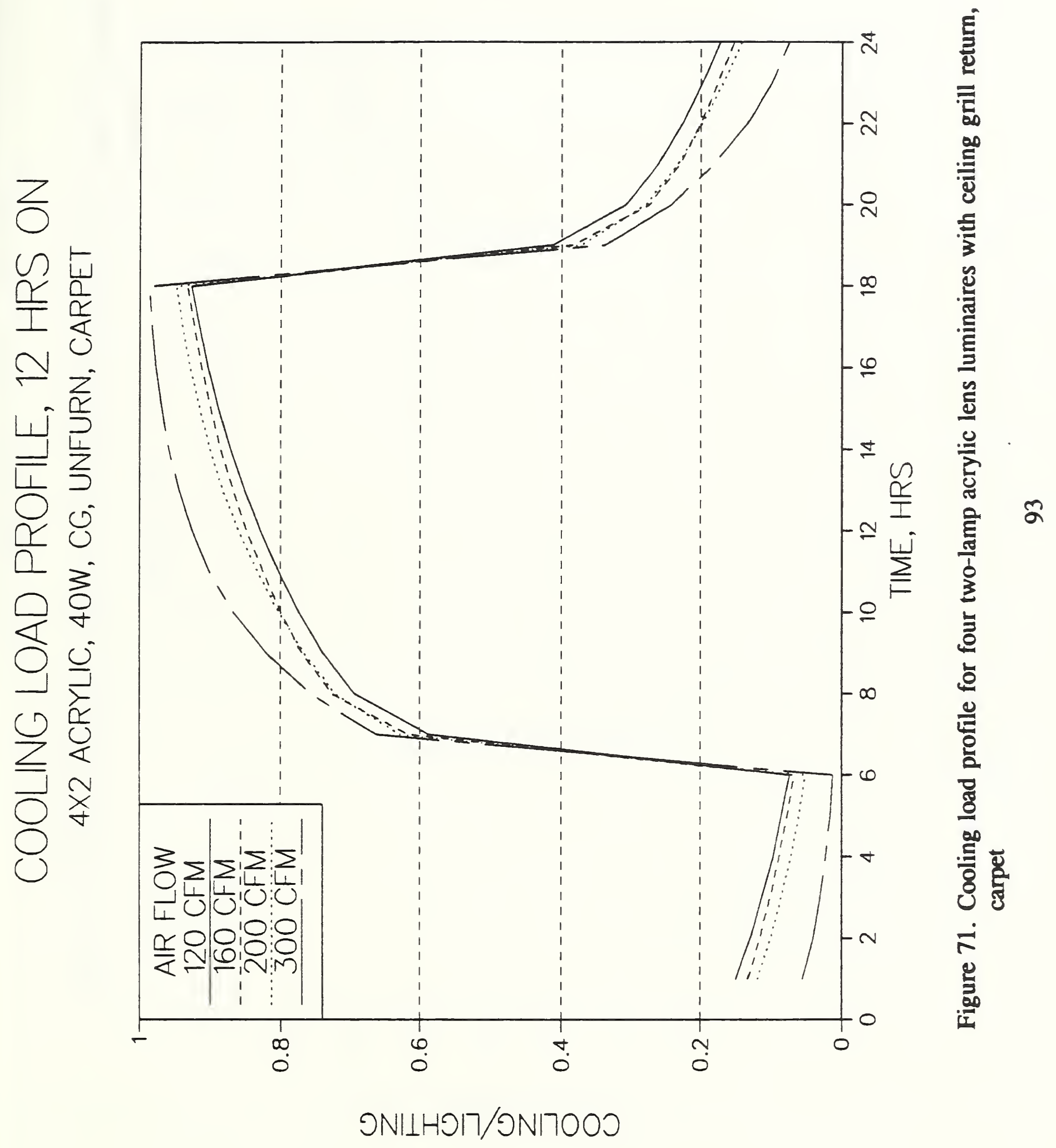




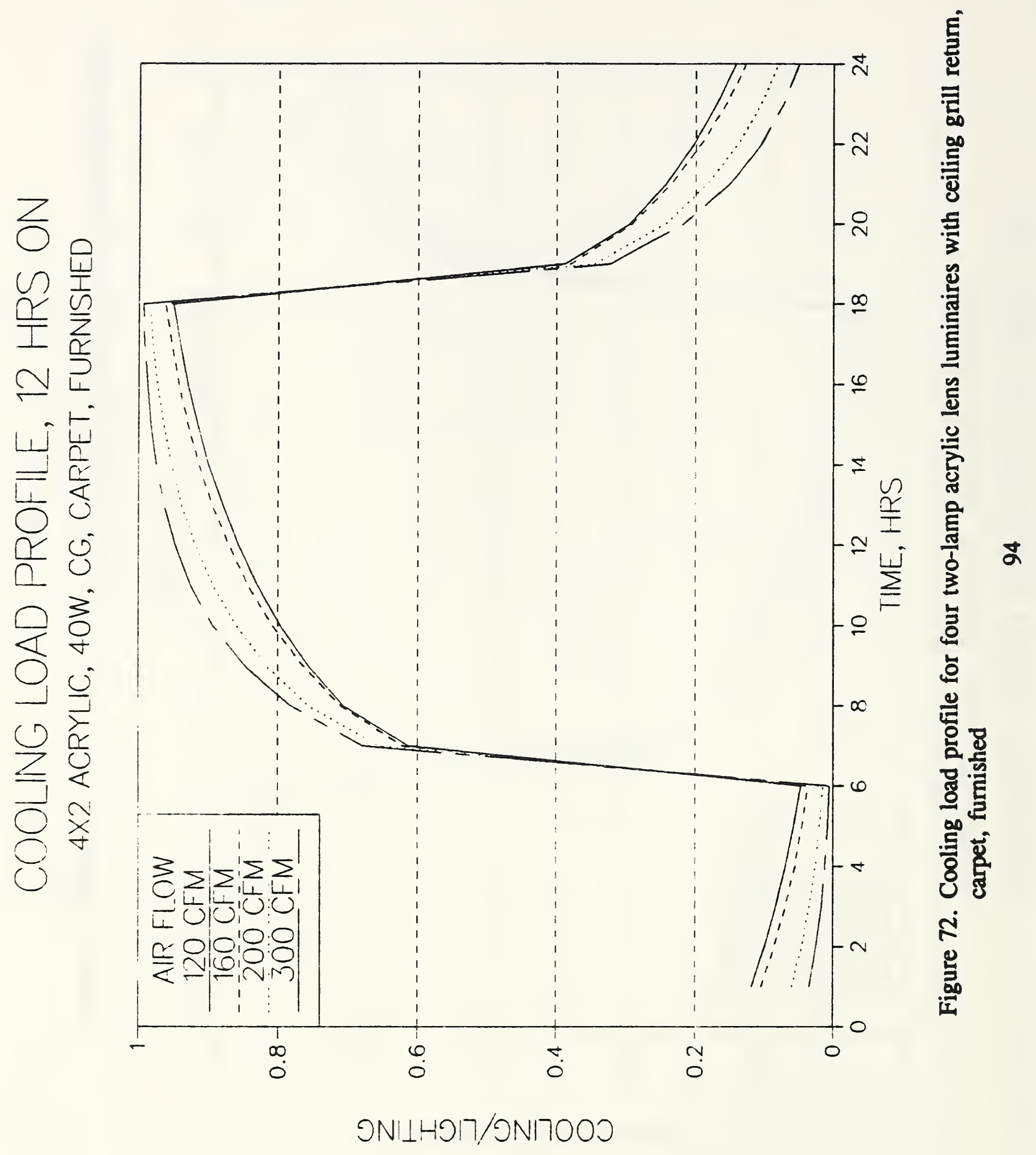




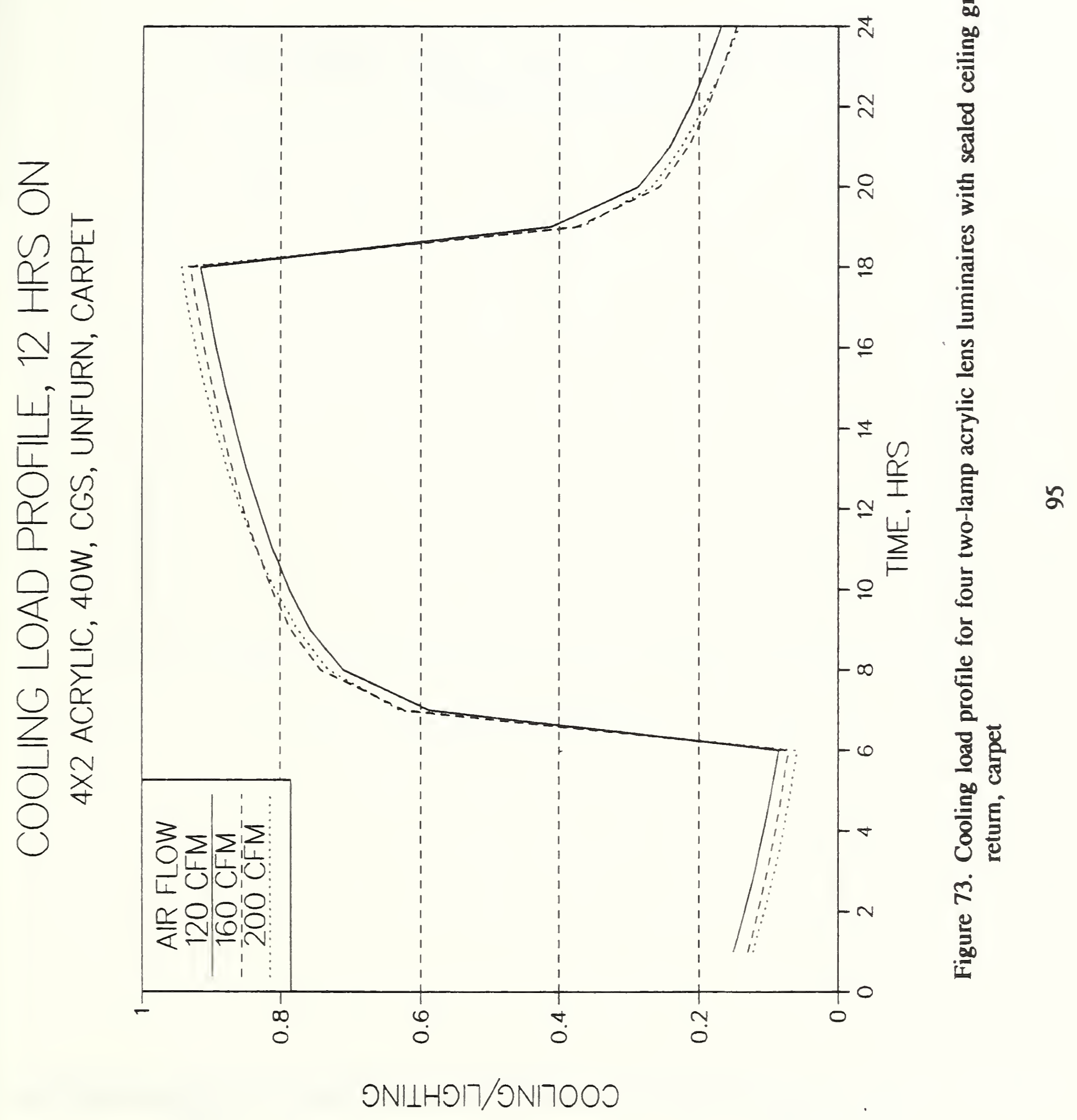




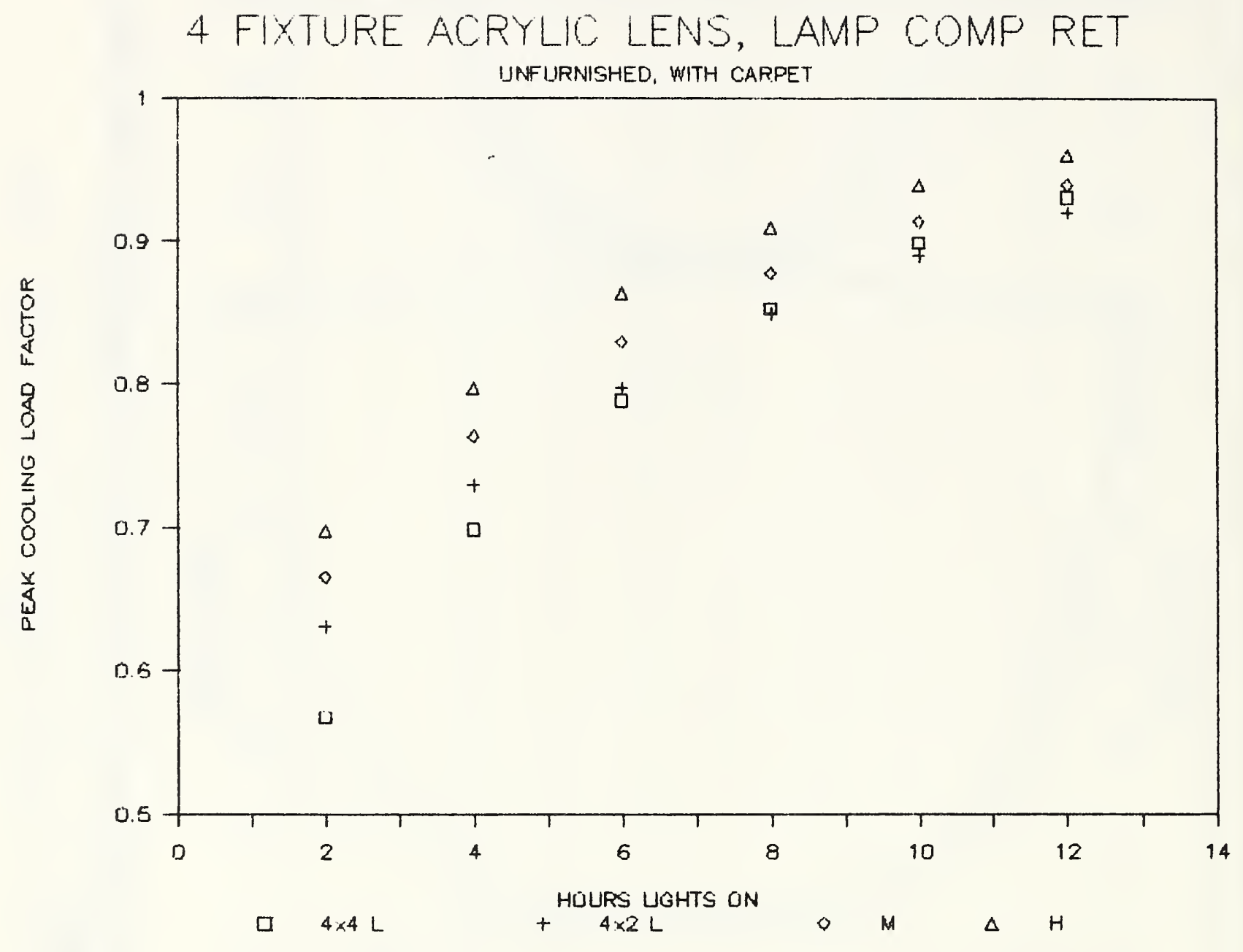

Figure 74. Peak cooling load factor for four fixture acrylic lens luminaires with lamp compartment return, carpet 


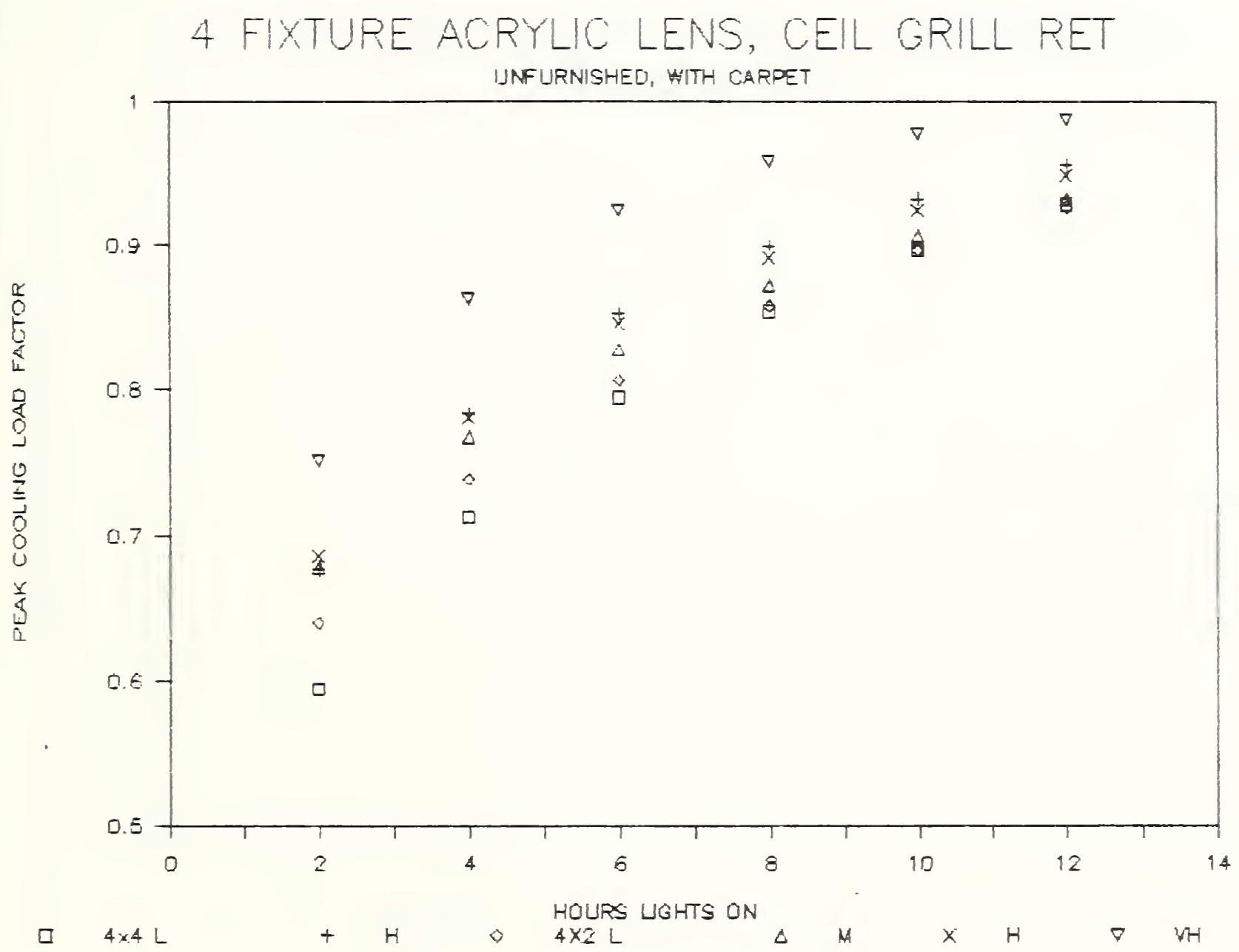

Figure 75. Peak cooling load factor for four fixture acrylic lens luminaires with ceiling grill retum, carpet 


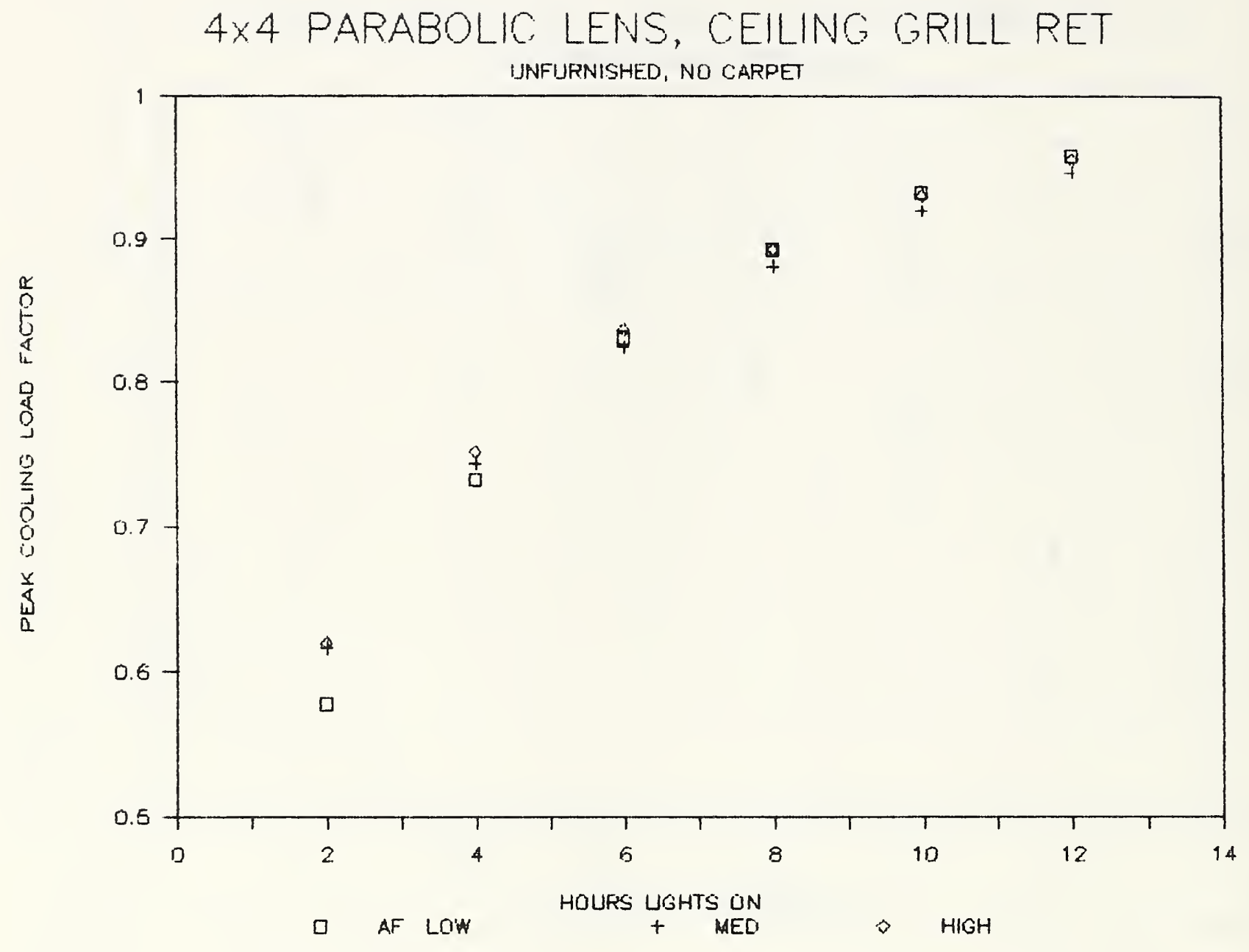

Figure 76. Peak cooling load factor for four four-lamp parabolic diffuser luminaires with ceiling grill return 


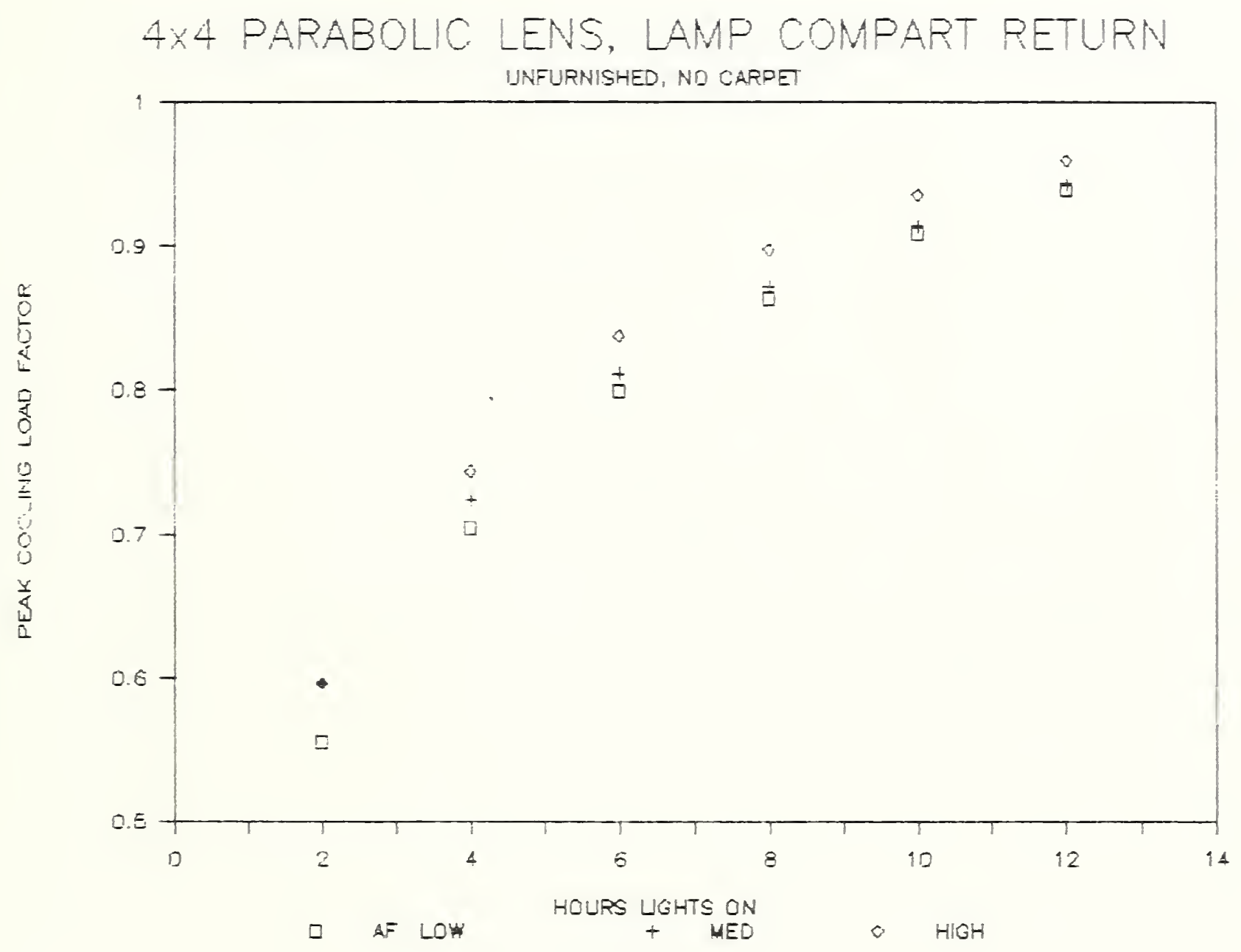

Figure 77. Peak cooling load factor for four four-lamp parabolic diffuser luminaires with lamp compartment retum 


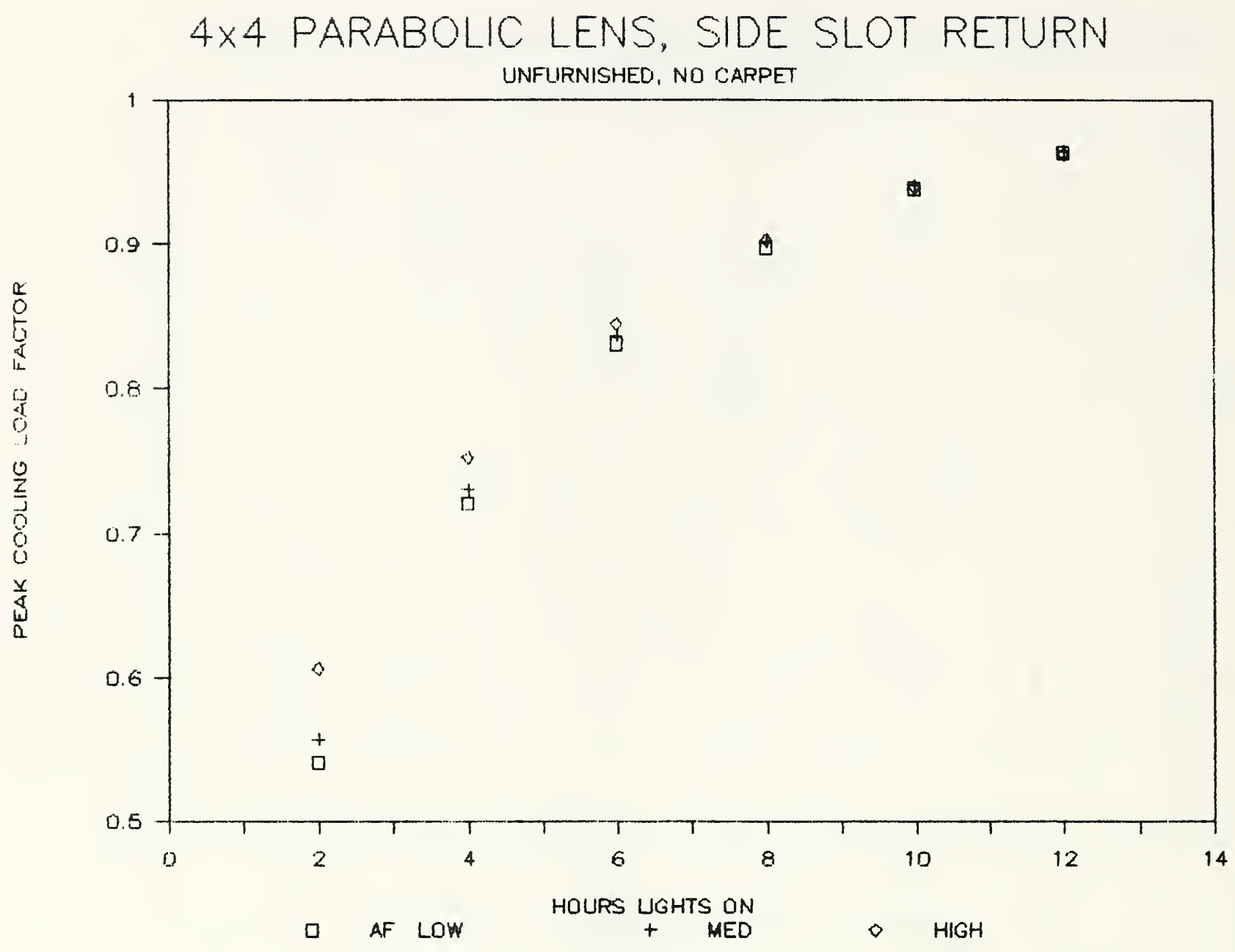

Figure 78. Peak cooling load factor for four four-lamp parabolic diffuser luminaires with side slot return 


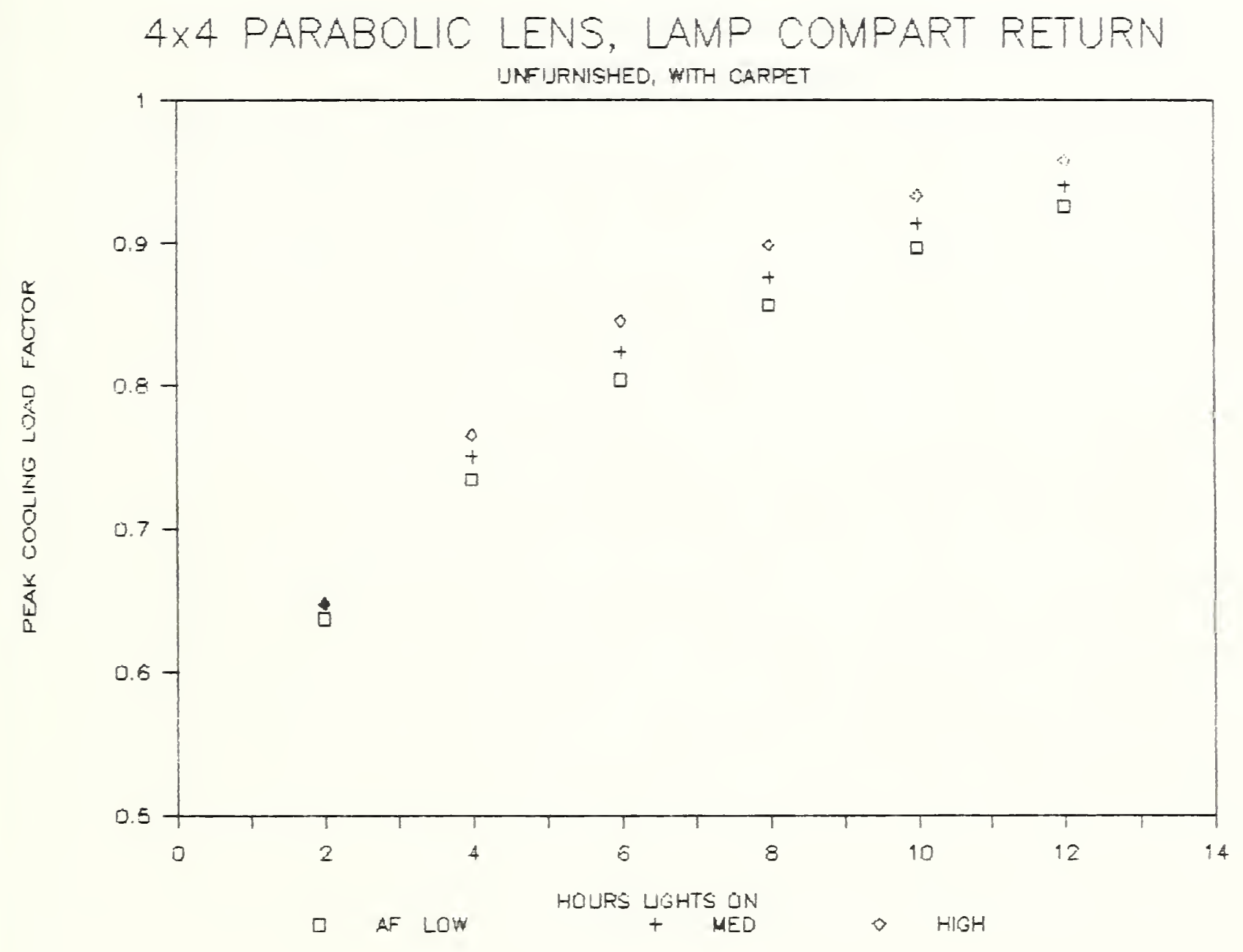

Figure 79. Peak cooling load factor for four four-lamp parabolic diffuser luminaires with lamp compartment return, carpet 


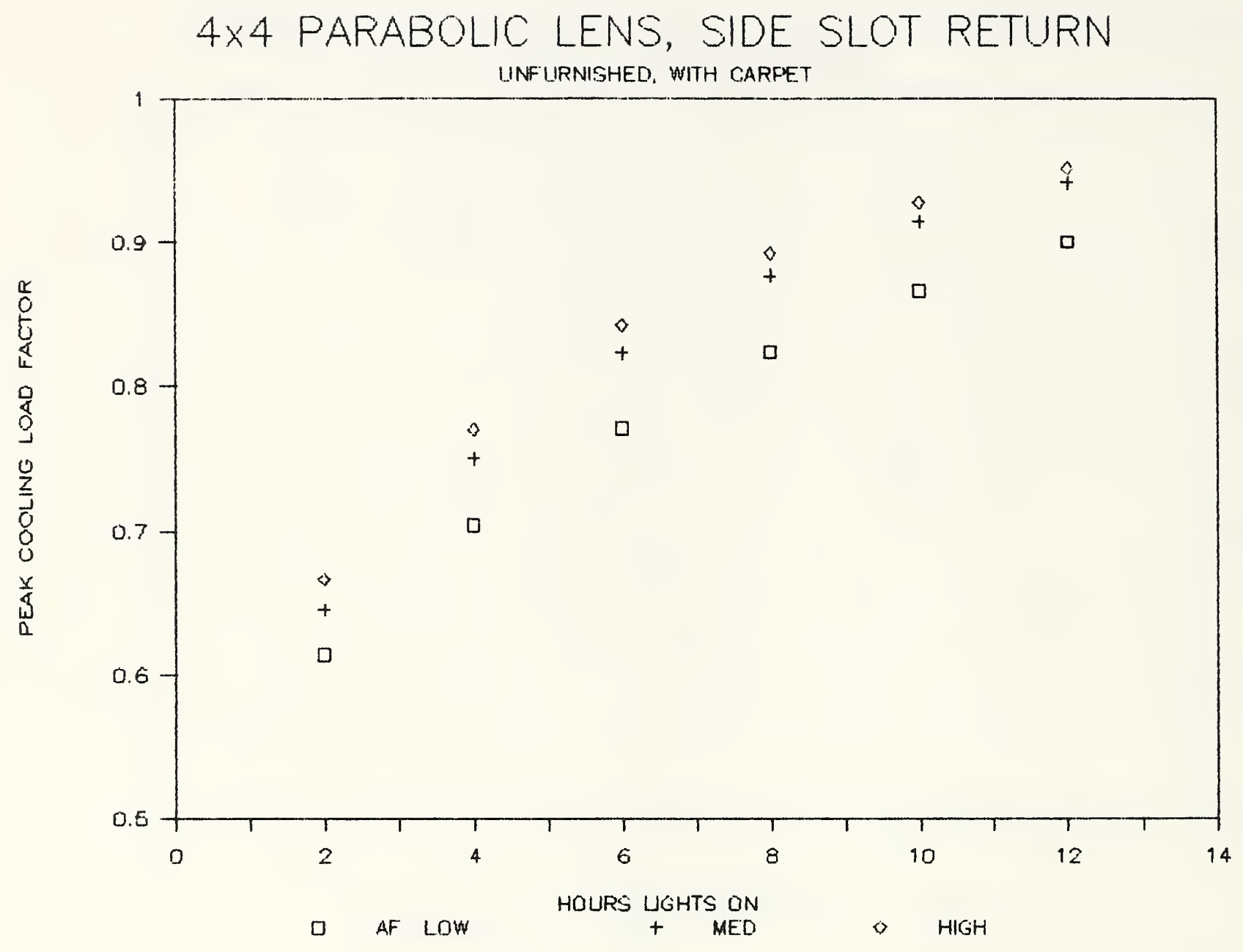

Figure 80. Peak cooling load factor for four four-lamp parabolic diffuser luminaires with side slot return, carpet 


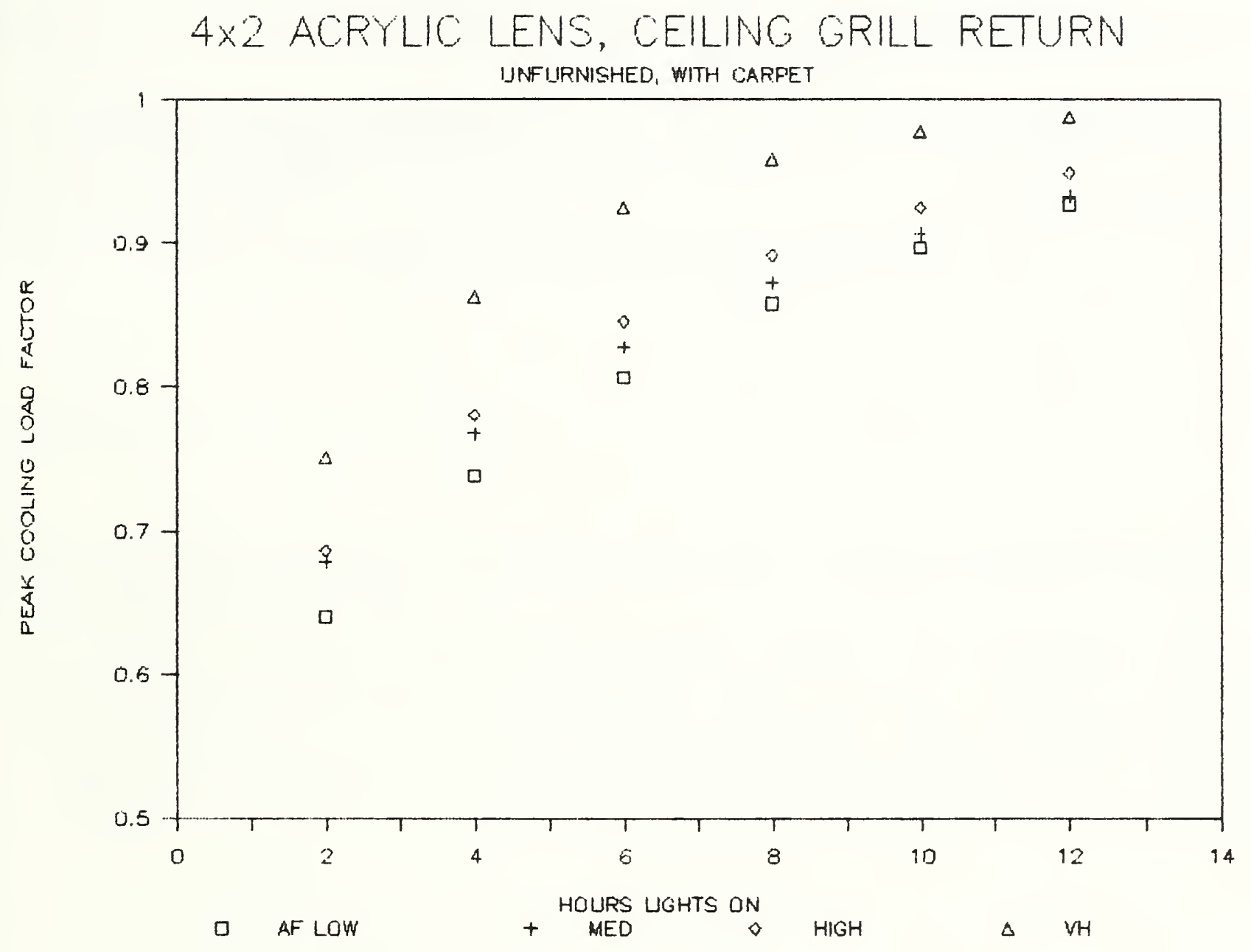

Figure 81. Peak cooling load factor for four two-lamp acrylic lens luminaires with ceiling grill return, carpet 


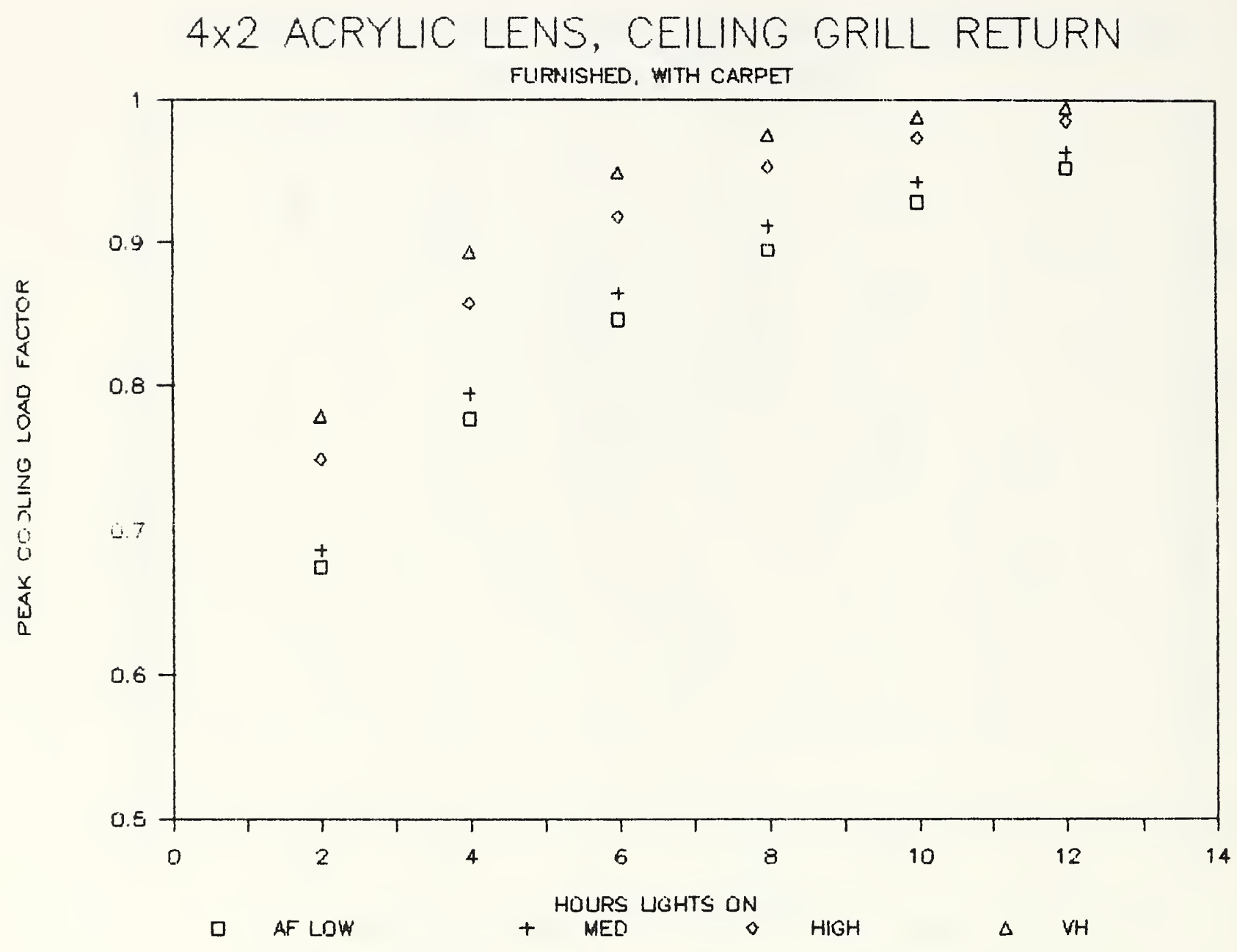

Figure 82. Peak cooling load factor for four two-lamp acrylic lens luminaires with ceiling grill return, carpet, furnished 


\section{BIBLIOGRAPHIC DATA SHEET}

2. PERFORMING ORGANIZATION REPORT NUMBER

3. PUBLCATION DATE

MARCH 1992

4. TITLE AND SUBTITLE

The Interaction of Lighting, Heating and Cooling Systems in Buildings

5. AUTHOR(S)

Stephen J. Treado, John W. Bean

6. PERFORMING ORGANIZATION (IF JOINT OR OTHER THAM NIST, SEE INSTRUCTIONS)

U.S. DEPARTMENT OF COMMERCE

MATIONAL INSTITUTE OF STANDARDS AND TECHNOLOGY

GAITHERSBURG, MD 20899

7. CONTRACT/GRANT NUMBER

8. TYPE OF REPORT AND PERIOD COVERED

9. SPONSORING ORGAMIZATION NAME AND COMPLETE ADDRESS (STREET, CITY, STATE, ZIP)

10. SUPPLEMENTARY NOTES

11. ABSTRACT (A 200-WORD OR LESS FACTUAL SUMMARY OF MOST SIGNIFICANT INFORMATION. IF DOCUMENT INCLUDES A SIGNIFICANT BIBLOGRAPHY OR UTERATURE SURVEY, MENTION IT HERE.)

The interaction of building lighting and HVAC systems, and the effects on cooling load and lighting system performance, are being evaluated using a full-scale test facility at the National Institute of Standards and Technology. The results from a number of test configurations are described, including lighting system efficiency and cooling load due to lighting. The effect of lighting and HVAC system design and operation on performance is evaluated. Design considerations are discussed.

12. KEY WORDS (6 TO 12 ENTRIES; ALPHABETICAL ORDER; CAPTTALZE ONLY PROPER MAMES; AND SEPARATE KEY WORDS BY SEMICOLONS) airflow, cooling, energy, lighting, load calculation, ventilation

FOR OFFICLAL DISTRIBUTION. DO NOT RELEASE TO MATIONAL TECHNICAL INFORMATION SERVICE (NTIS).

ORDER FROM SUPERINTENDENT OF DOCUMENTS, U.S. GOVERNMENT PRINTING OFFICE, WASHINGTON, DC 20402

ORDER FROM NATIONAL TECHNICAL INFORMATION SERVCE (NTIS), SPRINGFELD, VA 22161.

14. HUMBER OF PRINTED PAGES

\section{5}

15. PRICE

A06 


